United States Department of Commerce Technology Administration

National Institute of Standards and Technology

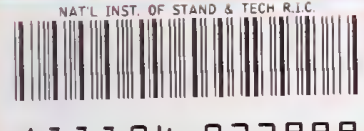

NIST

PUBLICATIONS

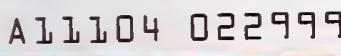

NIST Special Publication 791, 1993 Edition

State Weights and Measures Laboratories: State Standards Program Description and Directory

Georgia L. Harris

QC
100
.457
非791
1993


$T$ he National Institute of Standards and Technology was established in 1988 by Congress to "assist industry in the development of technology ... needed to improve product quality, to modernize manufacturing processes, to ensure product reliability . . . and to facilitate rapid commercialization ... of products based on new scientific discoveries."

NIST, originally founded as the National Bureau of Standards in 1901, works to strengthen U.S. industry's competitiveness; advance science and engineering; and improve public health, safety, and the environment. One of the agency's basic functions is to develop, maintain, and retain custody of the national standards of measurement, and provide the means and methods for comparing standards used in science, engineering, manufacturing, commerce, industry, and education with the standards adopted or recognized by the Federal Government.

As an agency of the U.S. Commerce Department's Technology Administration, NIST conducts basic and applied research in the physical sciences and engineering and performs related services. The Institute does generic and precompetitive work on new and advanced technologies. NIST's research facilities are located at Gaithersburg, MD 20899, and at Boulder, CO 80303. Major technical operating units and their principal activities are listed below. For more information contact the Public Inquiries Desk, 301-975-3058.

Technology Services

- Manufacturing Technology Centers Program

- Standards Services

- Technology Commercialization

- Measurement Services

- Technology Evaluation and Assessment

- Information Services

\section{Electronics and Electrical Engineering} Laboratory

- Microelectronics

- Law Enforcement Standards

- Electricity

- Semiconductor Electronics

- Electromagnetic Fields ${ }^{1}$

- Electromagnetic Technology ${ }^{1}$

\section{Chemical Science and Technology}

Laboratory

- Biotechnology

- Chemical Engineering ${ }^{1}$

- Chemical Kinetics and Thermodynamics

- Inorganic Analytical Research

- Organic Analytical Research

- Process Measurements

- Surface and Microanalysis Science

- Thermophysics ${ }^{2}$

Physics Laboratory

- Electron and Optical Physics

- Atomic Physics

- Molecular Physics

- Radiometric Physics

- Quantum Metrology

- Ionizing Radiation

- Time and Frequency ${ }^{1}$

- Quantum Physics ${ }^{1}$
Manufacturing Engineering Laboratory

- Precision Engineering

- Automated Production Technology

- Robot Systems

- Factory Automation

- Fabrication Technology

Materials Science and Engineering Laboratory

- Intelligent Processing of Materials

- Ceramics

- Materials Reliability

- Polymers

- Metallurgy

- Reactor Radiation

Building and Fire Research Laboratory

- Structures

- Building Materials

- Building Environment

- Fire Science and Engineering

- Fire Measurement and Research

Computer Systems Laboratory

- Information Systems Engineering

- Systems and Software Technology

- Computer Security

- Systems and Network Architecture

- Advanced Systems

\section{Computing and Applied Mathematics}

Laboratory

- Applied and Computational Mathematics ${ }^{2}$

- Statistical Engineering ${ }^{2}$

- Scientific Computing Environments ${ }^{2}$

- Computer Services ${ }^{2}$

- Computer Systems and Communications ${ }^{2}$

- Information Systems

At Boulder, CO 80303.

${ }^{2}$ Some elements at Boulder, CO 80303. 


\section{NIST Special Publication 791, 1993 Edition}

\section{State Weights and Measures Laboratories: State Standards Program Description and Directory}

Georgia L. Harris

Office of Weights and Measures

National Institute of Standards and Technology

Gaithersburg, MD 20899

(Supersedes NIST Special Publication 791, 1992 Edition)

June 1993

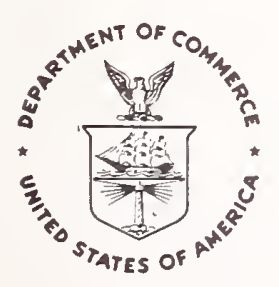

U.S. Department of Commerce

Ronald H. Brown, Secretary

National Institute of Standards and Technology

Arati Prabhakar, Director 
National Institute of Standards and Technology

Special Publication 791, 1993 Ed. (Supersedes SP 791, 1992 Ed.)

Natl. Inst. Stand. Technol.

Spec. Publ. 791, 1993 Ed.

130 pages (June 1993)

CODEN: NSPUE2
U.S. Government Printing Office Washington: 1993
For sale by the Superintendent of Documents U.S. Government Printing Office Washington, DC 20402 


\section{Foreword}

In support of its mission to promote uniform standards of measurement throughout the country, the National Institute of Standards and Technology (NIST) received funding from Congress in 1965 to provide new standards of mass, length, and volume and the equipment needed to perform calibrations in these measurement areas to State weights and measures laboratories. This program, called the [New] State Standards Program was established at NIST; to maintain the validity of these standards and to assure that the standards are used properly, NIST also:

(1) trains State metrologists in the test procedures used in the NIST laboratories; and

(2) conducts a voluntary accreditation program for State weights and measures laboratories.

Part I of this publication describes the accreditation activities of the State Standards Program. There are three general requirements for accreditation:

(1) the State must have adequate facilities, equipment, and standards to perform the measurements;

(2) the State metrologist must receive the necessary training and submit data demonstrating the results of applying test procedures; and

(3) the laboratory must establish measurement control programs acceptable to NIST in the various measurement areas.

This approach to laboratory accreditation was endorsed by the 1983 National Conference on Weights and Measures. The Office of Weights and Measures at NIST has proposed the addition of accreditation programs for State laboratories in the areas of Grain Moisture and Petroleum Quality Testing since they have been requested by the State Weights and Measures agencies. Some tentative guidelines are outlined in Part I; specific accreditation requirements will be published in NIST Handbook 143, Program Handbook, or other appropriate document.

Part II is the directory of State weights and measures laboratories and lists the services they provide to State and local weights and measures agencies as well as to industry. The directory is intended to assist potential users of the laboratory services in locating and obtaining needed measurement services. The directory provides a summary table of measurement services and programs as well as the following information for each State or accredited laboratory:

(1) the accreditation period, if accredited by NIST;

(2) the laboratory staff member(s), address, and phone number;

(3) services available; and

(4) fees, if any, for services.

One objective of the Omnibus Trade and Competitiveness Act of 1988 is to make the International System (SI) of Units the primary measurement system in the United States. SI Units are the preferred measurement system in the State laboratories. State laboratories have primary standards in both "customary" and SI units; the laboratory equipment employs SI units; and training for State metrologists primarily uses the SI System of Units. However, commercial equipment for sales to the public for the most part entails "customary" units. Descriptions of measurement services in this directory refer to whichever system typically predominates for the type of service provided, although capabilities exist for measurement services in both systems of units. 
Note regarding ISO 9000:

During 1992, the Office of Weights and Measures, with cooperation from the National Voluntary Laboratory Accreditation Program (NVLAP), began the process of revising the State Laboratory Accreditation Program to include the adoption of ISO Guide 25. Accreditation requirements are currently being revised, but were not completely in place as of the publication date of this directory. However, many of the quality criteria identified in ISO Guide 25 are already in place in the State laboratories. Technical criteria following ISO Guide 25 are being developed; they will be consistent with international recommendations. Many State laboratories have begun the process of implementing the new requirements on a voluntary basis, including revision of their current laboratory Quality Manuals to meet ISO Guide 25 requirements and continuation of a formal annual self-assessment, with review by the Office of Weights and Measures. All laboratories accredited by OWM can provide accurate and traceable measurement results; competence is regularly demonstrated through round robin measurements. The Office of Weights and Measures believes that once laboratories have been accredited under the new criteria, they will fully meet ISO 9000 service requirements as well as registration requirements for ISO 9002. Most NIST/OWM accredited laboratories will meet customer requirements for ISO 9000 now. 


\section{Contents}

Foreword $\ldots \ldots \ldots \ldots \ldots \ldots \ldots \ldots \ldots \ldots \ldots \ldots \ldots \ldots \ldots \ldots \ldots \ldots \ldots \ldots$

PART I - Program Description $\ldots \ldots \ldots \ldots \ldots \ldots \ldots \ldots \ldots \ldots \ldots \ldots \ldots \ldots \ldots$

Introduction $\ldots \ldots \ldots \ldots \ldots \ldots \ldots \ldots \ldots \ldots \ldots \ldots \ldots \ldots \ldots \ldots \ldots \ldots$

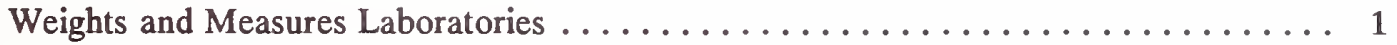

National Type Evaluation Program ........................ 1

Accredited Measurement Areas $\ldots \ldots \ldots \ldots \ldots \ldots \ldots \ldots \ldots \ldots \ldots \ldots \ldots \ldots$

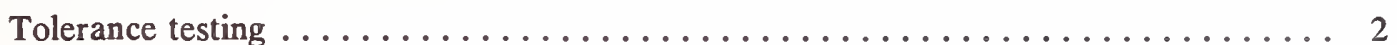

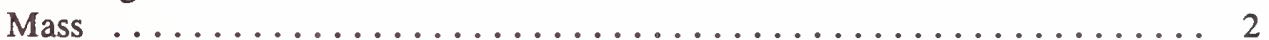

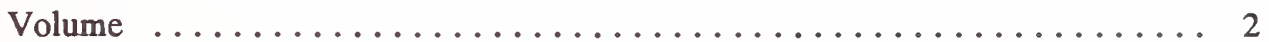

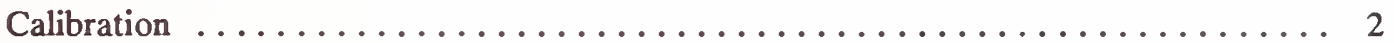

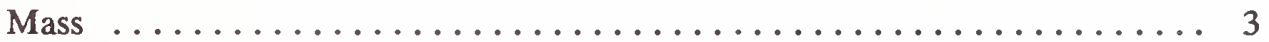

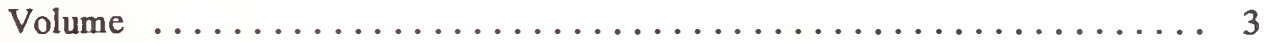

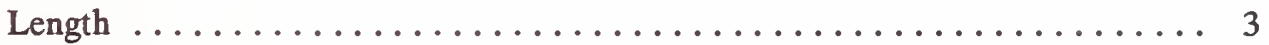

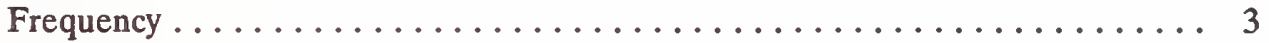

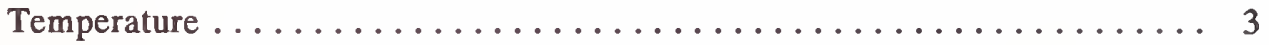

Accreditation Requirements $\ldots \ldots \ldots \ldots \ldots \ldots \ldots \ldots \ldots \ldots \ldots \ldots \ldots \ldots$

Weights and Measures Laboratory Requirements ................. 3

Training and Evaluation ........................... 4

Measurement Control ....................... 5

National Type Evaluation Program $\ldots \ldots \ldots \ldots \ldots \ldots \ldots \ldots \ldots \ldots$

National Type Evaluation Program Requirements ................. 6

Unevaluated Measurement Areas . . . . . . . . . . . . . . . . . . 7

Proposed Grain Moisture Laboratory Program and Accreditation Requirements ..... 7

Proposed Petroleum Quality Laboratory Program and Accreditation Requirements ... 8

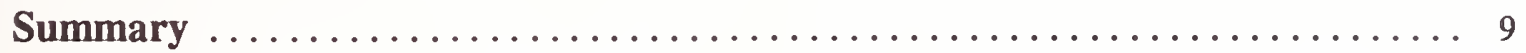

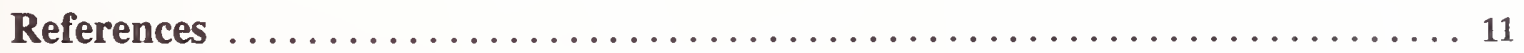

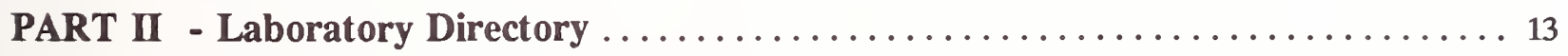

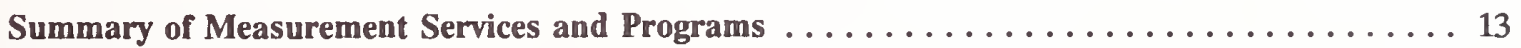

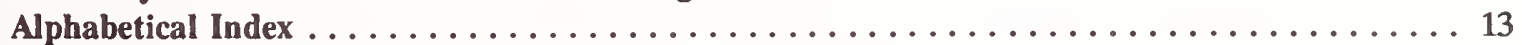





\section{PART I - Program Description}

\section{Introduction}

\section{Weights and Measures Laboratories}

In 1965, Congress funded the National Institute of Standards and Technology (NIST, formerly the National Bureau of Standards) to establish the [New] State Standards Program to provide new standards of mass, volume, and length to the States, the District of Columbia, Puerto Rico, and the Virgin Islands to update their weights and measures laboratories and increase their measurement capabilities. The program also provided the laboratory equipment necessary for the States to use the standards in their measurement services.

New standards and equipment were issued to the States from 1967 through 1978. Since that time, many States have purchased additional equipment to increase their measurement capability and to expand into other measurement areas, such as liquid-inglass thermometry, the frequency testing of tuning forks for police radar guns, and the testing of large volume and liquified petroleum gas (LPG) provers.

Prior to receipt of the standards and equipment, each jurisdiction was required to provide a laboratory facility meeting specifications established under the State Standards Program. In addition, the laboratory metrologist was required to complete training at NIST in the use of the standards and the equipment.

Subsequent to the issue of standards and equipment to the States, NIST instituted a program whereby State weights and measures laboratories are accredited by measurement areas. Accreditation of
State weights and measures laboratories is a part of the current State Standards Program. NIST does not certify the measurements made by a State, but formally recognizes that the State laboratory has an apparent capability to perform reliable measurements and that the metrologist has been trained in the proper procedures to provide these measurements. The accreditation also indicates that the metrologist has fulfilled the requirements and submitted the data requested by NIST for accreditation. Each State laboratory is responsible for verifying its measurement traceability.

A laboratory that has not been accredited does not necessarily mean that it is inadequate or that its measurements are not valid. Rather, it indicates that a laboratory has not submitted the information necessary to permit NIST to evaluate its capability. Consequently, NIST cannot take a position regarding the capability of a nonaccredited laboratory.

\section{National Type Evaluation Program}

Under the National Type Evaluation Program (NTEP), new weighing and measuring devices are tested and evaluated to determine if they comply with the requirements in NIST Handbook 44, "Specifications, Tolerances, and Other Technical Requirements for Weighing and Measuring Devices" [1] (see references p. 11). The Program is administered by NIST in support of the National Conference on Weights and Measures. Tested devices are intended for use in the commercial 
measurement system, that is, for use in weighing or measuring commodities to be sold to the general public.

Several State laboratories and the Federal Grain Inspection Service (FGIS) have acquired the capability, and have been authorized by NIST, to conduct type evaluations under the NTEP. These laboratories, and the devices that they are authorized to evaluate, are identified in this publication.

\section{Accredited Measurement Areas}

A brief summary of each measurement area in the accreditation program is given under the headings of Tolerance Testing and Calibration. Some States offer services in other measurement areas (see Unevaluated Measurement Areas in Part I, and directory listings in Part II) but, since NIST has not yet developed criteria to serve as the basis for laboratory accreditation in these areas, they are not included in the current accreditation program.

Each laboratory accredited in a given area has been judged to be capable of providing the measurement services indicated. The range of standards that can be tested by a laboratory will depend upon the equipment it has purchased in addition to that received through the State Standards Program.

A general classification of the range of standards that can be tested is indicated by the headings in the tables of services available (see Part II). The mass classifications for tolerance testing are given in pounds because inch-pound standards are usually used. Mass classifications for calibrations are given in kilograms because metric standards are used. Comparable standards in other mass units of measure can also be tested.

\section{Tolerance testing}

A tolerance may be simply defined as the permitted difference between the nominal value of the standard and its actual value under specific conditions. Tolerance testing is the process for determining if the actual value of a standard is within tolerance. If a standard is not within tolerance it may be adjusted to bring the value within tolerance. To be accredited to a tolerance class, a standard must comply with the specifications and tolerances for the specified tolerance class.

Mass

Tolerance testing of mass standards is usually done according to specifications included in:

- $\quad$ NIST Handbook 105-1 [2];

- $\quad$ American Society for Testing and Materials (ASTM) Standard E 617-91, Classes 4, 5, and 6 [3];

- International Organization of Legal Metrology, Classes $\mathrm{M}_{1}, \mathrm{M}_{2}, \mathrm{M}_{3}$ [6].

Laboratories may also test to NBS Circular 547, Classes P, Q, and T [4], or to NBS Circular 3, Classes A, B, and C [5]; however, these classes have been superseded by ASTM weight classifications.

Volume

Tolerance testing of volume standards is divided into small-, intermediate-, and large-volume standards, depending upon the laboratory standards to be used. A small-volume standard may have a capacity up to 25 gallons. The capacities of intermediate-volume standards range from 25 through 500 gallons. Large-volume standards have capacities in excess of 500 gallons. Though metric units (liters) are occasionally tested, the principal standard of volume tested is defined in units of gallons.

Glassware standards may be tested to NBS Handbook 105-2 [7] specifications and tolerances or to the tolerances stated in Federal regulations. Metal field standards are tested to NBS Handbook 105-3 [8] specifications and tolerances.

\section{Calibration}

Calibration is the process of comparing an unknown standard to a known standard and assigning a value, along with an uncertainty, to the unknown standard. The uncertainty expresses the extent to which that assigned value may be in error relative to its reference base, which typically is the national standard. 
When calibrated by a State laboratory, the assigned value may or may not be within the specified tolerance. The correction and uncertainty assigned to the standard should therefore be used in subsequent measurements. In some cases, the assigned uncertainties will exceed the specified tolerances. The uncertainties, which reflect the level of precision the State laboratories can achieve, will differ from one laboratory to another depending on standards, the competence of the metrologist, and equipment available to perform the measurement. Potential customers should contact the State weights and measures laboratory to verify that the assigned uncertainty it typically provides is adequate for their needs.

Mass

Calibrations are usually performed on mass standards designed to meet specifications and tolerances in:

- $\quad$ American Society for Testing and Materials (ASTM) Standard E 617-91, Classes 1, 1.1, 2 , and 3;

\section{International Organization of Legal} Metrology Classes $\mathrm{E}_{2}$ and $\mathrm{F}_{1}$.

Laboratories may also test to NBS Circular 547, Classes J, M, S, and S-1; however, these classes have been superseded by ASTM weight classifications.

Depending on the laboratory, air buoyancy corrections may or may not be made. Customers should specify the apparent mass reference density desired (usually apparent mass versus $8.0 \mathrm{~g} / \mathrm{cm}^{3}$ ) for their calibration and provide the density of their mass standards.

\section{Volume}

Small glassware standards are usually calibrated gravimetrically or by volume transfer. The capacity of metal standards is typically 20 liters or 5 gallons. Metal provers larger than 5 gallons may be calibrated if appropriate standards are available.

\section{Length}

Steel tapes up to several hundred feet long may be calibrated. The uncertainties are typically a few thousandths of a foot. Rigid rules up to 12 inches long can be calibrated with uncertainties of several thousandths of an inch. Most requests for length tests have not been in metric units, though the states are capable of making these measurements.

\section{Frequency}

The frequency calibration services are usually limited to tuning forks used with police radar guns.

\section{Temperature}

Temperature calibration is generally limited to liquid-in-glass thermometers. Some States test only clinical thermometers of this type. The standards for most States are also liquid-in-glass thermometers, though some use platinum resistance thermometer standards. The uncertainties for temperature calibration will vary among laboratories. Many requests for calibrations involve tolerances specified by the customer.

\section{Accreditation Requirements}

\section{Weights and Measures Laboratory Requirements}

There are three general requirements for accreditation of State weights and measures laboratories:

(1) The State must have adequate laboratory facilities, equipment, and standards to provide the services that are offered.

The State metrologist must:

(a) successfully complete the NIST Basic Metrology Seminar; (b) maintain competency through active (annual attendance at meetings and participation in round robin experiments) participation in the Regional Measurement Management Programs (RMMPs); and (c) demonstrate the ability to perform the laboratory measurements, which may be done through 
the RMMPs, NIST Intermediate Metrology

Seminar, or special assignments.

The metrologist must implement and maintain measurement control programs in various measurement areas as required by NIST.

The requirements for the laboratory facilities and general operation are contained in NBS Handbook 143, "State Weights and Measures Laboratories Program Handbook" [9]. Each laboratory is evaluated using a self-appraisal checklist (contained in Handbook 143), submitted by the metrologist, and subject to verification by an on-site evaluation.

The metrologist is evaluated on the basis of performance in training seminars, the work submitted to NIST as part of laboratory assignments, participation in technical meetings, and through review of laboratory data and test procedures during the on-site evaluation. The quality of the laboratory's measurement results are evaluated through its control charts and round robin experiments. Brief discussions of these topics follow.

\section{Training and Evaluation}

A suitable technical degree, such as Engineering, Physics, Mathematics, or Chemistry is highly recommended. Working knowledge of algebra and basic statistics is essential. It is expected that metrologists will have spent time in the laboratory familiarizing themselves with the equipment prior to attendance at a Basic Laboratory Seminar.

\section{Basic Laboratory Training}

NIST trains State metrologists in the basic laboratory metrology procedures for mass, volume, and length measurement during a 2-week Basic Laboratory Metrology seminar. The test procedures are described in NIST publications, including NBS Handbook 145, "Handbook for the Quality Assurance of Metrological Measurements" [10]. The metrologists must then complete a series of laboratory assignments and submit the data to NIST to document their understanding of the procedures and the calculations. Additional laboratory problems are assigned to each laboratory to establish measurement control programs in several measurement areas.

\section{Intermediate and Advanced Laboratory Training}

Each metrologist is expected to attend the Intermediate Metrology Seminar within 2 years of attendance at the Basic Seminar to receive additional training in laboratory procedures and in the corrections needed for high precision measurement. Following this seminar, metrologists are assigned a series of problems so that they can demonstrate their understanding of the seminar material.

States that expand into other areas of measurement are encouraged to attend specialized seminars conducted by NIST calibration laboratories. Examples of these areas are temperature and pressure measurements. The laboratories must then establish measurement control programs in these measurement areas to ensure agreement with NIST results.

\section{Regional and National Programs}

At least one metrologist from each State must participate in Regional Measurement Management Programs (RMMPs). These RMMPs are groups of laboratories in given geographical areas that have joined together to: (1) conduct meetings for the purpose of training and demonstration of procedures; and (2) to conduct round robin experiments to promote uniform measurements in their geographical area. The round robin experiments provide an essential link in the measurement traceability between NIST and the States. The knowledge exhibited by metrologists in these meetings and their results on the round robin experiments are included as part of the evaluation of the State laboratories.

In addition to training seminars and regional metrology groups, the Metrology Workshop at the annual meeting of the National Conference on Weights and Measures (NCWM) coordinates the efforts of the regional groups and provides the opportunity for metrologists to become active in weights and measures metrology issues of a national and international nature. The knowledge and participation demonstrated by the metrologists at the annual workshops as well as participation 
throughout the year in NCWM/NIST work projects afford metrologists the opportunity to provide technical input to the NCWM. This activity not only enhances the NCWM program and the metrologist's skills and abilities, but allows further evaluation of metrologists' special capabilities.

\section{Measurement Control}

NIST criteria require State weights and measures laboratories to utilize formal quality control techniques to ensure that their measurements are sufficiently accurate to meet customer needs on a continuing basis. If these techniques are properly employed, it is possible to quantify the uncertainties of the measurements. Monitoring uncertainties over time can assure that they remain sufficiently small to meet operational requirements. NIST has been working closely with the State weights and measures laboratories to help enhance laboratory programs and to demonstrate their ability to perform highquality calibrations.

Measurement control programs have been established for mass calibration, the volume transfer testing of glass volume standards, and a number of other measurement areas, such as tolerance testing and 5-gallon gravimetric control charts. Round robin experiments are conducted to investigate the agreement of the State laboratories in all accredited measurement areas.

The measurement control programs consist of two parts: (1) internal control programs; and (2) external control programs.

\section{Internal Control Programs}

The internal measurement control programs generally consist of repeated measurements on the same objects over an extended period of time. The data are then plotted on control charts to establish the limits of random errors in the measurement processes. Repeated measurements made on the standards themselves monitor the standards as well as the variability of the measurement process. Statistical confidence tests monitor the stability of the standards and the variance of the measurement process. The control chart data can be used to document the validity of the measurements made on a given day. Finally, the control chart data are used to determine if the standards agree with NIST- reported values. Disagreement with NIST-reported values requires corrective action. If a problem persists, the standards must be recalibrated at NIST.

In some measurement areas it is not possible to keep a standard or standards in the laboratory simply for the purpose of measurement control. For example, it is impractical to keep an extra 100gallon prover in the laboratory to run as a check standard during every 100-gallon prover test. In these instances, repeated measurements are run on each standard submitted for test. Differences in test results are then plotted on a "range" chart. The process variance is estimated from the range in the measurements made on many standards. The range chart is also used to verify that repeated measurements made on a given standard fall within the indicated limits.

In some instanices, such as the volume transfer testing of glass flasks, a combination of control and range charts is used. The type of measurement control program depends on the criticality of the measurements, the availability of standards to use as "check" standard, and the tolerances for the standards under test. The frequency of measurements made to control the measurement process and the type of control program utilized are tailored to the measurement area under investigation.

\section{External Control Programs}

The NIST goal is to provide an external measurement control system that is available to all States. The overall goal is for each State to actively participate in one of the five formal Regional Measurement Management Programs (RMMPs). The RMMPs promote uniform measurement results by:

(1) providing training through the presentation of papers prepared by members;

(2) demonstrating test procedures to assure uniformity;

(3) conducting round robin experiments to investigate the presence of measurement errors; and

addressing mutual measurement concerns.

The RMMPs are groups of State and, to a limited extent, private laboratories that have joined together 
to conduct meetings and perform round robin tests in mass, length, volume, and to a limited extent other measurement areas such as grain moisture, thermometry, and tuning fork testing, to promote uniformity in test procedures and measurement results. The five RMMPs listed below provide the opportunity for all States, the District of Columbia, Puerto Rico, and the Virgin Islands to participate. A map of the regional groups is shown in figure 1 .

$$
\begin{aligned}
& \text { Northeastern Measurement Assurance } \\
& \text { Program (NEMAP) } \\
& \text { Southeastern Measurement Assurance } \\
& \text { Program (SEMAP) } \\
& \text { Western Regional Assurance Program } \\
& \text { (WRAP) } \\
& \text { Mid-America Measurement Assurance } \\
& \text { Program (MidMAP) } \\
& \text { Southwestern Assurance Program (SWAP) }
\end{aligned}
$$

In the external measurement control programs, standards external to the laboratory are submitted to the laboratory for test. These external standards are normally calibrated by NIST (or FGIS in the case of grain moisture) to provide a reference value. These standards are usually circulated as part of round robin experiments conducted and coordinated by the RMMPs. Although NIST provides values for the circulating standards, the round robin experiments are developed, coordinated, and analyzed by the RMMPs. NIST participates in the data analysis and the regional meetings to identify any problems and to provide training to eliminate discrepancies. This information is vital to the NIST accreditation program. The RMMPs are thus an integral part of the NIST accreditation program and reduce the amount of NIST effort necessary to monitor State laboratories.

The external measurement control programs are used to compare the RMMP results to NIST or other national standard values. These programs investigate the presence of systematic errors and can be used to test the variance of the measurement processes. Plots of interlaboratory data results are frequently used to analyze data. To obtain maximum benefits from these external measurement control programs, internal measurement control programs should be established before a laboratory participates in round robin experiments.
Uniformity among RMMPs is attained through additional NIST training at the meetings, the calibration of standards by NIST used in round robin experiments, and the evaluation of the round robin results. The RMMPs interact further through the Metrology Workshop held in conjunction with the Annual Meeting of the National Conference on Weights and Measures. Members of each RMMP report activities and round robin results and discuss ways of improving the quality of the laboratories as well as individual proficiency.

A sixth regional measurement group was developed in the Caribbean area during 1992. Participants in the Caribbean Measurement Assurance Program (CaMAP) group include a number of countries in the Caribbean region. Since these laboratories are participating in the regional group on a voluntary basis, they have not formed a formal relationship, and they are outside the scope of the responsibility for the Office of Weights and Measures, they have not been evaluated according to accreditation criteria.

Participation in the regional measurement group meetings or in the round robin measurements has included such countries as Canada, Jamaica, Grenada, Trinidad \& Tobago, St. Lucia, Mexico, Ecuador, and the Dominican Republic.

\section{National Type Evaluation Program}

NIST will authorize qualified State and other laboratories to: (1) conduct complete testing and evaluation; or (2) conduct field performance testing of specified types of devices under the NTEP. Type evaluations are primarily conducted on scales, weighing systems, meters, metering systems, and devices associated with these applications. The specific types of devices that a Participating Laboratory is authorized to evaluate are identified in the directory.

\section{National Type Evaluation Program Requirements}

The objectives of the authorization plan of the National Type Evaluation Program are to:

promulgate uniform criteria and procedures for testing laboratories; 
authorize those qualifying as "Participating Laboratories" in the Program; assure the uniform application of NTEP device evaluation criteria; and provide the basis for acceptance by the States and Federal agencies of the evaluations conducted in the authorized Participating Laboratories.

To be authorized, laboratories must demonstrate that they have the staff, equipment, and resources to conduct the evaluations. The ability to conduct type evaluations is heavily dependent on the people who do the evaluations. They must have knowledge of Handbook 44 requirements, device applications, and test procedures. They must also have the analytical skills and judgment to determine whether the requirements are satisfied.

Because the nature of type evaluations is very different from laboratory metrology, staff other than the laboratory metrologist are often authorized to conduct type evaluations for NTEP.

Type evaluations may include both laboratory and field tests. A State must have the specialized equipment required to test a device over a temperature range (i.e., an environmental chamber along with other specialized equipment). Field evaluations require much of the same equipment used in weights and measures enforcement testing, but the test procedures are much more extensive. NIST provides extensive training on the NTEP criteria, test procedures, and the rationale behind the requirements. The laboratory staff must demonstrate a thorough knowledge of the criteria and test procedures, the ability to properly analyze the test data and device performance as it pertains to the device applications, and demonstrate the ability to make judgments consistent with NTEP policy. Regular communication with NIST regarding new device features and evaluation criteria is required to maintain uniformity among the NTEP laboratories.

\section{Unevaluated Measurement Areas}

In addition to the accredited measurement areas of tolerance testing, calibration, and device type evaluations, many laboratories perform measurements for which the NIST Office of
Weights and Measures has not established guidelines for accreditation. These measurement areas can include the testing of dial gauges used to test polyethylene sheeting (an extension of dimensional measurements) or entire programs such as grain moisture testing and petroleum quality testing. This publication contains new information regarding proposed program and accreditation requirements for grain moisture and petroleum quality programs. The directory in Part II also includes information regarding the services available in these areas.

\section{Proposed Grain Moisture Laboratory Program and Accreditation Requirements}

The objectives of developing a Grain Moisture Laboratory Accreditation Program are to promote uniformity in grain moisture testing and provide a accreditation program for State (government) Grain Moisture laboratories.

General requirements for accreditation of Grain Moisture Laboratories will be developed by consensus between participants from State government, the Federal Grain Inspection Service (FGIS) of the U.S. Department of Agriculture, the National Conference on Weights and Measures (NCWM), and the NIST Office of Weights and Measures. The Program will follow the same accreditation/authorization steps as the accreditation of State weights and measures laboratories and the authorization of Participating Laboratories for type evaluation of devices that is described in NBS Handbook 143.

There will be three general requirements for accreditation of State grain moisture laboratories:

(1) The laboratory must be organized and staffed in such a way as to ensure efficient execution of its responsibilities.

The laboratory must have an acceptable facility, appropriate equipment and standards, and trained laboratory staff. The laboratory facilities, equipment and standards, and staff will be described in Handbook 143, Laboratory Program Handbook, and evaluated in a self-appraisal checklist (similar to the 
Appendix $\mathrm{C}$ in that handbook, for weights and measures laboratories). As with the other programs, accreditation is subject to a NIST, FGIS, or other external evaluation.

In addition to the recommended minimum guidelines for facilities, equipment and standards, and staff, the laboratory must have in place, and follow, an appropriate Measurement Control or Quality Control Program.

The laboratory must have a formal, written system description, measurement control, or quality control document that addresses the following accreditation requirements:

Tests must be performed according to nationally recognized (FGIS) specifications.

Documented and recognized test procedures (FGIS), and appropriate test equipment must be used.

Laboratory staff will typically have specialized professional training, and experience in Grain Moisture Testing that is very different from laboratory metrology. No formal training program is in place at NIST at this time and qualifications have not been established. However, staff will be subject to competency testing.

As part of the competency testing, the laboraiory must participate in official round robin measurements (FGIS, NIST) to verify acceptable and consistent test results.

\section{Proposed Petroleum Quality Laboratory Program and Accreditation Requirements}

The objectives of developing a petroleum quality accreditation program are to promote uniformity in petroleum quality testing and provide a accreditation program for State (government) Petroleum Quality laboratories.

General requirements for Petroleum Quality Laboratories will be developed by consensus between participants from State government agencies, the petroleum industry, the National Conference on Weights and Measures (NCWM), the American Society for Testing and Materials (ASTM), and the NIST Office of Weights and Measures recommendations. It will follow the same accreditation/authorization steps as the accreditation of State weights and measures laboratories and the authorization of Participating Laboratories for type evaluation of devices, as those described in NBS Handbook 143.

There will be three general requirements for accreditation of State petroleum quality laboratories:

(1) The laboratory must be organized and staffed in such a way as to ensure efficient execution of its responsibilities.

(2) The laboratory must have an acceptable facility, appropriate equipment and standards and trained laboratory staff. The laboratory facilities, equipment and standards, and staff will be described in Handbook 143, Laboratory Program Handbook, and evaluated in a self-appraisal checklist (similar to that in Appendix $\mathrm{C}$ for weights and measures laboratories). As with the other programs, accreditation is subject to a NIST or other external evaluation.

In addition to the recommended minimum guidelines for facilities, equipment and standards, and staff, the laboratory must have in place, and follow, an appropriate Measurement Control or Quality Control Program.

A formal and written system description, measurement control, or quality control document must be in place that addresses the following accreditation requirements:

(1) Tests must be performed according to nationally recognized (ASTM, API, EPA) specifications.

(2) Documented and recognized test procedures (usually ASTM petroleum test methods), and appropriate test equipment must be used. 
Laboratory staff will typically have professional training, education, and experience in Petroleum Quality Testing, that is very different from laboratory metrology. No formal training programs are in place at NIST at this time and qualifications have not been established. However, under this program, staff will be subject to competency testing.

As part of the competency testing, the laboratory must participate in official round robin measurements (ASTM or regional) to verify acceptable and consistent test results.

\section{Summary}

In order to be accredited in a particular measurement area, a State must have a trained metrologist and an adequate facility, and must demonstrate on a continuing basis that it is capable of providing valid measurements. Accreditation by NIST indicates that the laboratory is capable of providing a measurement service, but each State must verify its own measurement traceability.

The directory of laboratories in Part II summarizes the accredited areas of measurement that each State is qualified to perform and provides detailed information to assist industry and government agencies in locating laboratories that provide needed measurement services. The directory also includes listings for some measurement areas for which accreditation is not currently available. 


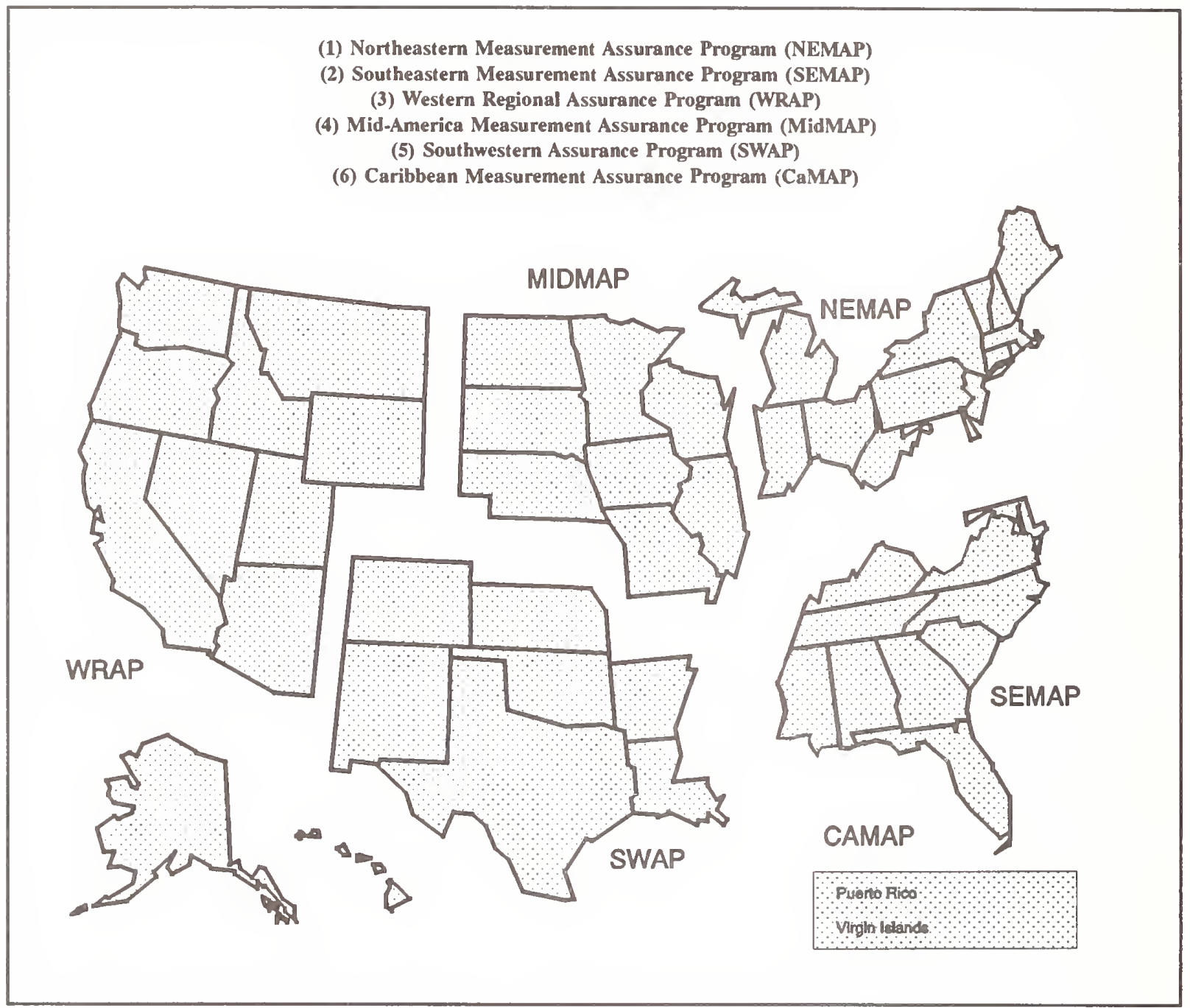

Figure 1. Regional Measurement Management Programs (RMMPs, see p. 5). 


\section{References}

[1] Oppermann, H. V., editor; Specifications, Tolerances, and Other Technical Requirements for Weighing and Measuring Devices; NIST Handbook 44, 1992.

[2] NIST Handbook 105-1 (Revised 1990), Specifications and Tolerances for Reference Standards and Field Standard Weights and Measures. 1. Specifications and Tolerances for Field Standard Weights (NIST Class F).

[3] ASTM (American Society for Testing and Materials) E 617-91, Standard Specification for Laboratory Weights and Precision Mass Standards, ASTM, Philadelphia, PA 19103.

[4] NBS Circular 547, Section 1, Precision Laboratory Standards of Mass and Laboratory Weights, 1954, out of print.

[5] NBS Circular 3, Design and Test of Standards of Mass, out of print.

[6] OIML (International Organization of Legal Metrology), International Recommendation Numbers 1, 2 , $20,25,47,52$.

[7] NBS Handbook 105-2 (Revised 1971), Specifications and Tolerances for Reference Standards and Field Standard Weights and Measures. 2. Specifications and Tolerances for Field Standard Measuring Flask.

[8] NBS Handbook 105-3 (Revised 1979), Specifications and Tolerances for Reference Standards and Field Standard Weights and Measures. 3. Specifications and Tolerances for Graduated Neck Type Volumetric Field Standards.

[9] Oppermann, H. V., Taylor, J. K., State Weights and Measures Laboratories, Program Handbook, NBS/HB 143 (1985).

[10] Taylor, J. K., Oppermann, H. V., Handbook for the Quality Assurance of Metrological Measurements, NBS/HB 145 (1986). 



\section{PART II - Laboratory Directory}

\section{Summary of Measurement Services and Programs}

A table summarizing the available services is provided.

\section{Alphabetical Index}

The following information is given for each State or accredited laboratory (in alphabetical order):

(1) the accreditation period, if accredited by NIST;

(2) the laboratory staff member(s), address, and phone number;

(3) services available; and

(4) fees, if any, for services. 


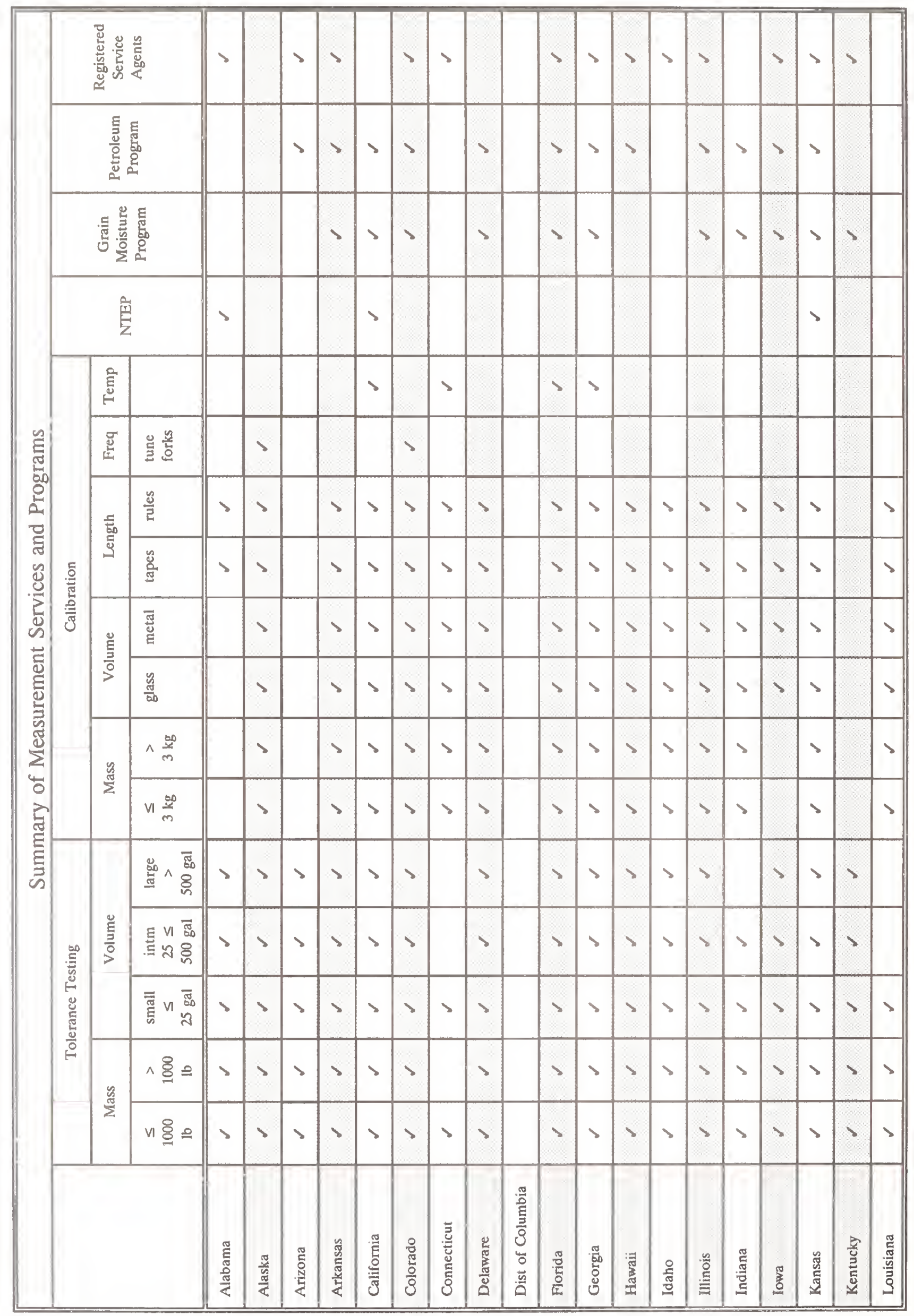




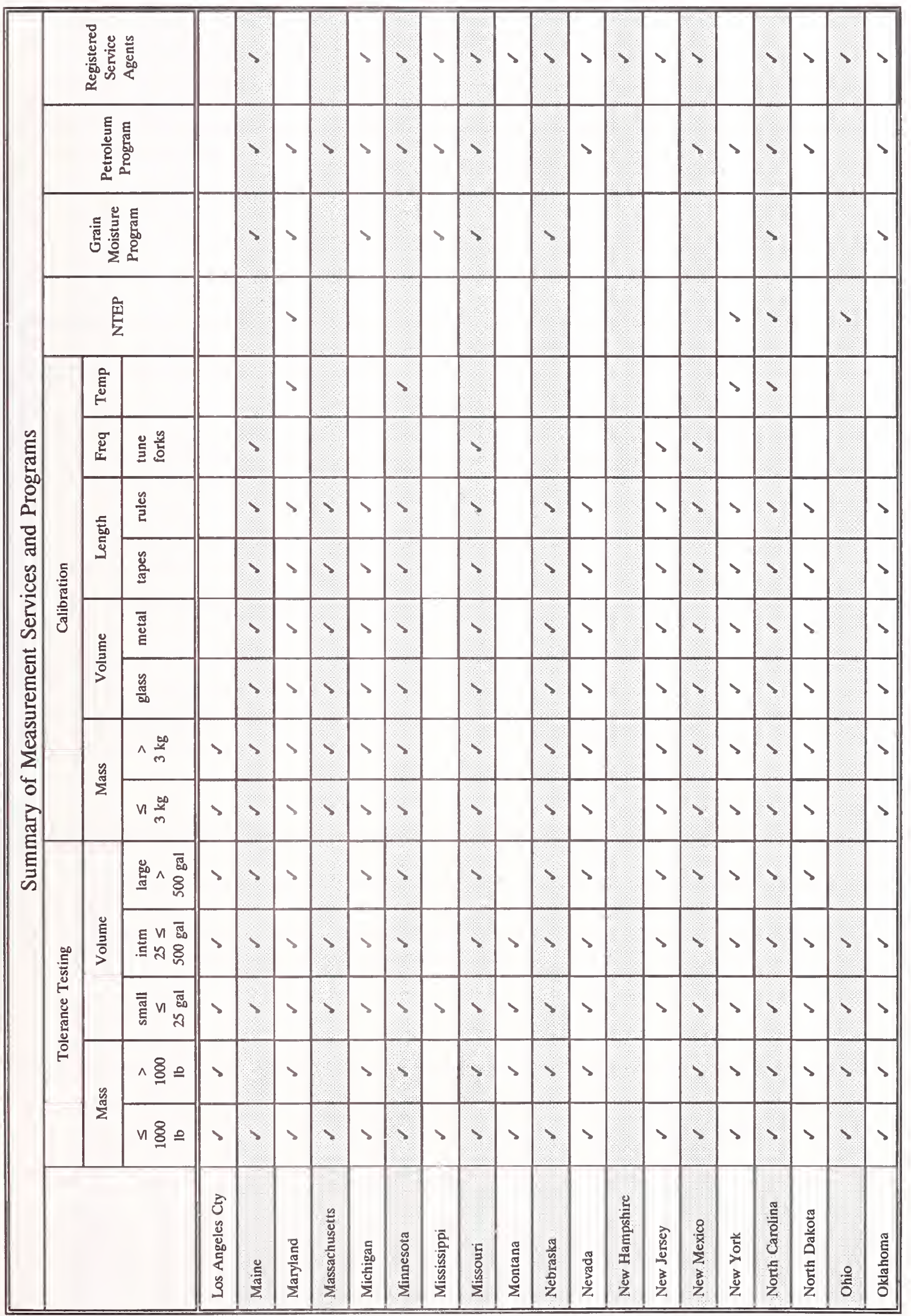




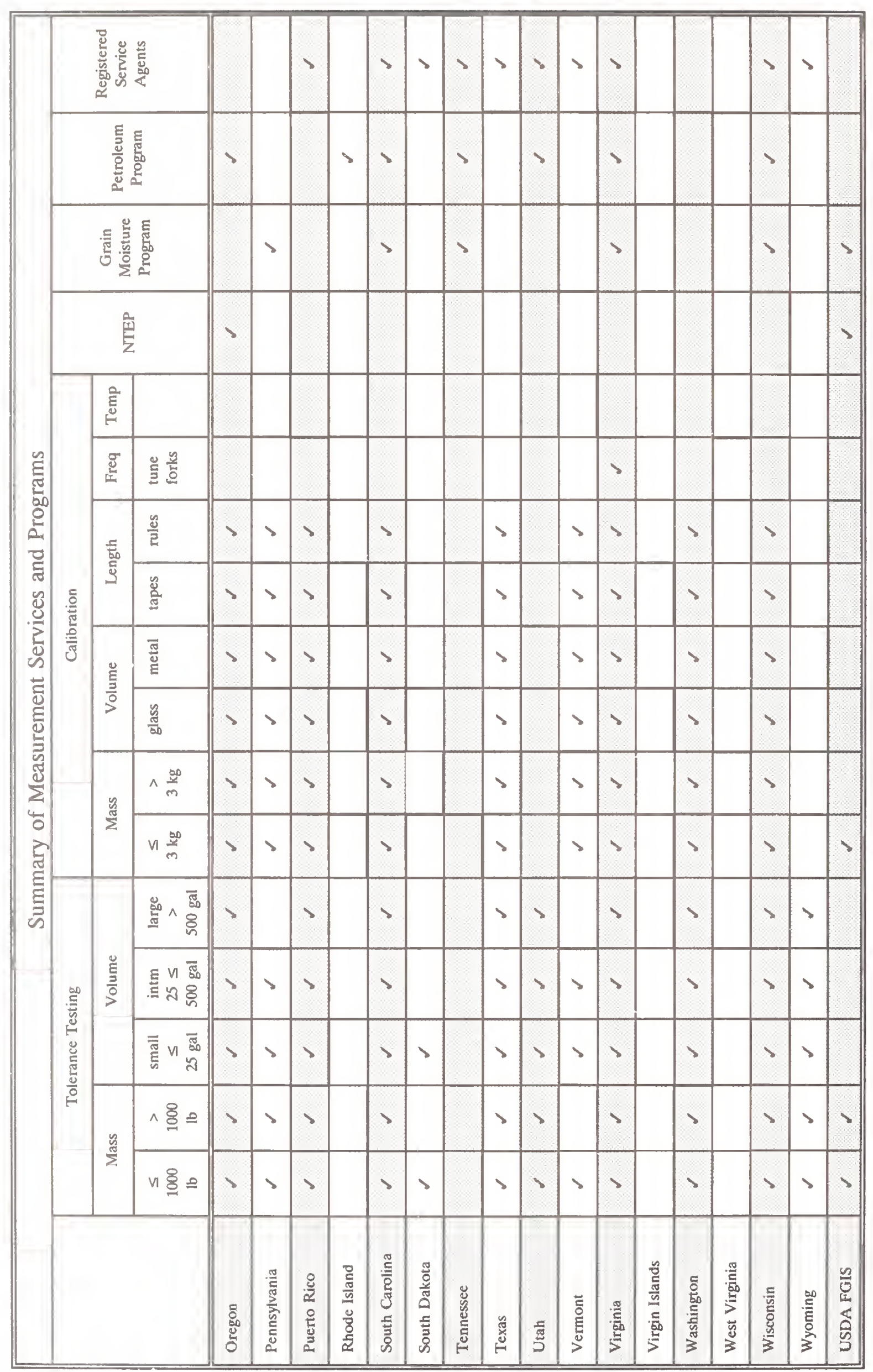




\section{Alabama}

Accreditation Period: 1993

\begin{tabular}{||l||l|l||}
\hline \hline Laboratory Staff & Address & Telephone Number \\
\hline \hline John W. Campbell, Metrologist & $\begin{array}{l}\text { Division of Gins \& Warehouses } \\
\text { PO Box 3336 } \\
\text { Montgomery, AL 36193 Snow, Metrologist }\end{array}$ & FAX: (205) 240-3135 \\
& & \\
\hline
\end{tabular}

\begin{tabular}{|c|c|c|c|c|c|}
\hline \multicolumn{6}{|c|}{ Services Available } \\
\hline \multicolumn{3}{|c|}{ Tolerance Testing } & \multicolumn{3}{|c|}{ Calibration } \\
\hline \multirow[t]{2}{*}{ Mass } & Weight $\leq 1000 \mathrm{lb}$ & $\mathrm{F}$ & \multirow[t]{2}{*}{ Mass } & Weight $\leq 3 \mathrm{~kg}$ & $\mathrm{x}$ \\
\hline & Weight $>1000 \mathrm{lb}$ & F & & Weight $>3 \mathrm{~kg}$ & $\mathrm{x}$ \\
\hline \multirow[t]{3}{*}{ Volume } & Small $\leq 25$ gal & F & \multirow[t]{3}{*}{ Volume } & Metal Standards & $\mathrm{x}$ \\
\hline & Intermediate $25-500$ gal & $\mathbf{F}$ & & \multirow[t]{2}{*}{ Small Glassware } & \multirow[t]{2}{*}{$\mathrm{x}$} \\
\hline & Large $>500$ gal & $F$ & & & \\
\hline \multirow{4}{*}{$\begin{array}{l}\text { O: } \\
\text { F: } \\
\text { N: } \\
\text { X: }\end{array}$} & \multirow{4}{*}{\multicolumn{2}{|c|}{$\begin{array}{l}\text { Service to parent organization only; } \\
\text { Service available on a fee basis; } \\
\text { Service available, no fee; } \\
\text { No accreditation, contact laboratory for availability. }\end{array}$}} & \multirow[t]{2}{*}{ Length } & Steel Tapes & $\mathrm{x}$ \\
\hline & & & & Rigid Rules & $\mathrm{x}$ \\
\hline & & & Frequency & Tuning Forks & $\mathrm{x}$ \\
\hline & & & Temperature & Liquid in Glass Thermometry & $\mathrm{x}$ \\
\hline
\end{tabular}

\section{Fees}

Labor...\$45 per hour. Registered service agents and parent organization are exempt from fees. 


\section{Alabama}

\begin{tabular}{||l|l||}
\hline \multicolumn{2}{|c||}{ Grain Moisture Testing } \\
\hline \hline Address & Telephone Number \\
\hline \hline NOT AVAILABLE & \\
\hline Person responsible for program & \\
\hline Contact for additional information & \\
\hline Additional accreditation/recognition & \\
\hline
\end{tabular}

\begin{tabular}{||l|l||}
\hline \multicolumn{2}{|c|}{ Petroleum Quality Testing } \\
\hline \hline Address & Telephone Number \\
\hline \hline $\begin{array}{l}\text { Division of Agricultural Chemistry } \\
\text { Department of Agriculture \& Industries } \\
\text { PO Box 3336 } \\
\text { Montgomery, AL 36109.0336 }\end{array}$ & (205) 242-2639 \\
\hline Person responsible for program & \\
\hline Contact for additional information & Lance Hester \\
\hline Additional accreditation/recognition & Vaughn Johnson \\
\hline
\end{tabular}

\begin{tabular}{||l|l||}
\hline \multicolumn{2}{|c|}{ National Type Evaluation Program (NTEP) } \\
\hline \hline Address & Telephone Number \\
\hline \hline $\begin{array}{l}\text { Division of Gins \& Warehouses } \\
\text { PO Box 3336 } \\
\text { Montgomery, AL 36109-0336 }\end{array}$ & $(205)$ 242-2639 \\
\hline Person responsible for program & Aubrey C. Dismukes \\
\hline Contact for additional information & Larry Turberville, John Campbell \\
\hline Additional accreditation/recognition & NIST Authorized Laboratory \\
\hline NTEP performance testing of vehicle, axle-load, livestock, and floor scales. \\
\hline
\end{tabular}




\section{Alaska}

Accreditation Period: 1991-1993

\begin{tabular}{|c|c|c|}
\hline Laboratory Staff & Address & Telephone Number \\
\hline Darrel E. Cavender, Metrologist & $\begin{array}{l}\text { Dept of Comm \& Economic Dev } \\
\text { Measurement Standards } \\
12050 \text { Industry Way, Bldg "O" } \\
\text { Anchorage, AK } 99515 \\
\text { PO Box } 111686 \\
\text { Anchorage, AK } 99511\end{array}$ & $\begin{array}{l}(907) 345-7750 \\
\text { FAX: }(907) 345-2641 \\
\text { or }(907) 345-6835\end{array}$ \\
\hline
\end{tabular}

\begin{tabular}{|c|c|c|c|c|c|}
\hline \multicolumn{6}{|c|}{ Services Available } \\
\hline \multicolumn{3}{|c|}{ Tolerance Testing } & \multicolumn{3}{|c|}{ Calibration } \\
\hline \multirow[t]{2}{*}{ Mass } & Weight $\leq 1000 \mathrm{lb}$ & N & \multirow[t]{2}{*}{ Mass } & Weight $\leq 3 \mathrm{~kg}$ & $\mathrm{~N}$ \\
\hline & Weight $>1000 \mathrm{lb}$ & $\mathrm{N}$ & & Weight $>3 \mathrm{~kg}$ & $\mathrm{~N}$ \\
\hline \multirow[t]{3}{*}{ Volume } & Small $\leq 25$ gal & N & \multirow[t]{3}{*}{ Volume } & Metal Standards & $\mathrm{N}$ \\
\hline & Intermediate $25-500 \mathrm{gal}$ & $\mathrm{N}$ & & Small Glassware & $\mathrm{N}$ \\
\hline & Large $>500 \mathrm{gal}$ & $\mathrm{N}$ & & & \\
\hline \multirow{4}{*}{$\begin{array}{l}\text { O: } \\
\text { F: } \\
\text { N: } \\
\text { X: }\end{array}$} & \multirow{4}{*}{\multicolumn{2}{|c|}{$\begin{array}{l}\text { Service to parent organization only; } \\
\text { Service available on a fee basis; } \\
\text { Service available, no fee; } \\
\text { No accreditation, contact laboratory for availability. }\end{array}$}} & \multirow[t]{2}{*}{ Length } & Steel Tapes & $\mathrm{N}$ \\
\hline & & & & Rigid Rules & $\mathrm{N}$ \\
\hline & & & Frequency & Tuning Forks & $\mathrm{N}$ \\
\hline & & & Temperature & Liquid in Glass Thermometry & $\mathrm{x}$ \\
\hline
\end{tabular}

\section{Fees}

No fees are charged. 


\section{Alaska}

\begin{tabular}{|l|l||}
\hline \multicolumn{2}{|c||}{ Grain Moisture Testing } \\
\hline \hline Address & Telephone Number \\
\hline \hline NOT AVAILABLE & \\
\hline Person responsible for program & \\
\hline Contact for additional information & \\
\hline Additional accreditation/recognition & \\
\hline
\end{tabular}

\begin{tabular}{|l|l||}
\hline \multicolumn{2}{|c|}{ Petroleum Quality Testing } \\
\hline \hline Address & Telephone Number \\
\hline \hline NOT AVAILABLE & \\
& \\
\hline Person responsible for program & \\
\hline Contact for additional information & \\
\hline Additional accreditation/recognition & \\
\hline
\end{tabular}

\begin{tabular}{||l|l||}
\hline \multicolumn{2}{|c|}{ Additional Tests or Laboratory Functions } \\
\hline \hline Address & Telephone Number \\
\hline \hline $\begin{array}{l}12050 \text { Industry Way } \\
\text { Anchorage, AK } 99515\end{array}$ & $(907) 345-7750$ \\
\hline Person responsible for program & \\
\hline Contact for additional information & Darrel E. Cavender \\
\hline Additional accreditation/recognition & \\
\hline
\end{tabular}




\section{Arizona}

Accreditation Period: 1993

\begin{tabular}{||l||l|l||}
\hline \hline Laboratory Staff & Address & Telephone Number \\
\hline \hline Kelleen K. Moody, Metrologist & $\begin{array}{l}\text { Dept of Weights and Measures } \\
\text { 9535 E. Doubletree Ranch Rd. } \\
\text { Scottsdale, AZ 85258-5539 }\end{array}$ & FAX: (602) 255-1950 \\
& & \\
\hline
\end{tabular}

\begin{tabular}{|c|c|c|c|c|c|}
\hline \multicolumn{6}{|c|}{ Services Available } \\
\hline \multicolumn{3}{|c|}{ Tolerance Testing } & \multicolumn{3}{|c|}{ Calibration } \\
\hline \multirow[t]{2}{*}{ Mass } & Weight $\leq 1000 \mathrm{lb}$ & F & \multirow[t]{2}{*}{ Mass } & Weight $\leq 3 \mathrm{~kg}$ & $\mathrm{~F}$ \\
\hline & Weight $>1000 \mathrm{lb}$ & F & & Weight $>3 \mathrm{~kg}$ & $\mathrm{~F}$ \\
\hline \multirow[t]{3}{*}{ Volume } & Small $\leq 25$ gal & F & \multirow[t]{3}{*}{ Volume } & Metal Standards & $\mathrm{F}$ \\
\hline & Intermediate $25-500$ gal & F & & Small Glassware & $\mathrm{F}$ \\
\hline & Large $>500 \mathrm{gal}$ & $\mathrm{x}$ & & & \\
\hline \multirow{4}{*}{$\begin{array}{l}\text { O: } \\
\text { F: } \\
\text { N: } \\
\text { X: }\end{array}$} & \multirow{4}{*}{\multicolumn{2}{|c|}{$\begin{array}{l}\text { Service to parent organization only, } \\
\text { Service available on a fee basis; } \\
\text { Service available, no fee; } \\
\text { No accreditation, contact laboratory for availability. }\end{array}$}} & \multirow[t]{2}{*}{ Length } & Steel Tapes & $\mathrm{F}$ \\
\hline & & & & Rigid Rules & $\mathrm{F}$ \\
\hline & & & Frequency & Tuning Forks & $\mathrm{X}$ \\
\hline & & & Temperature & Liquid in Glass Thermometry & $\mathrm{x}$ \\
\hline
\end{tabular}

\section{Fees}

Labor ... $\$ 40$ per hour. Minimum half-hour charge of $\$ 24$.

\section{Remarks:}

The Arizona laboratory moved into a new facility during 1993. 


\section{Arizona}

\begin{tabular}{|l|l||}
\hline \multicolumn{2}{|c|}{ Grain Moisture Testing } \\
\hline \hline Address & Telephone Number \\
\hline NOT AVAILABLE & \\
\hline Person responsible for program & \\
\hline Contact for additional information & \\
\hline Additional accreditation/recognition & \\
\hline
\end{tabular}

\begin{tabular}{||l|l||}
\hline \multicolumn{2}{|c|}{ Petroleum Quality Testing } \\
\hline \hline Address & Telephone Number \\
\hline \hline $\begin{array}{l}\text { 1951 West North Lane } \\
\text { Phoenix, AZ 85021 }\end{array}$ & $(602) 255-5211$ \\
\hline Person responsible for program & \\
\hline Contact for additional information & John Hays \\
\hline Adđitional accreditation/recognition & Jim Webbert \\
\hline
\end{tabular}

\begin{tabular}{||l|l||}
\hline \multicolumn{2}{|c|}{ Additional Tests or Laboratory Functions } \\
\hline \hline Address & Telephone Number \\
\hline \hline $\begin{array}{l}\text { 1951 West North Lane } \\
\text { Phoenix, AX 85021 }\end{array}$ & $(602) 255-5211$ \\
\hline Person responsible for program & John Hays \\
\hline Contact for additional information & Kelleen K. Moody \\
\hline Additional accreditation/recognition & \\
\hline
\end{tabular}




\section{Arkansas}

Accreditation Period: 1993-1995

\begin{tabular}{|l||l|l|}
\hline Laboratory Staff & Address & Telephone Number \\
\hline \hline Billy W. Sullivant, Laboratory Supervisor & & Arkansas Bureau of Standards \\
Charles Kirspel, Jr., Metrologist & Little Rock, AR 72209 & FAX: (501) 562-7605 \\
Randall W. Burns, Metrologist \& Grain & & \\
\hline
\end{tabular}

\begin{tabular}{|c|c|c|c|c|c|}
\hline \multicolumn{6}{|c|}{ Services Available } \\
\hline \multicolumn{3}{|c|}{ Tolerance Testing } & \multicolumn{3}{|c|}{ Calibration } \\
\hline \multirow[t]{2}{*}{ Mass } & Weight $\leq 1000 \mathrm{lb}$ & $\mathrm{N}$ & \multirow[t]{2}{*}{ Mass } & Weight $\leq 3 \mathrm{~kg}$ & $\mathrm{~N}$ \\
\hline & Weight $>1000 \mathrm{lb}$ & $\mathrm{N}$ & & Weight $>3 \mathrm{~kg}$ & $\mathrm{~N}$ \\
\hline \multirow[t]{3}{*}{ Volume } & Small $\leq 25$ gal & $\mathrm{N}$ & \multirow[t]{3}{*}{ Volume } & Metal Standards & $\mathrm{N}$ \\
\hline & Intermediate 25 - $500 \mathrm{gal}$ & $\mathrm{N}$ & & Small Glassware & $\mathrm{N}$ \\
\hline & Large $>500$ gal & $\mathrm{N}$ & & & \\
\hline \multirow{4}{*}{$\begin{array}{l}\text { O: } \\
\text { F: } \\
\text { N: } \\
X:\end{array}$} & \multirow{4}{*}{\multicolumn{2}{|c|}{$\begin{array}{l}\text { Service to parent organization only; } \\
\text { Service available on a fee basis; } \\
\text { Service available, no fee; } \\
\text { No accreditation, contact laboratory for availability. }\end{array}$}} & \multirow[t]{2}{*}{ Length } & Steel Tapes & $\mathrm{N}$ \\
\hline & & & & Rigid Rules & $\mathrm{N}$ \\
\hline & & & Frequency & Tuning Forks & $\mathrm{x}$ \\
\hline & & & Temperature & Liquid in Glass Thermometry & $\mathrm{x}$ \\
\hline
\end{tabular}

\section{Fees}

No fees are charged. 


\section{Arkansas}

\begin{tabular}{|l|l||}
\hline \multicolumn{2}{|c||}{ Grain Moisture Testing } \\
\hline Address & Telephone Number \\
\hline $\begin{array}{l}\text { Arkansas Bureau of Standards } \\
4608 \text { West 61st Street } \\
\text { Little Rock, AR 72209 }\end{array}$ & $\begin{array}{l}\text { (501) 324-9680 } \\
\text { FAX: (501) 562-7605 }\end{array}$ \\
\hline Person responsible for program & Billy W. Sullivant \\
\hline Contact for additional information & \\
\hline Additional accreditation/recognition & \\
\hline
\end{tabular}

\begin{tabular}{|l|l||}
\hline \multicolumn{2}{|c|}{ Petroleum Quality Testing } \\
\hline \hline Address & Telephone Number \\
\hline $\begin{array}{l}\text { Arkansas Bureau of Standards } \\
4608 \text { West } 61 \text { st Street } \\
\text { Little Rock, AR 72209 }\end{array}$ & (501) 324-9680 \\
\hline Ferson responsible for program & David $562-7605$ \\
\hline Contact for additional information & \\
\hline Additional accreditation/recognition & ASTM D-2, Chemist, CDL \\
\hline
\end{tabular}

\begin{tabular}{|l|l||}
\hline \multicolumn{2}{|c||}{ Additional Tests or Laboratory Functions } \\
\hline \hline Address & Telephone Number \\
\hline \hline & \\
\hline Person responsible for program & \\
\hline Contact for additional information & \\
\hline Additional accreditation/recognition & \\
\hline
\end{tabular}




\section{California}

Accreditation Period: 1993-1995

\begin{tabular}{||l||l|l||}
\hline \hline Laboratory Staff & Address & Telephone Number \\
\hline \hline Joseph Rothleder, State Metrologist & Division of Measurement Standards \\
Thomas S. Fletcher, Msmt Stds Specialist III & $\begin{array}{l}\text { Dept of Food and Agriculture } \\
\text { 8500 Fruitridge Rd } \\
\text { Sacramento, CA 95826 }\end{array}$ & FAX: (916) 387-4260 $387-4256$ \\
Walter Camozzi, Msmt Stds Specialist II & & \\
\hline
\end{tabular}

\begin{tabular}{|c|c|c|c|c|c|}
\hline \multicolumn{6}{|c|}{ Services Available } \\
\hline \multicolumn{3}{|c|}{ Tolerance Testing } & \multicolumn{3}{|c|}{ Calibration } \\
\hline \multirow[t]{2}{*}{ Mass } & Weight $\leq 1000 \mathrm{lb}$ & $\mathbf{F}$ & \multirow[t]{2}{*}{ Mass } & Weight $\leq 3 \mathrm{~kg}$ & $\mathbf{F}$ \\
\hline & Weight $>1000 \mathrm{lb}$ & $\mathbf{F}$ & & Weight $>3 \mathrm{~kg}$ & F \\
\hline \multirow[t]{3}{*}{ Volume } & Small $\leq 25$ gal & $\mathrm{F}$ & \multirow[t]{3}{*}{ Volume } & Metal Standards & F \\
\hline & Intermediate 25 - $500 \mathrm{gal}$ & F & & Small Glassware & F \\
\hline & Large $>500 \mathrm{gal}$ & $\mathrm{F}$ & & & \\
\hline \multirow{4}{*}{$\begin{array}{l}\text { O: } \\
\text { F: } \\
\text { N: } \\
X:\end{array}$} & \multirow{4}{*}{\multicolumn{2}{|c|}{$\begin{array}{l}\text { Service to parent organization only; } \\
\text { Service available on a fee basis; } \\
\text { Service available, no fee; } \\
\text { No accreditation, contact laboratory for availability. }\end{array}$}} & \multirow[t]{2}{*}{ Length } & Steel Tapes & F \\
\hline & & & & Rigid Rules & F \\
\hline & & & Frequency & Tuning Forks & $\mathrm{x}$ \\
\hline & & & Temperature & Liquid in Glass Thermometry & $\mathbf{F}$ \\
\hline
\end{tabular}

\section{Fees}

Labor...\$60 per hour, for Metrology Laboratory only. For testing services beyond Metrology Laboratory, contact persons listed 


\section{California}

\begin{tabular}{|c|c|}
\hline \multicolumn{2}{|c|}{ National Type Evaluation Program (NTEP) } \\
\hline Address & Telephone Number \\
\hline $\begin{array}{l}\text { Division of Measurement Standards } \\
8500 \text { Fruitridge Road } \\
\text { Sacramento, CA } 95826\end{array}$ & (916) $387-4241$ \\
\hline Person responsible for program & Steve Cook \\
\hline Contact for additional information & Jim Tollefson \\
\hline Additional accreditation/recognition & NIST Authorized Laboratory \\
\hline \multicolumn{2}{|c|}{$\begin{array}{l}\text { NTEP testing of: } \\
\text { Scales and Weighing Devices } \\
\text { Counter, portable, hanging, floor, crane, hopper, vehicle, axle-load, and livestock scales; automatic bulk weighing systems; } \\
\text { indicating and recording elements, mass flow meters, belt-conveyor scales } \\
\text { Volume Measuring Devices }\end{array}$} \\
\hline \multicolumn{2}{|c|}{$\begin{array}{l}\text { Retail motor-fuel dispensers; indicating and computing elements; vehicle-tank and wholesale meters; service station consoles; LP- } \\
\text { gas liquid meters } \\
\text { Linear Measuring Devices }\end{array}$} \\
\hline
\end{tabular}

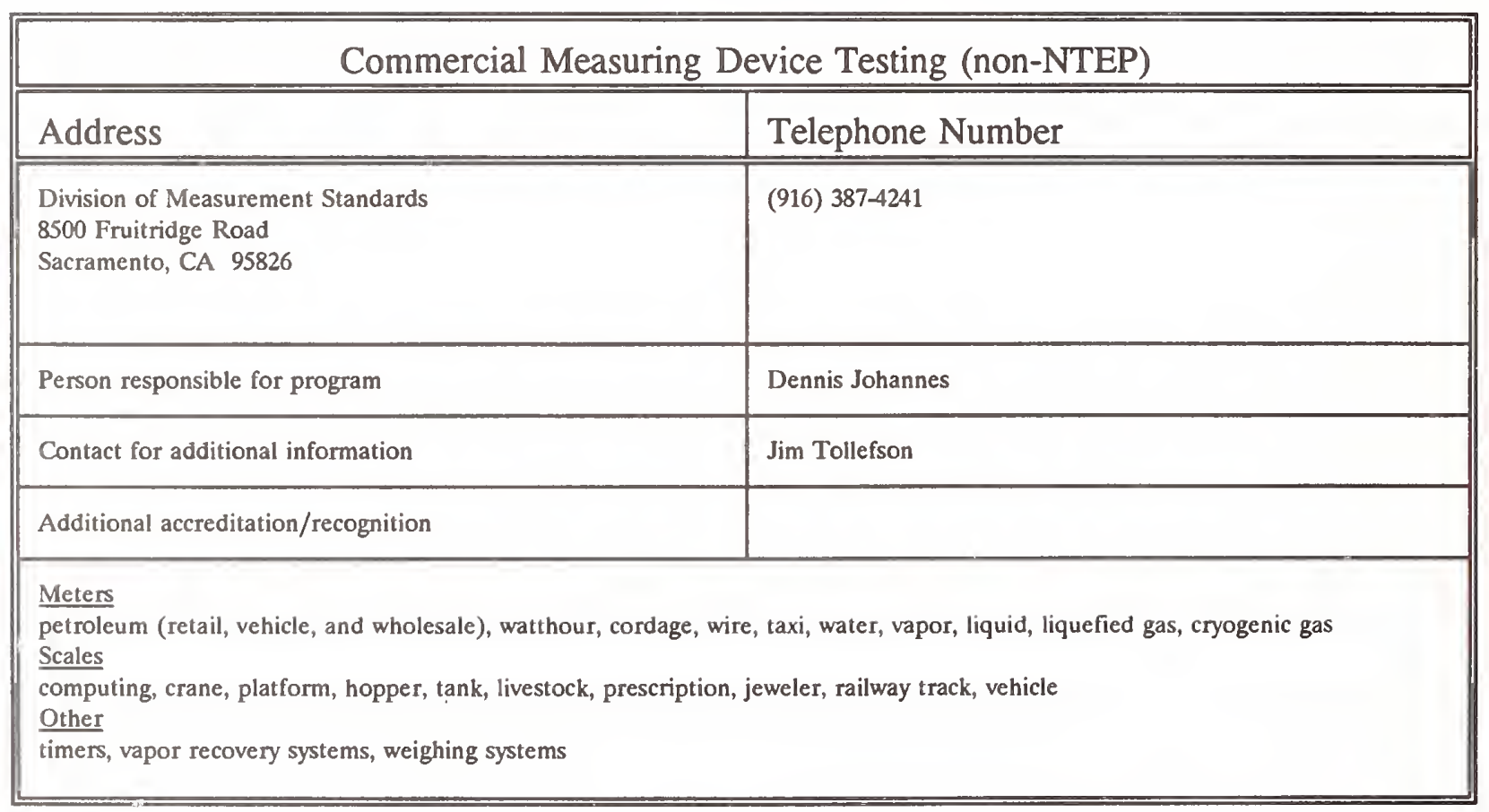




\section{Califormia}

\begin{tabular}{|l|l||}
\hline \multicolumn{2}{|c|}{ Grain Moisture Testing } \\
\hline \hline Address & Telephone Number \\
\hline $\begin{array}{l}\text { Division of Measurement Standards } \\
8500 \text { Fruitridge Road } \\
\text { Sacramento, CA 95826 }\end{array}$ & (916) 387-4241 \\
\hline Person responsible for program & Dennis Johannes \\
\hline Contact for additional information & Walter Camozzi \\
\hline Additional accreditation/recognition & Participation in round robin experiments \\
\hline
\end{tabular}

\begin{tabular}{|l|l||}
\hline \multicolumn{2}{|c||}{ Petroleum Quality Testing } \\
\hline \hline Address & Telephone Number \\
\hline \hline $\begin{array}{l}\text { Division of Measurement Standards } \\
\text { 8500 Fruitridge Road } \\
\text { Sacramento, CA 95826 }\end{array}$ & $(916) 387-4248$ \\
\hline Person responsible for program & Floyd Worcester \\
\hline Contact for additional information & Al Hebert \\
\hline Additional accreditation/recognition & Round robins, Pacific Coast Sample Exchange Program \\
\hline
\end{tabular}

\begin{tabular}{|c|c|}
\hline \multicolumn{2}{|c|}{ Radiation Instrument Calibration } \\
\hline Address & Telephone Number \\
\hline $\begin{array}{l}\text { Office of Emergency Services } \\
2800 \text { Meadowview Road } \\
\text { Sacramento, CA } 95832\end{array}$ & (916) 262-1735 \\
\hline Person responsible for program & John Anderson \\
\hline Contact for additional information & Detmar Schroeder \\
\hline Additional accreditation/recognition & $\begin{array}{l}\text { Accreditation by the Conference of Radiation Control } \\
\text { Program Directors and participates in proficiency tests } \\
\text { conducted by NIST. }\end{array}$ \\
\hline
\end{tabular}





\section{Colorado}

Accreditation Period: 1993-1995

\begin{tabular}{|c|c|c|}
\hline Laboratory Staff & Address & Telephone Number \\
\hline $\begin{array}{l}\text { Jerry S. Wagner, Chief Metrologist } \\
\text { William L. Young, Metrologist }\end{array}$ & $\begin{array}{l}\text { Colorado Dept of Agriculture } \\
\text { Inspection and Consumer Services } \\
\text { Measurement Standards } \\
\text { Metrology Laboratory } \\
3125 \text { Wyandot St } \\
\text { Denver, CO } 80211-3824\end{array}$ & $\begin{array}{l}\text { (303) } 866-2845 \\
\text { FAX: (303) 480-9236 }\end{array}$ \\
\hline
\end{tabular}

\begin{tabular}{|c|c|c|c|c|c|}
\hline \multicolumn{6}{|c|}{ Services Available } \\
\hline \multicolumn{3}{|c|}{ Tolerance Testing } & \multicolumn{3}{|c|}{ Calibration } \\
\hline \multirow[t]{2}{*}{ Mass } & Weight $\leq 1000 \mathrm{lb}$ & F & \multirow[t]{2}{*}{ Mass } & Weight $\leq 3 \mathrm{~kg}$ & $F$ \\
\hline & Weight $>1000 \mathrm{lb}$ & F & & Weight $>3 \mathrm{~kg}$ & F \\
\hline \multirow[t]{3}{*}{ Volume } & Small $\leq 25$ gal & F & \multirow[t]{3}{*}{ Volume } & Metal Standards & F \\
\hline & Intermediate $25-500$ ga & F & & Small Glassware & F \\
\hline & Large > 500 gal & $\mathbf{F}$ & & & \\
\hline \multirow{4}{*}{$\begin{array}{l}\text { O: } \\
\text { F: } \\
\text { N: } \\
X:\end{array}$} & \multirow{4}{*}{\multicolumn{2}{|c|}{$\begin{array}{l}\text { Service to parent organization only; } \\
\text { Service available on a fee basis; } \\
\text { Service available, no fee; } \\
\text { No accreditation, contact laboratory for availability. }\end{array}$}} & \multirow[t]{2}{*}{ Length } & Steel Tapes & F \\
\hline & & & & Rigid Rules & F \\
\hline & & & Frequency & Tuning Forks & $\mathrm{F}$ \\
\hline & & & Temperature & Liquid in Glass Thermometry & $\mathrm{x}$ \\
\hline
\end{tabular}

\section{Fees}

Labor...\$20 per hour.

\section{Remarks:}

Laboratory has been involved with the development of procedures to evaluate laser radar guns and the development of procedures for testing tuning forks. 


\section{Colorado}

\begin{tabular}{||l|l||}
\hline \multicolumn{2}{|c||}{ Grain Moisture Testing } \\
\hline \hline Address & Telephone Number \\
\hline \hline $\begin{array}{l}\text { Colorado Department of Agriculture } \\
\text { Measurement Standards Section } \\
\text { Denver, CO 80211-3824 }\end{array}$ & (303) 866-2845 \\
\hline Person responsible for program & William L. Young \\
\hline Contact for additional information & Richard K. Miller \\
\hline Additional accreditation/recognition & \\
\hline
\end{tabular}

\begin{tabular}{||l|l||}
\hline \multicolumn{2}{|c||}{ Petroleum Quality Testing } \\
\hline \hline Address & Telephone Number \\
\hline $\begin{array}{l}\text { Colorado Department of Labor and Employment } \\
\text { Oil Inspection Section } \\
1001 \text { East 62nd Ave, Bidg } 1 \\
\text { Denver, CO 80216-1140 }\end{array}$ & (303) 289-5643 \\
\hline Person responsible for program & Richard D. Piper \\
\hline Contact for additional information & Helen Lois Struck \\
\hline Additional accreditation/recognition & \\
\hline
\end{tabular}

\begin{tabular}{|l|l|}
\hline \multicolumn{2}{|c|}{ Additional Tests or Laboratory Functions } \\
\hline \hline Address & Telephone Number \\
\hline $\begin{array}{l}\text { Colorado Department of Agriculture } \\
\text { Measurement Standards Section } \\
\text { 3125 Wyandot Street } \\
\text { Denver, CO 80211-3824 }\end{array}$ & $(303)$ 866-2845 \\
\hline Person responsible for program & Jerry S. Wagner \\
\hline Contact for additional information & William L. Young \\
\hline Additional accreditation/recognition & \\
\hline
\end{tabular}




\section{Connecticut}

Accreditation Period: 1991-1993

\begin{tabular}{||l|l|l||}
\hline Laboratory Staff & Address & Telephone Number \\
\hline \hline Michael J. Dynia, Metrologist & Weights and Measures Division \\
& $\begin{array}{l}\text { Dept of Consumer Protection } \\
\text { State Office Bldg Room G15 } \\
\text { Hartford, CT 06106 }\end{array}$ & $\begin{array}{l}\text { (203) 566-5230 Office, Rm G-31A } \\
\text { FAX: (203) 566-7630 Lab, Rm G-15 }\end{array}$ \\
\hline
\end{tabular}

\begin{tabular}{|c|c|c|c|c|c|}
\hline \multicolumn{6}{|c|}{ Services Available } \\
\hline \multicolumn{3}{|c|}{ Tolerance Testing } & \multicolumn{3}{|c|}{ Calibration } \\
\hline \multirow[t]{2}{*}{ Mass } & Weight $\leq 1000 \mathrm{lb}$ & F & \multirow[t]{2}{*}{ Mass } & Weight $\leq 3 \mathrm{~kg}$ & F \\
\hline & Weight $>1000 \mathrm{lb}$ & $\mathrm{x}$ & & Weight $>3 \mathrm{~kg}$ & F \\
\hline \multirow[t]{3}{*}{ Volume } & Small $\leq 25$ gal & $\mathbf{F}$ & \multirow[t]{3}{*}{ Volume } & Metal Standards & F \\
\hline & Intermediate 25 - $500 \mathrm{gal}$ & $\mathrm{x}$ & & Small Glassware & F \\
\hline & Large $>500 \mathrm{gal}$ & $\mathrm{x}$ & & & \\
\hline \multirow{4}{*}{$\begin{array}{l}\text { O: } \\
\text { F: } \\
\text { N: } \\
\text { X: }\end{array}$} & \multirow{4}{*}{\multicolumn{2}{|c|}{$\begin{array}{l}\text { Service to parent organization only; } \\
\text { Service available on a fee basis; } \\
\text { Service available, no fee; } \\
\text { No accreditation, contact laboratory for availability. }\end{array}$}} & \multirow[t]{2}{*}{ Length } & Steel Tapes & $\mathrm{F}$ \\
\hline & & & & Rigid Rules & F \\
\hline & & & Frequency & Tuning Forks & $\mathrm{x}$ \\
\hline & & & Temperature & Liquid in Glass Thermometry & F \\
\hline
\end{tabular}

\section{Fees}

Labor...\$75 per hour. Minimum charge \$75. Certificate of Traceability: per weight kit No fees charged pursuant to CT General Statutes, Section 43-50.

Tolerance testing rates:

$50 \mathrm{lb}(25 \mathrm{~kg})$ to $1000 \mathrm{lb}(500 \mathrm{~kg}) \$ 25$ each

$2 \mathrm{lb}(1 \mathrm{~kg})$ to $50 \mathrm{lb}(20 \mathrm{~kg}) \$ 13$ each

less than $2 \mathrm{lb}(1 \mathrm{~kg}) \quad \$ 7.00$ each

10 to $32 \mathrm{lb}$ weight kits ( $<26$ pieces) $\$ 95$ per kit

Other weight kits ( $<16$ pieces) $\$ 70$ per kit

\section{Remarks:}

Laboratory tests clinical thermometers. 


\section{Connecticut}

\begin{tabular}{||l|l||}
\hline \multicolumn{2}{|c|}{ Grain Moisture Testing } \\
\hline \hline Address & Telephone Number \\
\hline \hline $\begin{array}{l}\text { Department of Agriculture } \\
\text { Rm 287, 165 Capitol Avenue } \\
\text { Hartford, CT } 06106\end{array}$ & (203) 566-5268 \\
\hline Person responsible for program & \\
\hline Contact for additional information & Al VanDyke \\
\hline Additional accreditation/recognition & \\
\hline
\end{tabular}

\begin{tabular}{||l|l||}
\hline \multicolumn{2}{|c|}{ Petroleum Quality Testing } \\
\hline \hline Address & Telephone Number \\
\hline \hline $\begin{array}{l}\text { Department of Consumer Protection } \\
\text { Rm G-17, 65 Capitol Avenue } \\
\text { Hartford, CT 06106 }\end{array}$ & $\begin{array}{l}\text { (203) } 566-5230 \\
\text { (203) } 566-4778\end{array}$ \\
\hline Person responsible for program & Allan M. Nelson \\
\hline Contact for additional information & Raymond Kalentkowski \\
\hline Additional accreditation/recognition & \\
\hline
\end{tabular}

\begin{tabular}{||l|l||}
\hline \multicolumn{2}{|c||}{ Additional Tests or Laboratory Functions } \\
\hline \hline Address & Telephone Number \\
\hline \hline & \\
& \\
\hline Person responsible for program & \\
\hline Contact for additional information & \\
\hline Additional accreditation/recognition & \\
\hline \hline
\end{tabular}




\section{Delaware}

Accreditation Period: 1993-1995

\begin{tabular}{||l|l|l||}
\hline \hline Laboratory Staff & Address & Telephone Number \\
\hline \hline David L. Baird, Metrologist & & (302) 739-4811 X232 \\
& $\begin{array}{l}\text { DE Dept of Agriculture } \\
\text { Weights and Measures Section } \\
\text { 2320 S DuPont Hwy } \\
\text { Dover, DE 19901-5515 }\end{array}$ & FAX: (302) 697-6287 \\
& & \\
\hline
\end{tabular}

\begin{tabular}{|c|c|c|c|c|c|}
\hline \multicolumn{6}{|c|}{ Services Available } \\
\hline \multicolumn{3}{|c|}{ Tolerance Testing } & \multicolumn{3}{|c|}{ Calibration } \\
\hline \multirow[t]{2}{*}{ Mass } & Weight $\leq 1000 \mathrm{lb}$ & $\mathrm{N}$ & \multirow[t]{2}{*}{ Mass } & Weight $\leq 3 \mathrm{~kg}$ & $\mathrm{~N}$ \\
\hline & Weight $>1000 \mathrm{lb}$ & N & & Weight $>3 \mathrm{~kg}$ & $\mathbf{N}$ \\
\hline \multirow[t]{3}{*}{ Volume } & Small $\leq 25$ gal & $\mathrm{N}$ & \multirow[t]{3}{*}{ Volume } & Metal Standards & $\mathrm{N}$ \\
\hline & Intermediate 25 - 500 gal & N & & Small Glassware & $\mathrm{N}$ \\
\hline & Large $>500 \mathrm{gal}$ & $\mathrm{N}$ & & & \\
\hline \multirow{4}{*}{$\begin{array}{l}\text { O: } \\
\text { F: } \\
\text { N: } \\
\text { X: }\end{array}$} & \multirow{4}{*}{\multicolumn{2}{|c|}{$\begin{array}{l}\text { Service to parent organization only, } \\
\text { Service available on a fee basis; } \\
\text { Service available, no fee; } \\
\text { No accreditation, contact laboratory for availability. }\end{array}$}} & \multirow[t]{2}{*}{ Length } & Steel Tapes & $\mathrm{N}$ \\
\hline & & & & Rigid Rules & $\mathrm{N}$ \\
\hline & & & Frequency & Tuning Forks & $\mathrm{x}$ \\
\hline & & & Temperature & Liquid in Glass Thermometry & $\mathrm{x}$ \\
\hline
\end{tabular}

\section{Fees}

No fees are charged

\section{Remarks:}

Delaware moved into a new laboratory January 1990 , and uses an environmental chamber for precision mass measurements. 


\section{Delaware}

\begin{tabular}{|l|l||}
\hline \multicolumn{2}{|c||}{ Grain Moisture Testing } \\
\hline \hline Address & Telephone Number \\
\hline \hline $\begin{array}{l}\text { Delaware Department of Agriculture } \\
\text { 2320 S DuPont Hwy } \\
\text { Dover, DE 19901-5515 }\end{array}$ & $(302) 739-4811 \times 232$ \\
\hline Person responsible for program & \\
\hline Contact for additional information & David L. Baird \\
\hline Additional accreditation/recognition & \\
\hline
\end{tabular}

\begin{tabular}{|l|l||}
\hline \multicolumn{2}{|c|}{ Petroleum Quality Testing } \\
\hline \hline Address & Telephone Number \\
\hline \hline $\begin{array}{l}\text { Motor Fuel Tax Administration } \\
\text { PO Drawer E } \\
\text { Dover, DE 19903-1555 }\end{array}$ & $\begin{array}{l}\text { (302) 739-4538 } \\
\text { FAX: (302) 739-6299 }\end{array}$ \\
\hline Person responsible for program & Dale A. Shuirman \\
\hline Contact for additional information & \\
\hline Additional accreditation/recognition & work being done by the State of Maryland \\
\hline
\end{tabular}

\begin{tabular}{|l|l||}
\hline \multicolumn{2}{|c||}{ Additional Tests or Laboratory Functions } \\
\hline \hline Address & Telephone Number \\
\hline \hline & \\
\hline Person responsible for program & \\
\hline Contact for additional information & \\
\hline Additional accreditation/recognition & \\
\hline \hline
\end{tabular}




\section{District of Columbia}

Not currently Accredited

\begin{tabular}{|c|c|c|}
\hline Laboratory Staff & Address & Telephone Number \\
\hline $\begin{array}{l}\text { vacant, Chief/Metrologist } \\
\text { Jeffrey X. Mason, Inspector } \\
\text { Leon Weston, Inspector }\end{array}$ & $\begin{array}{l}\text { Weights, Measures, \& Markets } \\
\text { Branch } \\
614 \text { "H" St NW } \\
\text { Washington, DC } 20001 \\
1110 \text { "U" St SE } \\
\text { Wash, DC } 20020\end{array}$ & (202) $767-7923$ \\
\hline
\end{tabular}

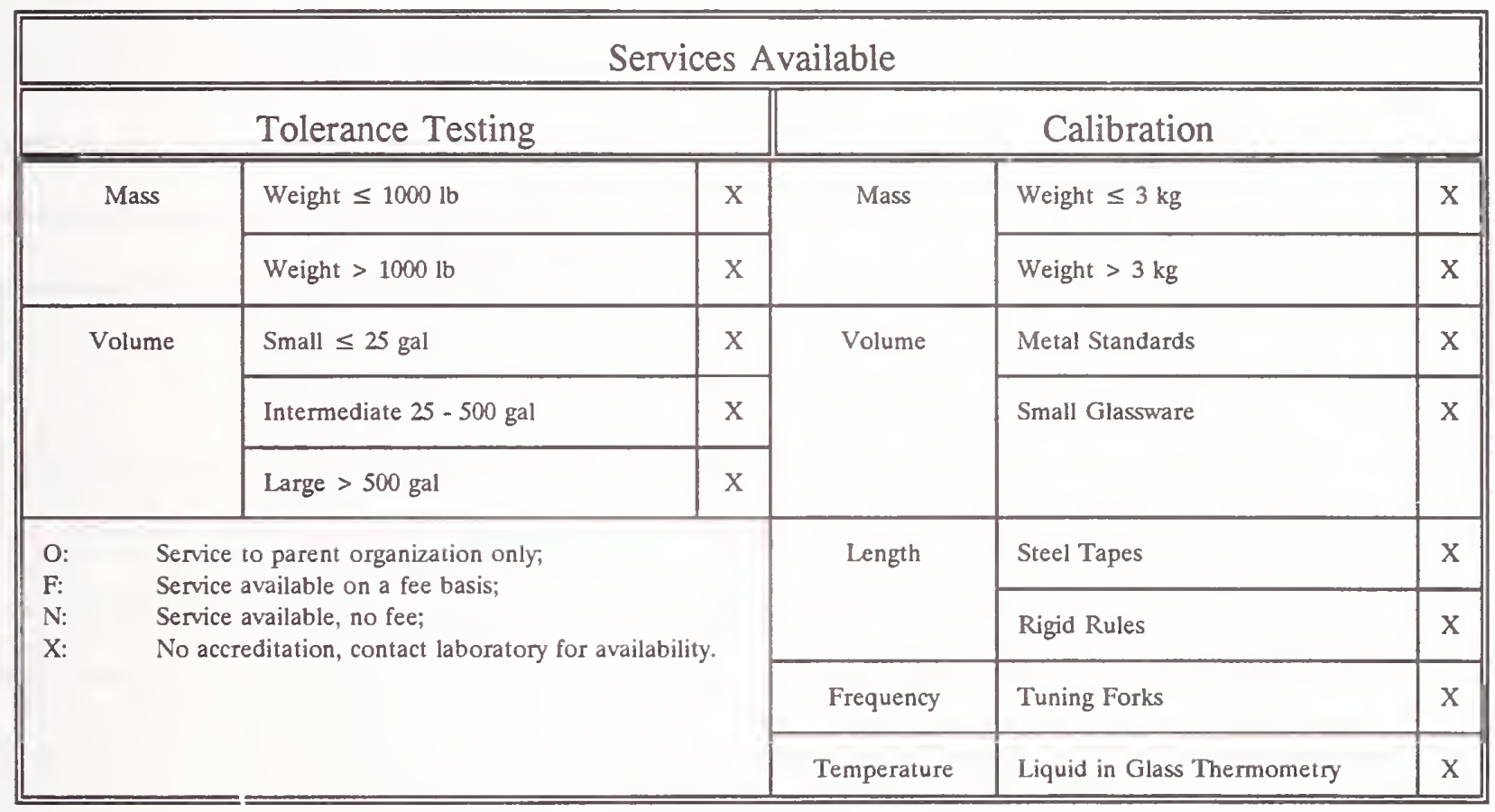

\section{Fees}

Labor...\$15 per hour. 
District of Columbia

\begin{tabular}{||l|l||}
\hline \multicolumn{2}{|c|}{ Grain Moisture Testing } \\
\hline \hline Address & Telephone Number \\
\hline \hline NOT AVAILABLE & \\
& \\
\hline Person responsible for program & \\
\hline Contact for additional information & \\
\hline Additional accreditation/recognition & \\
\hline
\end{tabular}

\begin{tabular}{|l|l||}
\hline \multicolumn{2}{|c|}{ Petroleum Quality Testing } \\
\hline \hline Address & Telephone Number \\
\hline \hline NOT AVAILABLE & \\
& \\
\hline Person responsible for program & \\
\hline Contact for additional information & \\
\hline Additional accreditation/recognition & \\
\hline
\end{tabular}

\begin{tabular}{|l|l||}
\hline \multicolumn{2}{|c|}{ Additional Tests or Laboratory Functions } \\
\hline \hline Address & Telephone Number \\
\hline & \\
& \\
\hline Person responsible for program & \\
\hline Contact for additional information & \\
\hline Additional accreditation/recognition & \\
\hline
\end{tabular}




\section{Florida}

Accreditation Period: 1992-1994

\begin{tabular}{||l|l|l||}
\hline Laboratory Staff & Address & Telephone Number \\
\hline \hline Michael D. Cook, Senior Metrologist & & Department of Agriculture \\
Barry E. Smith, Senior Metrologist & 3125 Conner Blvd, Bldg 2 \\
Tallahassee, FL 32399-1650 & (904) 488-9295 \\
Paul Gentry, Metrologist & & \\
Charles E. Barnes, Lab Technician II & & \\
Carlos D'Arcy, Metrologist, Fort Laud. & & \\
\hline
\end{tabular}

\begin{tabular}{|c|c|c|c|c|c|}
\hline \multicolumn{6}{|c|}{ Services Available } \\
\hline \multicolumn{3}{|c|}{ Tolerance Testing } & \multicolumn{3}{|c|}{ Calibration } \\
\hline \multirow[t]{2}{*}{ Mass } & Weight $\leq 1000 \mathrm{lb}$ & $\mathrm{N}$ & \multirow[t]{2}{*}{ Mass } & Weight $\leq 3 \mathrm{~kg}$ & N \\
\hline & Weight > $1000 \mathrm{lb}$ & $\mathrm{N}$ & & Weight $>3 \mathrm{~kg}$ & $\mathrm{~N}$ \\
\hline \multirow[t]{3}{*}{ Volume } & Small $\leq 25$ gal & $\mathbf{N}$ & \multirow[t]{3}{*}{ Volume } & Metal Standards & $\mathrm{N}$ \\
\hline & Intermediate $25-500 \mathrm{gal}$ & $\mathbf{N}$ & & Small Glassware & N \\
\hline & Large $>500 \mathrm{gal}$ & $\mathbf{N}$ & & & \\
\hline \multirow{4}{*}{$\begin{array}{l}\text { O: } \\
\text { F: } \\
\text { N: } \\
X:\end{array}$} & \multirow{4}{*}{\multicolumn{2}{|c|}{$\begin{array}{l}\text { Service to parent organization only; } \\
\text { Service available on a fee basis; } \\
\text { Service available, no fee"; } \\
\text { No accreditation, contact laboratory for availability. }\end{array}$}} & \multirow[t]{2}{*}{ Length } & Steel Tapes & N \\
\hline & & & & Rigid Rules & $\mathrm{N}$ \\
\hline & & & Frequency & Tuning Forks & $\mathrm{x}$ \\
\hline & & & Temperature & Liquid in Glass Thermometry & $\mathrm{N}$ \\
\hline
\end{tabular}

\begin{tabular}{|l||}
\hline \\
\hline No fees are charged. \\
* Fees have been proposed for late 1993 or early 1994. This will depend on legislative approval. \\
\hline
\end{tabular}

\section{Remarks:}

Laboratory also tests stopwatches. 


\section{Florida}

\begin{tabular}{||l|l||}
\hline \multicolumn{2}{|c||}{ Grain Moisture Testing } \\
\hline \hline Address & Telephone Number \\
\hline \hline $\begin{array}{l}\text { Weights and Measures Laboratory } \\
\text { Bldg \#2 3125 Conner Blvd } \\
\text { Tallahassee, FL 32399-1650 }\end{array}$ & $(904)$ 488-9295 \\
\hline Person responsible for program & Barry E. Smith \\
\hline Contact for additional information & \\
\hline Additional accreditation/recognition & \\
\hline
\end{tabular}

\begin{tabular}{|l|l|}
\hline \multicolumn{2}{|c|}{ Petroleum Quality Testing } \\
\hline \hline Address & Telephone Number \\
\hline \hline $\begin{array}{l}\text { Petroleum Inspection } \\
\text { Bldg \#1 3125 Conner Blvd } \\
\text { Tallahassee, FL 32399-1650 }\end{array}$ & $(904) 488-9740$ \\
\hline $\begin{array}{l}\text { Person responsible for program } \\
\text { Contact for additional information }\end{array}$ & Eric Hamilton \\
\hline Additional accreditation/recognition & Eric Hamilton \\
\hline
\end{tabular}

\begin{tabular}{|l|l||}
\hline \multicolumn{2}{|c|}{ Additional Tests or Laboratory Functions } \\
\hline \hline Address & Telephone Number \\
\hline \hline & \\
\hline Person responsible for program & \\
\hline Contact for additional information & \\
\hline Additional accreditation/recognition & \\
\hline
\end{tabular}




\section{Georgia}

Accreditation Period: 1993-1995

\begin{tabular}{|c|c|c|}
\hline Laboratory Staff & Address & Telephone Number \\
\hline $\begin{array}{l}\text { Martin T. Coile, State Metrologist } \\
\text { Brenda J. Whitener, Asst State Metrologist } \\
\text { Festus Edoimioya, Metrologist II } \\
\text { Valentino Bradford, Metrologist I } \\
\text { Carla Pesce, Metrologist I }\end{array}$ & $\begin{array}{l}\text { Weights and Measures Lab } \\
16 \text { Forest Pkwy } \\
\text { Forest Park, GA } 30050\end{array}$ & $\begin{array}{l}\text { (404) 363-7685 X88 } \\
\text { FAX: (404) 363-7694 }\end{array}$ \\
\hline
\end{tabular}

\begin{tabular}{|c|c|c|c|c|c|}
\hline \multicolumn{6}{|c|}{ Services Available } \\
\hline \multicolumn{3}{|c|}{ Tolerance Testing } & \multicolumn{3}{|c|}{ Calibration } \\
\hline \multirow[t]{2}{*}{ Mass } & Weight $\leq 1000 \mathrm{lb}$ & $\mathbf{F}$ & \multirow[t]{2}{*}{ Mass } & Weight $\leq 3 \mathrm{~kg}$ & $\mathbf{F}$ \\
\hline & Weight > $1000 \mathrm{lb}$ & $\mathbf{F}$ & & Weight $>3 \mathrm{~kg}$ & $\mathbf{F}$ \\
\hline \multirow[t]{3}{*}{ Volume } & Small $\leq 25$ gal & F & \multirow[t]{3}{*}{ Volume } & Metal Standards & $\mathbf{F}$ \\
\hline & Intermediate $25-500 \mathrm{gal}$ & F & & \multirow[t]{2}{*}{ Small Glassware } & \multirow[t]{2}{*}{$\mathbf{F}$} \\
\hline & Large $>500$ gal & $\mathrm{x}$ & & & \\
\hline \multirow{4}{*}{$\begin{array}{l}\text { O: } \\
\text { F: } \\
\text { N: } \\
\text { X: }\end{array}$} & \multirow{4}{*}{\multicolumn{2}{|c|}{$\begin{array}{l}\text { Service to parent organization only; } \\
\text { Service available on a fee basis; } \\
\text { Service available, no fee; } \\
\text { No accreditation, contact laboratory for availability. }\end{array}$}} & \multirow[t]{2}{*}{ Length } & Steel Tapes & $\mathbf{F}$ \\
\hline & & & & Rigid Rules & $\mathbf{F}$ \\
\hline & & & Frequency & Tuning Forks & $\mathrm{x}$ \\
\hline & & & Temperature & Liquid in Glass Thermometry & 0 \\
\hline
\end{tabular}

\section{Fees}

Calibration and tests are prorated at $\$ 25.00$ per hour. Price quotes on request.

\section{Remarks:}

The Georgia laboratory maintains computerized control charts for all weights used in mass calibrations as part of the measurement control program, and has independent laboratory rooms, balances, and standards for calibration and tolerance testing of mass. 


\section{Georgia}

\begin{tabular}{|l|l||}
\hline \multicolumn{2}{|c|}{ Grain Moisture Testing } \\
\hline \hline Address & Telephone Number \\
\hline \hline $\begin{array}{l}\text { Weights and Measures Laboratory } \\
\text { 16 Forest Pkwy } \\
\text { Forest Park, GA 30050 }\end{array}$ & (404) 363-7685 \\
\hline Person responsible for program & Martin T. Coile \\
\hline Contact for additional information & Brenda J. Whitener \\
\hline Additional accreditation/recognition & \\
\hline
\end{tabular}

\begin{tabular}{||l|l||}
\hline \multicolumn{2}{|c|}{ Petroleum Quality Testing } \\
\hline \hline Address & Telephone Number \\
\hline \hline $\begin{array}{l}\text { State Fuel Oil Laboratory } \\
5235 \text { Kennedy Rd } \\
\text { Forest Park, GA 30050 }\end{array}$ & (404) 363-7597 \\
\hline Person responsible for program & Curtis Williams \\
\hline Contact for additional information & \\
\hline Additional accreditation/recognition & \\
\hline
\end{tabular}

\begin{tabular}{|l|l|}
\hline \multicolumn{2}{|c|}{ Additional Tests or Laboratory Functions } \\
\hline \hline Address & Telephone Number \\
\hline \hline & \\
\hline Person responsible for program & \\
\hline Contact for additional information & \\
\hline Additional accreditation/recognition & \\
\hline
\end{tabular}




\section{Hawaii}

Accreditation Period: 1993-1995

\begin{tabular}{|l|l|l||}
\hline \hline Laboratory Staff & Address & Telephone Number \\
\hline Lester Yazawa, Chief Metrologist & $\begin{array}{l}\text { HI Dept of Agriculture } \\
\text { Msmt Standards Division } \\
\text { Donna Kadooka, Metrologist } \\
\text { Honolulu, HI 96813-5524 }\end{array}$ & FAX: (808) 586-0889 \\
& & \\
& & \\
\hline
\end{tabular}

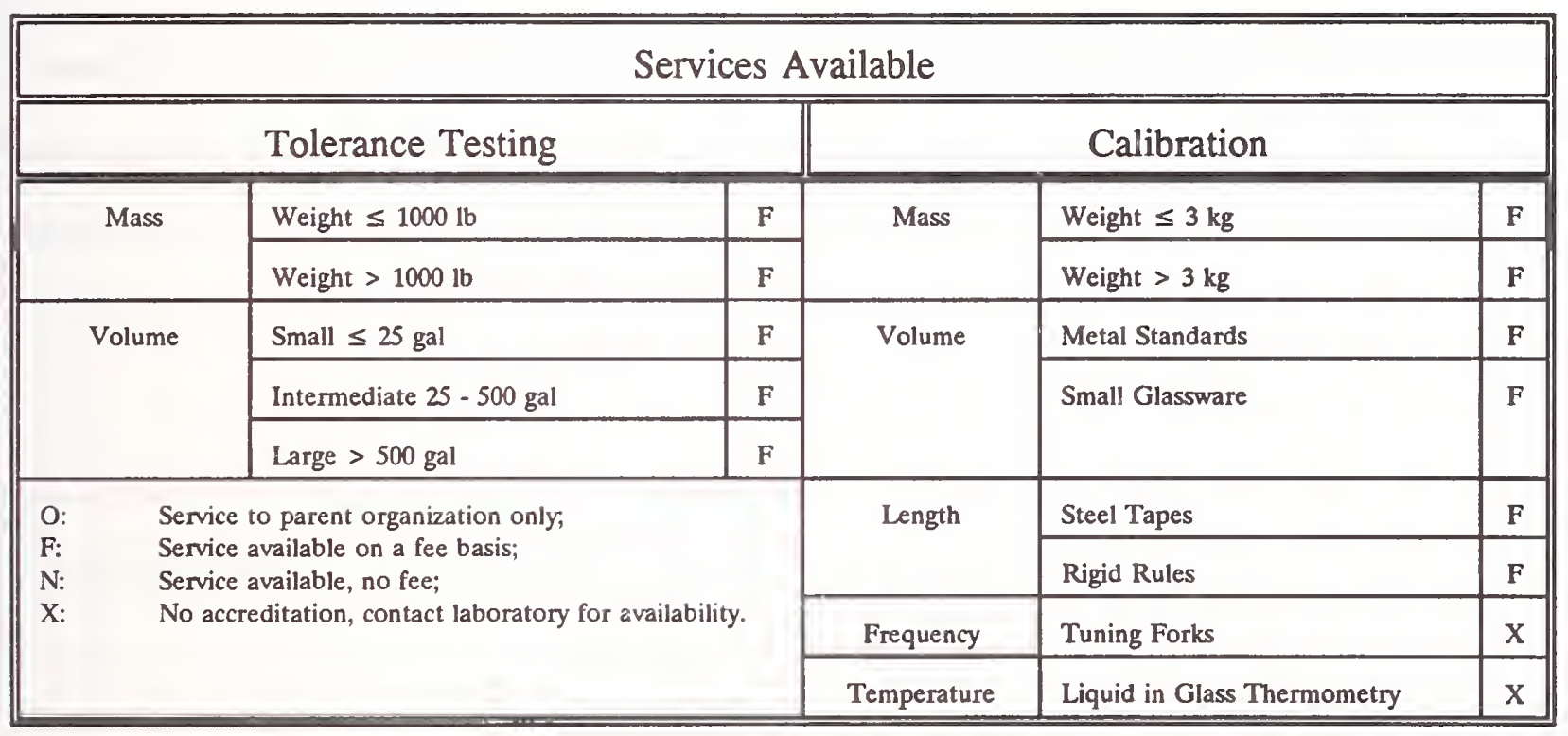

\section{Fees}

Fees are established by rule and vary according to type of artifact and type of service requested. Service repair agencies registered with the state are exempt from fees. 


\section{Hawaii}

\begin{tabular}{||l|l||}
\hline \multicolumn{2}{|c|}{ Grain Moisture Testing } \\
\hline \hline Address & Telephone Number \\
\hline \hline NOT AVAILABLE & \\
\hline Person responsible for program & \\
\hline Contact for additional information & \\
\hline Additional accreditation/recognition & \\
\hline
\end{tabular}

\begin{tabular}{|l|l||}
\hline \multicolumn{2}{|c|}{ Petroleum Quality Testing } \\
\hline \hline Address & Telephone Number \\
\hline \hline $\begin{array}{l}\text { Measurement Standards Division } \\
725 \text { Ilalo St } \\
\text { Honolulu, HI } 96813\end{array}$ & $(808) 586-0882$ \\
\hline Person responsible for program & \\
\hline Contact for additional information & Lester Yazawa \\
\hline Additional accreditation/recognition & \\
\hline
\end{tabular}

\begin{tabular}{|l|l||}
\hline \multicolumn{2}{|c||}{ Additional Tests or Laboratory Functions } \\
\hline \hline Address & Telephone Number \\
\hline & \\
& \\
\hline Person responsible for program & \\
\hline Contact for additional information & \\
\hline Additional accreditation/recognition & \\
\hline
\end{tabular}




\section{Idaho}

Accreditation Period: 1993-1995

\begin{tabular}{||l|l|l||}
\hline Laboratory Staff & Address & Telephone Number \\
\hline \hline Tom W. Schafer, Metrologist & $\begin{array}{l}\text { Dept of Agriculture } \\
\text { Bureau of Weights and Measures } \\
\text { 2216 Kellogg Lane } \\
\text { Boise, ID 83712 }\end{array}$ & FAX: (208) 334-2170 \\
\hline
\end{tabular}

\begin{tabular}{|c|c|c|c|c|c|}
\hline \multicolumn{6}{|c|}{ Services Available } \\
\hline \multicolumn{3}{|c|}{ Tolerance Testing } & \multicolumn{3}{|c|}{ Calibration } \\
\hline \multirow[t]{2}{*}{ Mass } & Weight $\leq 1000 \mathrm{lb}$ & $\mathrm{N}$ & \multirow[t]{2}{*}{ Mass } & Weight $\leq 3 \mathrm{~kg}$ & $\mathrm{~N}$ \\
\hline & Weight $>1000 \mathrm{lb}$ up to $2500 \mathrm{lb}$ & $\mathrm{N}$ & & Weight $>3 \mathrm{~kg}$ & $\mathrm{~N}$ \\
\hline \multirow[t]{3}{*}{ Volume } & Small $\leq 25$ gal & $\mathrm{N}$ & \multirow[t]{3}{*}{ Volume } & Metal Standards & $\mathrm{N}$ \\
\hline & Intermediate 25 - 500 gal & $\mathrm{N}$ & & \multirow[t]{2}{*}{ Small Glassware } & \multirow[t]{2}{*}{$\mathrm{x}$} \\
\hline & Large $>500$ gal & $\mathrm{N}$ & & & \\
\hline \multirow{4}{*}{$\begin{array}{l}\text { O: } \\
\mathrm{F}: \\
\mathrm{N}: \\
\mathrm{X}:\end{array}$} & \multirow{4}{*}{\multicolumn{2}{|c|}{$\begin{array}{l}\text { Service to parent organization only, } \\
\text { Service available on a fee basis; } \\
\text { Service available, no fee; } \\
\text { No accreditation, contact laboratory for availability. }\end{array}$}} & \multirow[t]{2}{*}{ Length } & Steel Tapes & $\mathrm{N}$ \\
\hline & & & & Rigid Rules & $\mathrm{N}$ \\
\hline & & & Frequency & Tuning Forks & $\mathrm{x}$ \\
\hline & & & Temperature & Liquid in Glass Thermometry & $\mathrm{x}$ \\
\hline
\end{tabular}

\section{Fees}

No fees are charged for customers within the state of Idaho. 


\section{Idaho}

\begin{tabular}{|l|l||}
\hline \multicolumn{2}{|c||}{ Grain Moisture Testing } \\
\hline \hline Address & Telephone Number \\
\hline \hline NOT AVAILABLE & \\
\hline Person responsible for program & \\
\hline Contact for additional information & \\
\hline Additional accreditation/recognition & \\
\hline
\end{tabular}

\begin{tabular}{|l|l||}
\hline \multicolumn{2}{|c||}{ Petroleum Quality Testing } \\
\hline \hline Address & Telephone Number \\
\hline \hline NOT AVAILABLE & \\
& \\
\hline Person responsible for program & \\
\hline Contact for additional information & \\
\hline Additional accreditation/recognition & \\
\hline
\end{tabular}

\begin{tabular}{|l|l||}
\hline \multicolumn{2}{|c||}{ Additional Tests or Laboratory Functions } \\
\hline \hline Address & Telephone Number \\
\hline \hline NOT AVAILABLE & \\
\hline Person responsible for program & \\
\hline Contact for additional information & \\
\hline Additional accreditation/recognition & \\
\hline
\end{tabular}




\section{Illinois}

Accreditation Period: 1993-1995

\begin{tabular}{||l|l|l||}
\hline \hline Laboratory Staff & Address & Telephone Number \\
\hline \hline Stephen E. McGuire, Metrologist & & (217) 785-8480 or 8530 \\
Mike Rockford, Metrologist Associate & $\begin{array}{l}\text { IL Dept of Agriculture } \\
\text { Division of Consumer Services } \\
\text { Bureau of Weights and Measures } \\
\text { Standards Laboratories } \\
\text { State Fairgrounds, Box 19281 } \\
\text { Springfield, IL 62794-9281 }\end{array}$ & FAX: (217) 524-6194 \\
& & Toll-Free (Illinois) 1-800-582-0468 \\
\hline
\end{tabular}

\begin{tabular}{|c|c|c|c|c|c|}
\hline \multicolumn{6}{|c|}{ Services Available } \\
\hline \multicolumn{3}{|c|}{ Tolerance Testing } & \multicolumn{3}{|c|}{ Calibration } \\
\hline \multirow[t]{2}{*}{ Mass } & Weight $\leq 1000 \mathrm{lb}$ & F & \multirow[t]{2}{*}{ Mass } & Weight $\leq 3 \mathrm{~kg}$ & F \\
\hline & Weight $>1000 \mathrm{lb}$ & $\mathrm{F}$ & & Weight $>3 \mathrm{~kg}$ & $\mathbf{F}$ \\
\hline \multirow[t]{3}{*}{ Volume } & Small $\leq 25$ gal & $\mathrm{F}$ & \multirow[t]{3}{*}{ Volume } & Metal Standards & F \\
\hline & Intermediate 25 - $500 \mathrm{gal}$ & F & & \multirow[t]{2}{*}{ Small Glassware } & \multirow[t]{2}{*}{$\mathrm{F}$} \\
\hline & Large > 500 gal & $\mathbf{F}$ & & & \\
\hline \multirow{4}{*}{$\begin{array}{l}\text { O: } \\
\text { F: } \\
\text { N: } \\
\text { X: }\end{array}$} & \multirow{4}{*}{\multicolumn{2}{|c|}{$\begin{array}{l}\text { Service to parent organization only; } \\
\text { Service available on a fee basis; } \\
\text { Service available, no fee; } \\
\text { No accreditation, contact laboratory for availability. }\end{array}$}} & \multirow[t]{2}{*}{ Length } & Steel Tapes & $\mathrm{F}$ \\
\hline & & & & Rigid Rules & $\mathrm{F}$ \\
\hline & & & Frequency & Tuning Forks & $\mathrm{X}$ \\
\hline & & & Temperature & Liquid in Glass Thermometry & $\mathrm{X}$ \\
\hline
\end{tabular}

\section{Fees}

Labor...\$25 per hour, tolerance testing.

$\$ 40$ per hour for calibrations in mass, length, and volume.

\section{Remarks:}

The Illinois laboratory has a documented Quality Assurance Program to assure compliance with Mil-Std-45662A audits, and maintains ongoing control charts for all calibrations. 


\section{Illinois}

\begin{tabular}{||l|l||}
\hline \multicolumn{2}{|c|}{ Grain Moisture Testing } \\
\hline \hline Address & Telephone Number \\
\hline $\begin{array}{l}\text { IL Dept of Agriculture } \\
\text { Division of Consumer Services } \\
\text { Bureau of Weights and Measures } \\
\text { State Fairgrounds, Box 19281 } \\
\text { Springfield, IL 62794-9281 }\end{array}$ & (217) 785-8482 \\
\hline Person responsible for program & \\
\hline Contact for additional information & Ron Berg \\
\hline Additional accreditation/recognition & \\
\hline
\end{tabular}

\begin{tabular}{||l|l|}
\hline \multicolumn{2}{|c|}{ Petroleum Quality Testing } \\
\hline \hline Address & Telephone Number \\
\hline $\begin{array}{l}\text { IL Dept of Agriculture } \\
\text { Division of Consumer Services } \\
\text { Bureau of Weights and Measures } \\
\text { State Fairgrounds, Box 19281 } \\
\text { Springfield, IL 62794-9281 }\end{array}$ & (217) 785-8213 \\
\hline Person responsible for program & \\
\hline Contact for additional information & Rich Philmon \\
\hline Additional accreditation/recognition & \\
\hline
\end{tabular}

\begin{tabular}{|l|l|}
\hline \multicolumn{2}{|c|}{ Additional Tests or Laboratory Functions } \\
\hline \hline Address & Telephone Number \\
\hline \hline $\begin{array}{l}\text { IL Dept of Agriculture } \\
\text { Division of Consumer Services } \\
\text { Bureau of Weights and Measures } \\
\text { State Fairgrounds, Box 19281 } \\
\text { Springfield, IL 62794-9281 }\end{array}$ & (217) 782-3817 \\
\hline Person responsible for program & \\
\hline Contact for additional information & Jerry Robertson \\
\hline Additional accreditation/recognition & \\
\hline
\end{tabular}




\section{Indiana}

Accreditation Period: 1993-1994

\begin{tabular}{|c|c|c|}
\hline Laboratory Staff & Address & Telephone Number \\
\hline $\begin{array}{l}\text { Peter W. Boykin, Metrologist } \\
\text { Jerry Clingaman, Asst. Metrologist }\end{array}$ & $\begin{array}{l}\text { Indiana State Board of Health } \\
\text { Division of Weights and Measures } \\
1330 \text { W Michigan St } \\
\text { Indianapolis, IN } 46206\end{array}$ & $\begin{array}{l}\text { (317) 633-0350 (Office) } \\
\text { (317) 633-0789 (Lab) } \\
\text { FAX: (317) 633-0776 }\end{array}$ \\
\hline
\end{tabular}

\begin{tabular}{|c|c|c|c|c|c|}
\hline \multicolumn{6}{|c|}{ Services Available } \\
\hline \multicolumn{3}{|c|}{ Tolerance Testing } & \multicolumn{3}{|c|}{ Calibration } \\
\hline \multirow[t]{2}{*}{ Mass } & Weight $\leq 1000 \mathrm{lb}$ & $\mathrm{N}$ & \multirow[t]{2}{*}{ Mass } & Weight $\leq 3 \mathrm{~kg}$ & $\mathrm{~N}$ \\
\hline & Weight $>1000 \mathrm{lb}$ & $\mathrm{N}$ & & Weight $>3 \mathrm{~kg}$ & $\mathrm{~N}$ \\
\hline \multirow[t]{3}{*}{ Volume } & Small $\leq 25$ gal & $\mathbf{N}$ & \multirow[t]{3}{*}{ Volume } & Metal Standards & $\mathrm{N}$ \\
\hline & Intermediate $25-500 \mathrm{gal}$ & $\mathrm{N}$ & & \multirow[t]{2}{*}{ Small Glassware } & \multirow[t]{2}{*}{$\mathrm{N}$} \\
\hline & Large > $500 \mathrm{gal}$ & $\mathrm{x}$ & & & \\
\hline \multirow{4}{*}{$\begin{array}{l}\text { O: } \\
\text { F: } \\
\text { N: } \\
\text { X: }\end{array}$} & \multirow{4}{*}{\multicolumn{2}{|c|}{$\begin{array}{l}\text { Service to parent organization only, } \\
\text { Service available on a fee basis; } \\
\text { Service available, no fee; } \\
\text { No accreditation, contact laboratory for availability. }\end{array}$}} & \multirow[t]{2}{*}{ Length } & Steel Tapes & $\mathrm{N}$ \\
\hline & & & & Rigid Rules & $\mathrm{N}$ \\
\hline & & & Frequency & Tuning Forks & $\mathrm{x}$ \\
\hline & & & Temperature & Liquid in Glass Thermometry & $\mathrm{x}$ \\
\hline
\end{tabular}

\begin{tabular}{|l||}
\hline Fees \\
\hline \hline No fees are charged. \\
\\
\hline
\end{tabular}




\section{Indiana}

\begin{tabular}{|l|l||}
\hline \multicolumn{2}{|c|}{ Grain Moisture Testing } \\
\hline \hline Address & Telephone Number \\
\hline \hline Commission on Agriculture & (317) 232-1356 \\
\hline Person responsible for program & \\
\hline Contact for additional information & \\
\hline Additional accreditation/recognition & \\
\hline
\end{tabular}

\begin{tabular}{|l|l||}
\hline \multicolumn{2}{|c|}{ Petroleum Quality Testing } \\
\hline \hline Address & Telephone Number \\
\hline \hline $\begin{array}{l}\text { Indiana State Board of Health } \\
\text { Division of Weights and Measures } \\
1330 \text { W Michigan St } \\
\text { Indianapolis, IN 46206 }\end{array}$ & $(317) 633-0350$ \\
\hline $\begin{array}{l}\text { Person responsible for program } \\
\text { Contact for additional information }\end{array}$ & Sharon Rhoades \\
\hline Additional accreditation/recognition & contracting tests \\
\hline
\end{tabular}

\begin{tabular}{|l|l||}
\hline \multicolumn{2}{|c|}{ Additional Tests or Laboratory Functions } \\
\hline \hline Address & Telephone Number \\
\hline & \\
\hline Person responsible for program & \\
\hline Contact for additional information & \\
\hline Additional accreditation/recognition & \\
\hline
\end{tabular}




\section{Iowa}

Accreditation Period: 1993

\begin{tabular}{||l|l||l||}
\hline Laboratory Staff & Address & Telephone Number \\
\hline Robert E. Hollis, Metrologist & $\begin{array}{l}\text { IA Dept of Agriculture } \\
\text { Weights and Measures Division } \\
\text { Henry Wallace Bldg } \\
\text { Des Moines, IA 50319 }\end{array}$ & FAX: (515) 281-6800 \\
\hline
\end{tabular}

\begin{tabular}{|c|c|c|c|c|c|}
\hline \multicolumn{6}{|c|}{ Services Available } \\
\hline \multicolumn{3}{|c|}{ Tolerance Testing } & \multicolumn{3}{|c|}{ Calibration } \\
\hline \multirow[t]{2}{*}{ Mass } & Weight $\leq 1000 \mathrm{lb}$ & $\mathbf{F}$ & \multirow[t]{2}{*}{ Mass } & Weight $\leq 3 \mathrm{~kg}$ & $\mathrm{x}$ \\
\hline & Weight $>1000 \mathrm{lb}$ & $\mathbf{F}$ & & Weight $>3 \mathrm{~kg}$ & $\mathrm{X}$ \\
\hline \multirow[t]{3}{*}{ Volume } & Small $\leq 25$ gal & $\mathrm{F}$ & \multirow[t]{3}{*}{ Volume } & Metal Standards & $\mathbf{F}$ \\
\hline & Intermediate $25-500 \mathrm{gal}$ & $\mathbf{F}$ & & \multirow[t]{2}{*}{ Small Glassware } & \multirow[t]{2}{*}{$\mathbf{F}$} \\
\hline & Large > $500 \mathrm{gal}$ & F & & & \\
\hline \multirow{4}{*}{$\begin{array}{l}\text { O: } \\
\text { F: } \\
\text { N: } \\
X:\end{array}$} & \multirow{4}{*}{\multicolumn{2}{|c|}{$\begin{array}{l}\text { Service to parent organization only; } \\
\text { Service available on a fee basis; } \\
\text { Service available, no fee; } \\
\text { No accreditation, contact laboratory for availability. }\end{array}$}} & \multirow[t]{2}{*}{ Length } & Steel Tapes & $\mathrm{F}$ \\
\hline & & & & Rigid Rules & $\mathbf{F}$ \\
\hline & & & Frequency & Tuning Forks & $\mathrm{X}$ \\
\hline & & & Temperature & Liquid in Glass Thermometry & $\mathrm{X}$ \\
\hline
\end{tabular}

\section{Fees}

Mass calibration, refined fuel and LPG prover calibration $\$ 10$ per hour, one hour minimum charge.

Tolerance testing mass: $\leq 5 \mathrm{lb}: \$ 2 ; \quad 5 \mathrm{lb}<\mathrm{W} \leq 50 \mathrm{lb}: \$ 5 ; \quad 50 \mathrm{lb}<\mathrm{W} \leq 100 \mathrm{lb}: \$ 8 ; \quad 100 \mathrm{lb}<\mathrm{W} \leq 500 \mathrm{lb}: \$ 10$; $500 \mathrm{lb}<\mathrm{W} \leq 1000$ : $\$ 151000 \mathrm{lb}<\mathrm{W} \leq 2500 \mathrm{lb}$ : $\$ 25$; Weight kits with 10 or more weights: $\$ 30$ per kit.

Volume measures: Laboratory glassware: \$10; Metal, 1 gal - 5 gal: \$10; Metal, 5 gal - 10 gal: \$15;

Metal, $10 \mathrm{gal}$ - 20 gal: $\$ 20$.

Linear measures: Rigid rulers: $\$ 20 ; \quad$ Steel and Fabric measuring tapes: $\$ .10 / \mathrm{ft}$ for first 10 feet, $\$ .50 / \mathrm{ft}$ for each $10 \mathrm{ft}$ interval thereafter. 


\section{Iowa}

\begin{tabular}{||l|l||}
\hline \multicolumn{2}{|c||}{ Grain Moisture Testing } \\
\hline \hline Address & Telephone Number \\
\hline $\begin{array}{l}\text { LA Dept of Agriculture } \\
\text { Weights and Measures Division } \\
\text { Henry Wallace Bldg } \\
\text { Des Moines, IA 50319 }\end{array}$ & $\begin{array}{l}(515) \text { 281-5716 } \\
(515) \text { 281-5861 }\end{array}$ \\
\hline $\begin{array}{l}\text { Person responsible for program } \\
\text { Contact for additional information }\end{array}$ & Jerry L. Bane, Bureau Chief \\
\hline Additional accreditation/recognition & Thomas Matthews, Laboratory Asst 3 \\
\hline
\end{tabular}

\begin{tabular}{|l|l||}
\hline \multicolumn{2}{|c||}{ Petroleum Quality Testing } \\
\hline \hline Address & Telephone Number \\
\hline $\begin{array}{l}\text { LA Dept of Agriculture } \\
\text { Weights and Measures Division } \\
\text { Henry Wallace Bldg } \\
\text { Des Moines, LA 50319 }\end{array}$ & $\begin{array}{l}(515) \text { 281-5716 } \\
(515) \text { 281-5861 }\end{array}$ \\
\hline Person responsible for program & Jerry L. Bane, Bureau Chief \\
\hline Contact for additional information & Robert E. Hollis, Metrologist \\
\hline Additional accreditation/recognition & \\
\hline
\end{tabular}

\begin{tabular}{|l|l||}
\hline \multicolumn{2}{|c||}{ Additional Tests or Laboratory Functions } \\
\hline Address & Telephone Number \\
\hline \hline $\begin{array}{l}\text { LA Dept of Agriculture } \\
\text { Weights and Measures Division } \\
\text { Henry Wallace Bidg } \\
\text { Des Moines, IA 50319 }\end{array}$ & $\begin{array}{l}\text { (515) 281-5716 } \\
\text { (515) 281-5861 }\end{array}$ \\
\hline Person responsible for program & Jerry L. Bane, Bureau Chief \\
\hline Contact for additional information & Robert E. Hollis, Metrologist \\
\hline Additional accreditation/recognition & \\
\hline
\end{tabular}




\section{Kansas}

Accreditation Period: 1991-1993

\begin{tabular}{||l|l|l||}
\hline \hline Laboratory Staff & Address & Telephone Number \\
\hline \hline Karl A. Herken, State Metrologist & \multicolumn{1}{|c|}{$\begin{array}{l}\text { Kansas State Board of Agriculture } \\
\text { Carl W. Gile, Metrologist } \\
\text { Weights and Measures Laboratory } \\
\text { 2016 SW 37th St } \\
\text { Topeka, KS 66611-2570 }\end{array}$} & FAX (913) 296-0673 \\
\hline
\end{tabular}

\begin{tabular}{|c|c|c|c|c|c|}
\hline \multicolumn{6}{|c|}{ Services Available } \\
\hline \multicolumn{3}{|c|}{ Tolerance Testing } & \multicolumn{3}{|c|}{ Calibration } \\
\hline \multirow[t]{2}{*}{ Mass } & Weight $\leq 1000 \mathrm{lb}$ & $\mathbf{F}$ & \multirow[t]{2}{*}{ Mass } & Weight $\leq 3 \mathrm{~kg}$ & F \\
\hline & Weight $>1000 \mathrm{lb}$ & $\mathbf{F}$ & & Weight $>3 \mathrm{~kg}$ & $\mathbf{F}$ \\
\hline \multirow[t]{3}{*}{ Volume } & Small $\leq 25$ gal & $\mathbf{F}$ & \multirow[t]{3}{*}{ Volume } & Metal Standards & $\mathbf{F}$ \\
\hline & Intermediate $25-500 \mathrm{gal}$ & $\mathbf{F}$ & & \multirow[t]{2}{*}{ Small Glassware } & \multirow[t]{2}{*}{$\mathbf{F}$} \\
\hline & Large > 500 gal & F & & & \\
\hline \multirow{4}{*}{$\begin{array}{l}\text { O: } \\
\mathrm{F}: \\
\mathrm{N}: \\
\mathrm{X}:\end{array}$} & \multirow{4}{*}{\multicolumn{2}{|c|}{$\begin{array}{l}\text { Service to parent organization only; } \\
\text { Service available on a fee basis; } \\
\text { Service available, no fee; } \\
\text { No accreditation, contact laboratory for availability. }\end{array}$}} & \multirow[t]{2}{*}{ Length } & Steel Tapes & $\mathbf{F}$ \\
\hline & & & & Rigid Rules & $\mathbf{F}$ \\
\hline & & & Frequency & Tuning Forks & $\mathrm{X}$ \\
\hline & & & Temperature & Liquid in Glass Thermometry & $\mathrm{X}$ \\
\hline
\end{tabular}

\section{Fees}

Labor...\$30 per hour. $1 / 2$ hour minimum.

\section{Remarks:}

The Kansas laboratory maintains computerized control charts for all weights used in mass calibrations as part of the measurement control program, offers control chart formulation and evaluation consulting, as well as QA program assistance to their customers. The laboratory also offers twine testing with a Scotts Tester. 
Kansas

\begin{tabular}{|l|l||}
\hline \multicolumn{2}{|c|}{ Grain Moisture Testing } \\
\hline \hline Address & Telephone Number \\
\hline $\begin{array}{l}\text { Kansas Grain Inspection } \\
700 \text { Jackson, Suite } 800 \\
\text { Box } 1918 \\
\text { Topeka, KS } 66601\end{array}$ & $(913)$ 296-3451 \\
\hline Person responsible for program & \\
\hline Contact for additional information & Gary Bothwell \\
\hline Additional accreditation/recognition & \\
\hline
\end{tabular}

\begin{tabular}{|l|l|}
\hline \multicolumn{2}{|c|}{ Petroleum Quality Testing } \\
\hline \hline Address & Telephone Number \\
\hline $\begin{array}{l}\text { Kansas Weights and Measures } \\
\text { 2016 SW 37th St } \\
\text { Topeka, KS 66611-2570 }\end{array}$ & (913) 267-4641 \\
\hline Person responsible for program & DeVern H. Phillips \\
\hline Contact for additional information & Lewis J. Hutfles \\
\hline Additional accreditation/recognition & \\
\hline
\end{tabular}

\begin{tabular}{|l|l||}
\hline \multicolumn{2}{|c|}{ National Type Evaluation Program (NTEP) } \\
\hline \hline Address & Telephone Number \\
\hline $\begin{array}{l}\text { Kansas Weights and Measures } \\
\text { 2016 SW 37th St } \\
\text { Topeka, KS 66611-2570 }\end{array}$ & $(913) 267-4641$ \\
\hline Person responsible for program & \\
\hline Contact for additional information & \\
\hline Additional accreditation/recognition & NIST Authorized Participating Laboratory \\
\hline NTEP performance testing of vehicle, axle-load, livestock, and floor scales. \\
\hline
\end{tabular}




\section{Kentucky}

Accreditation Period: 1993-1994

\begin{tabular}{||l||l|l||}
\hline \hline Laboratory Staff & Address & Telephone Number \\
\hline \hline $\begin{array}{l}\text { Randall Wise, Metrologist } \\
\begin{array}{l}\text { Leslie Hageman, Agricultural Laboratory } \\
\text { Technician }\end{array}\end{array}$ & $\begin{array}{l}\text { Division of Weights and Measures } \\
\text { 106 W 2nd St } \\
\text { Frankfort, KY 40601 }\end{array}$ & FAX: (502) 564-5669 \\
& & \\
& & \\
\hline
\end{tabular}

\begin{tabular}{|c|c|c|c|c|c|}
\hline \multicolumn{6}{|c|}{ Services Available } \\
\hline \multicolumn{3}{|c|}{ Tolerance Testing } & \multicolumn{3}{|c|}{ Calibration } \\
\hline \multirow[t]{2}{*}{ Mass } & Weight $\leq 1000 \mathrm{lb}$ & $\mathrm{N}$ & \multirow[t]{2}{*}{ Mass } & Weight $\leq 3 \mathrm{~kg}$ & $\mathrm{x}$ \\
\hline & Weight $>1000 \mathrm{lb}$ & $\mathrm{x}$ & & Weight $>3 \mathrm{~kg}$ & $\mathrm{x}$ \\
\hline \multirow[t]{3}{*}{ Volume } & Small $\leq 25$ gal & $\mathrm{N}$ & \multirow[t]{3}{*}{ Volume } & Metal Standards & $\mathrm{X}$ \\
\hline & Intermediate $25-500 \mathrm{gal}$ & $\mathrm{N}$ & & \multirow[t]{2}{*}{ Small Glassware } & \multirow[t]{2}{*}{$\mathrm{x}$} \\
\hline & Large > 500 gal & $\mathrm{N}$ & & & \\
\hline \multirow{4}{*}{$\begin{array}{l}\text { O: } \\
\text { F: } \\
\text { N: } \\
\text { X: }\end{array}$} & \multirow{4}{*}{\multicolumn{2}{|c|}{$\begin{array}{l}\text { Service to parent organization only; } \\
\text { Service available on a fee basis; } \\
\text { Service available, no fee; } \\
\text { No accreditation, contact laboratory for availability. }\end{array}$}} & \multirow[t]{2}{*}{ Length } & Steel Tapes & $\mathrm{x}$ \\
\hline & & & & Rigid Rules & $\mathrm{X}$ \\
\hline & & & Frequency & Tuning Forks & $\mathrm{x}$ \\
\hline & & & Temperature & Liquid in Glass Thermometry & $\mathrm{x}$ \\
\hline
\end{tabular}

\section{Fees}

No fees are charged. 


\section{Kentucky}

\begin{tabular}{|l|l||}
\hline \multicolumn{2}{|c|}{ Grain Moisture Testing } \\
\hline \hline Address & Telephone Number \\
\hline $\begin{array}{l}\text { Division of Weights and Measures } \\
106 \mathrm{~W} 2 \mathrm{nd} \mathrm{St} \\
\text { Frankfort, KY } 40601\end{array}$ & \\
\hline Person responsible for program $564-4870$ \\
\hline Contact for additional information & Randall Wise \\
\hline Additional accreditation/recognition & \\
\hline
\end{tabular}

\begin{tabular}{||l|l||}
\hline \multicolumn{2}{|c||}{ Petroleum Quality Testing } \\
\hline \hline Address & Telephone Number \\
\hline \hline NOT AVAILABLE & \\
\hline Person responsible for program & \\
\hline Contact for additional information & \\
\hline Additional accreditation/recognition & \\
\hline
\end{tabular}

\begin{tabular}{||l|l||}
\hline \multicolumn{2}{|c||}{ Additional Tests or Laboratory Functions } \\
\hline \hline Address & Telephone Number \\
\hline \hline & \\
\hline Person responsible for program & \\
\hline Contact for additional information & \\
\hline Additional accreditation/recognition & \\
\hline
\end{tabular}




\section{Los Angeles County}

Accreditation Period: 1993

\begin{tabular}{||l|l||l|}
\hline Laboratory Staff & Address & Telephone Number \\
\hline \hline William D. Moore, Metrologist & & $(310) 940-8931$ \\
Emil Hazarian, Metrologist & Metrology Laboratory & 11012 Garfield Ave \\
Herb Lehman & South Gate, CA 90280-7598 & FAX: (310) 861-0278 \\
K. C. Chow & & \\
& & \\
\hline
\end{tabular}

\begin{tabular}{|c|c|c|c|c|c|c|}
\hline \multicolumn{7}{|c|}{ Services Available } \\
\hline \multicolumn{4}{|c|}{ Tolerance Testing } & \multicolumn{3}{|c|}{ Calibration } \\
\hline \multirow{2}{*}{\multicolumn{2}{|c|}{ Mass }} & Weight $\leq 1000 \mathrm{lb}$ & $\mathbf{F}$ & \multirow[t]{2}{*}{ Mass } & Weight $\leq 3 \mathrm{~kg}$ & $\mathbf{F}$ \\
\hline & & Weight $>1000 \mathrm{lb}$ & $\mathrm{F}$ & & Weight > $3 \mathrm{~kg}$ & F \\
\hline \multirow{3}{*}{\multicolumn{2}{|c|}{ Volume }} & Small $\leq 25$ gal & $\mathrm{F}$ & \multirow[t]{3}{*}{ Volume } & Metal Standards & $\mathrm{F}$ \\
\hline & & Intermediate $25-500 \mathrm{gal}$ & $\mathrm{F}$ & & \multirow[t]{2}{*}{ Small Glassware } & \multirow[t]{2}{*}{$\mathrm{F}$} \\
\hline & & Large > $500 \mathrm{gal}$ & $\mathbf{F}$ & & & \\
\hline \multirow{4}{*}{$\begin{array}{l}\text { O: } \\
\text { F: } \\
\text { N: } \\
\text { X: }\end{array}$} & \multirow{4}{*}{\multicolumn{3}{|c|}{$\begin{array}{l}\text { Service to parent organization only; } \\
\text { Service available on a fee basis; } \\
\text { Service available, no fee; } \\
\text { No certification, contact laboratory for availability. }\end{array}$}} & \multirow[t]{2}{*}{ Length } & Steel Tapes & $\mathrm{O}$ \\
\hline & & & & & Rigid Rules & $\mathrm{O}$ \\
\hline & & & & Frequency & Tuning Forks & $\mathrm{x}$ \\
\hline & & & & Temperature & Liquid in Glass Thermometry & $\mathrm{x}$ \\
\hline
\end{tabular}

\section{Fees}

$\$ 45.87$ per hour, subject to change

\section{Remarks:}

The Los Angeles County laboratory has participated in the NIST Mass MAP program since 1972.

\section{Other Tests:}

None 



\section{Louisiana}

Accreditation Period: 1992-1994

\begin{tabular}{||l|l|l||}
\hline \hline Laboratory Staff & Address & Telephone Number \\
\hline \hline Pat Chaney, Metrologist & \multicolumn{1}{|l|}{$\begin{array}{l}\text { LA Dept of Agriculture and Forestry } \\
\text { Metrology Laboratory } \\
\text { PO Box 3098 } \\
\text { Baton Rouge, LA 70821-3098 }\end{array}$} & $\begin{array}{l}\text { (504) 922-1380 Lab } \\
\text { FAX: (504) 922-1289 }\end{array}$ \\
& $\begin{array}{l}\text { Shipping: 5825 Florida Blvd } \\
\text { Baton Rouge, LA 70806 }\end{array}$ & \\
\hline
\end{tabular}

\begin{tabular}{|c|c|c|c|c|c|}
\hline \multicolumn{6}{|c|}{ Services Available } \\
\hline \multicolumn{3}{|c|}{ Tolerance Testing } & \multicolumn{3}{|c|}{ Calibration } \\
\hline \multirow[t]{2}{*}{ Mass } & Weight $\leq 1000 \mathrm{lb}$ & F & \multirow[t]{2}{*}{ Mass } & Weight $\leq 3 \mathrm{~kg}$ & $\mathrm{~F}$ \\
\hline & Weight $>1000 \mathrm{lb}$ & $\mathbf{F}$ & & Weight $>3 \mathrm{~kg}$ & $\mathbf{F}$ \\
\hline \multirow[t]{3}{*}{ Volume } & Small $\leq 25$ gal & F & \multirow[t]{3}{*}{ Volume } & Metal Standards & $\mathrm{F}$ \\
\hline & Intermediate $25-500 \mathrm{gal}$ & $\mathrm{X}$ & & Small Glassware & $\mathrm{F}$ \\
\hline & Large > $500 \mathrm{gal}$ & $\mathrm{X}$ & & & \\
\hline \multirow{4}{*}{$\begin{array}{l}\text { O: } \\
\text { F: } \\
\text { N: } \\
X:\end{array}$} & \multirow{4}{*}{\multicolumn{2}{|c|}{$\begin{array}{l}\text { Service to parent organization only; } \\
\text { Service available on a fee basis; } \\
\text { Service available, no fee; } \\
\text { No accreditation, contact laboratory for availability. }\end{array}$}} & \multirow[t]{2}{*}{ Length } & Steel Tapes & $\mathbf{F}$ \\
\hline & & & & Rigid Rules & $\mathrm{F}$ \\
\hline & & & Frequency & Tuning Forks & $\mathrm{x}$ \\
\hline & & & Temperature & Liquid in Glass Thermometry & $\mathrm{X}$ \\
\hline
\end{tabular}

\section{Fees}

Labor... Length and Volume: $\$ 25$ per hour. Minimum one hour charge.

Mass - Tolerance Testing: $W \leq 10 \mathrm{lb}: \$ 2 ; \mathrm{W} \leq 100 \mathrm{lb}: \$ 5 ; \mathrm{W} \leq 1000 \mathrm{lb}: \$ 25 ; \mathrm{W}>1000 \mathrm{lb}: \$ 50$;

Mass - Calibration: $W \leq 3 \mathrm{~kg}: \$ 25 ; W \leq 30 \mathrm{~kg}: \$ 50 ; W>30 \mathrm{~kg}: \$ 75$

\section{Remarks:}

Louisiana moved into a new laboratory in 1989. 


\section{Louisiana}

\begin{tabular}{||l|l||}
\hline \multicolumn{2}{|c|}{ Grain Moisture Testing } \\
\hline \hline Address & Telephone Number \\
\hline \hline NOT AVAILABLE & \\
\hline Person responsible for program & \\
\hline Contact for additional information & \\
\hline Additional accreditation/recognition & \\
\hline
\end{tabular}

\begin{tabular}{||l|l||}
\hline \multicolumn{2}{|c|}{ Petroleum Quality Testing } \\
\hline \hline Address & Telephone Number \\
\hline NOT AVAILABLE & \\
& \\
\hline Person responsible for program & \\
\hline Contact for additional information & \\
\hline Additional accreditation/recognition & \\
\hline
\end{tabular}

\begin{tabular}{|l|l||}
\hline \multicolumn{2}{|c|}{ Additional Tests or Laboratory Functions } \\
\hline \hline Address & Telephone Number \\
\hline \hline NOT AVAILABLE & \\
\hline Person responsible for program & \\
\hline Contact for additional information & \\
\hline Additional accreditation/recognition & \\
\hline
\end{tabular}




\section{Maine}

Accreditation Period: 1991-1993

\begin{tabular}{||l|l|l||}
\hline Laboratory Staff & Address & Telephone Number \\
\hline Stanley K. Millay, Metrology Supervisor & $\begin{array}{l}\text { ME Dept of Agriculture } \\
\text { Division of Regulations } \\
\text { Station 28 } \\
\text { Augusta, ME 04333 }\end{array}$ & FAX: (207) 287-7161 \\
\hline
\end{tabular}

\begin{tabular}{|c|c|c|c|c|c|}
\hline \multicolumn{6}{|c|}{ Services Available } \\
\hline \multicolumn{3}{|c|}{ Tolerance Testing } & \multicolumn{3}{|c|}{ Calibration } \\
\hline \multirow[t]{2}{*}{ Mass } & Weight $\leq 1000 \mathrm{lb}$ & $\mathbf{F}$ & \multirow[t]{2}{*}{ Mass } & Weight $\leq 3 \mathrm{~kg}$ & $\mathbf{F}$ \\
\hline & Weight > $1000 \mathrm{lb}$ & $\mathrm{x}$ & & Weight $>3 \mathrm{~kg}$ & $\mathbf{F}$ \\
\hline \multirow[t]{3}{*}{ Volume } & Small $\leq 25$ gal & F & \multirow[t]{3}{*}{ Volume } & Metal Standards & F \\
\hline & Intermediate 25 - 500 gal & F & & \multirow[t]{2}{*}{ Small Glassware } & \multirow[t]{2}{*}{$\mathrm{F}$} \\
\hline & Large $>500 \mathrm{gal}$ & $\mathrm{F}$ & & & \\
\hline \multirow{4}{*}{$\begin{array}{l}\mathrm{O}: \\
\mathrm{F}: \\
\mathrm{N}: \\
\mathrm{X}:\end{array}$} & \multirow{4}{*}{\multicolumn{2}{|c|}{$\begin{array}{l}\text { Service to parent organization only, } \\
\text { Service available on a fee basis; } \\
\text { Service available, no fee; } \\
\text { No accreditation, contact laboratory for availability. }\end{array}$}} & \multirow[t]{2}{*}{ Length } & Steel Tapes & F \\
\hline & & & & Rigid Rules & $\mathbf{F}$ \\
\hline & & & Frequency & Tuning Forks & F \\
\hline & & & Temperature & Liquid in Glass Thermometry & $\mathrm{x}$ \\
\hline
\end{tabular}

\section{Fees}

Labor...\$40 per hour.

\section{Remarks:}

Maine has an indoor, drive-through, truck compartment volume calibration facility. Maine also provides tolerance testing of stopwatches and calibration of Sardine Carrier holds. 
Maine

\begin{tabular}{||l|l||}
\hline \multicolumn{2}{|c|}{ Grain Moisture Testing } \\
\hline \hline Address & Telephone Number \\
\hline $\begin{array}{l}\text { ME Dept of Agriculture } \\
\text { Division of Regulations } \\
\text { Station 28 } \\
\text { Augusta, ME 04333 }\end{array}$ & $(207)$ 289-7550 \\
\hline Person responsible for program & \\
\hline Contact for additional information & Reginal Andrews \\
\hline Additional accreditation/recognition & \\
\hline
\end{tabular}

\begin{tabular}{||l|l||}
\hline \multicolumn{2}{|c|}{ Petroleum Quality Testing } \\
\hline \hline Address & Telephone Number \\
\hline \hline $\begin{array}{l}\text { ME Dept of Agriculture } \\
\text { Division of Regulations } \\
\text { Station 28 } \\
\text { Augusta, ME 04333 }\end{array}$ & (207) 289-3841 \\
\hline Person responsible for program & Stanley K. Millay \\
\hline Contact for additional information & \\
\hline Additional accreditation/recognition & \\
\hline
\end{tabular}

\begin{tabular}{|l|l||}
\hline \multicolumn{2}{|c|}{ Additional Tests or Laboratory Functions } \\
\hline \hline Address & Telephone Number \\
\hline & \\
\hline Person responsible for program & \\
\hline Contact for additional information & \\
\hline Additional accreditation/recognition & \\
\hline \hline
\end{tabular}




\section{Maryland}

Accreditation Period: 1993-1995

\begin{tabular}{||l|l|l||}
\hline \hline Laboratory Staff & Address & Telephone Number \\
\hline \hline $\begin{array}{l}\text { Charles R. Stockman, Lab Program Manager } \\
\text { Steve Barry, Metrology Technician }\end{array}$ & $\begin{array}{l}\text { MD Dept of Agriculture } \\
\text { 50 Harry S Truman Pkwy } \\
\text { Annapolis, MD 21401 }\end{array}$ & FAX: (410) 841-2765 \\
$\begin{array}{l}\text { Victor P. DeLibera, Metrology Technician } \\
\text { Gloria Diane Lee/London, Metrology } \\
\text { Technician }\end{array}$ & & \\
& & \\
\hline
\end{tabular}

\begin{tabular}{|c|c|c|c|c|c|}
\hline \multicolumn{6}{|c|}{ Services Available } \\
\hline \multicolumn{3}{|c|}{ Tolerance Testing } & \multicolumn{3}{|c|}{ Calibration } \\
\hline \multirow[t]{2}{*}{ Mass } & Weight $\leq 1000 \mathrm{lb}$ & $\mathrm{F}$ & \multirow[t]{2}{*}{ Mass } & Weight $\leq 3 \mathrm{~kg}$ & $\mathrm{~F}$ \\
\hline & Weight $>1000 \mathrm{lb}$ (up to $20,000 \mathrm{lb}$ ) & F & & Weight $>3 \mathrm{~kg}$ (up to $20,000 \mathrm{lb}$ ) & $\mathrm{F}$ \\
\hline \multirow[t]{3}{*}{ Volume } & Small $\leq 25$ gal & F & \multirow[t]{3}{*}{ Volume } & Metal Standards & $\mathrm{F}$ \\
\hline & Intermediate 25 - $500 \mathrm{gal}$ & $\mathrm{F}$ & & \multirow[t]{2}{*}{ Small Glassware } & \multirow[t]{2}{*}{$\mathrm{F}$} \\
\hline & Large > 500 gal & $\mathbf{F}$ & & & \\
\hline \multirow{4}{*}{$\begin{array}{l}\mathrm{O}: \\
\mathrm{F}: \\
\mathrm{N}: \\
\mathrm{X}:\end{array}$} & \multirow{4}{*}{\multicolumn{2}{|c|}{$\begin{array}{l}\text { Service to parent organization only; } \\
\text { Service available on a fee basis; } \\
\text { Service available, no fee; } \\
\text { No accreditation, contact laboratory for availability. }\end{array}$}} & \multirow[t]{2}{*}{ Length } & Steel Tapes & $\mathrm{F}$ \\
\hline & & & & Rigid Rules & $\mathrm{F}$ \\
\hline & & & Frequency & Tuning Forks & $\mathrm{x}$ \\
\hline & & & Temperature & Liquid in Glass Thermometry & F \\
\hline
\end{tabular}

\section{Fees}

Labor...S45 per hour. Contact laboratory for additional capabilities and fee schedule.

Refurbishing of measures/standards is available. Contact laboratory for specifics.

\section{Remarks:}

Maryland moved into a new laboratory in August, 1990. Laboratory tests stopwatches and also dial gauges which are used to test polyethyiene sheeting, glassware used for Babcock tests of milk, vapor meters, and slow-flow meters. 
Maryland

\begin{tabular}{|l|l||}
\hline \multicolumn{2}{|c|}{ Grain Moisture Testing } \\
\hline \hline Address & Telephone Number \\
\hline $\begin{array}{l}\text { MD Dept of Agriculture } \\
\text { 50 Harry S Truman Pkwy } \\
\text { Annapolis, MD 21401 }\end{array}$ & $(410)$ 841-5790 \\
\hline Person responsible for program & \\
\hline Contact for additional information & Gloria Diane Lee/London \\
\hline Additional accreditation/recognition & \\
\hline
\end{tabular}

\begin{tabular}{|l|l||}
\hline \multicolumn{2}{|c||}{ Petroleum Quality Testing } \\
\hline \hline Address & Telephone Number \\
\hline \hline $\begin{array}{l}\text { State of Maryland } \\
\text { Motor Fuel Testing Laboratory } \\
7101 \text { Jessup Rd } \\
\text { Jessup, MD 20794 }\end{array}$ & $\begin{array}{l}(410) 799-7777 \\
(410) 974-3126\end{array}$ \\
\hline Person responsible for program & Robert Crawford \\
\hline Contact for additional information & Stephen Taylor, Michael Jordan \\
\hline Additional accreditation/recognition & \\
\hline
\end{tabular}

\begin{tabular}{||l|l||}
\hline \multicolumn{2}{|c||}{ National Type Evaluation Program (NTEP) } \\
\hline \hline Address & Telephone Number \\
\hline \hline $\begin{array}{l}\text { MD Dept of Agriculture } \\
\text { 50 Harry S Truman Pkwy } \\
\text { Annapolis, MD 21401 }\end{array}$ & $(410) 841-5790$ \\
\hline Person responsible for program & \\
\hline Contact for additional information & Constantine V. Cotsoradis \\
\hline Additional accreditation/recognition & \\
\hline NTEP testing of commercial scales and weighing devices, liquid measuring devices, and cash registers interfaced with scales. \\
\hline
\end{tabular}




\section{Massachusetts}

Accreditation Period: 1993

\begin{tabular}{||l|l|l||}
\hline \hline Laboratory Staff & Address & Telephone Number \\
\hline Donald W. Smith, Metrologist & $\begin{array}{l}\text { MA Division of Standards } \\
\text { One Ashburton Place } \\
\text { Boston, MA 02108 }\end{array}$ & \\
\hline
\end{tabular}

\begin{tabular}{|c|c|c|c|c|c|}
\hline \multicolumn{6}{|c|}{ Services Available } \\
\hline \multicolumn{3}{|c|}{ Tolerance Testing } & \multicolumn{3}{|c|}{ Calibration } \\
\hline \multirow[t]{2}{*}{ Mass } & Weight $\leq 1000 \mathrm{lb}$ & $\mathrm{F}$ & \multirow[t]{2}{*}{ Mass } & Weight $\leq 3 \mathrm{~kg}$ & $\mathrm{~F}$ \\
\hline & Weight $>1000 \mathrm{lb}$ & $\mathrm{x}$ & & Weight $>3 \mathrm{~kg}$ & $\mathbf{F}$ \\
\hline \multirow[t]{3}{*}{ Volume } & Small $\leq 25$ gal & $\mathbf{F}$ & \multirow[t]{3}{*}{ Volume } & Metal Standards & $\mathbf{F}$ \\
\hline & Intermediate $25-500 \mathrm{gal}$ & $\mathbf{F}$ & & \multirow[t]{2}{*}{ Small Glassware } & \multirow[t]{2}{*}{$\mathrm{F}$} \\
\hline & Large > 500 gal & $\mathrm{x}$ & & & \\
\hline \multirow{4}{*}{$\begin{array}{l}\text { O: } \\
\text { F: } \\
\text { N: } \\
\text { X: }\end{array}$} & \multirow{4}{*}{\multicolumn{2}{|c|}{$\begin{array}{l}\text { Service to parent organization only, } \\
\text { Service available on a fee basis; } \\
\text { Service available, no fee; } \\
\text { No accreditation, contact laboratory for availability. }\end{array}$}} & \multirow[t]{2}{*}{ Length } & Steel Tapes & $\mathbf{F}$ \\
\hline & & & & Rigid Rules & F \\
\hline & & & Frequency & Tuning Forks & $\mathrm{x}$ \\
\hline & & & Temperature & Liquid in Glass Thermometry & $\mathrm{x}$ \\
\hline
\end{tabular}

\section{Fees}

Labor...\$35 per hour.

Tolerance testing, Class F, $\$ 4$ each weight, $\$ 2$ adjustment fee. 


\section{Massachusetts}

\begin{tabular}{|l|l||}
\hline \multicolumn{2}{|c|}{ Grain Moisture Testing } \\
\hline \hline Address & Telephone Number \\
\hline \hline NOT AVAILABLE & \\
\hline Person responsible for program & \\
\hline Contact for additional information & \\
\hline Additional accreditation/recognition & \\
\hline
\end{tabular}

\begin{tabular}{|l|l|}
\hline \multicolumn{2}{|c|}{ Petroleum Quality Testing } \\
\hline Address & Telephone Number \\
\hline $\begin{array}{l}\text { Division of Standards } \\
\text { One Ashburton Place } \\
\text { Boston, MA 02108 }\end{array}$ & $(617)$ 727-3480 \\
\hline Person responsible for program & Charles H. Carroll \\
\hline Contact for additional information & \\
\hline Additional accreditation/recognition & \\
\hline
\end{tabular}

\begin{tabular}{|l|l|}
\hline \multicolumn{2}{|c|}{ Additional Tests or Laboratory Functions } \\
\hline \hline Address & Telephone Number \\
\hline \hline & \\
& \\
\hline Person responsible for program & \\
\hline Contact for additional information & \\
\hline Additional accreditation/recognition & \\
\hline
\end{tabular}




\section{Michigan}

Accreditation Period: 1993-1995

\begin{tabular}{|l|l|l||}
\hline Laboratory Staff & Address & Telephone Number \\
\hline $\begin{array}{l}\text { Harold Birgy, Metrologist/Weights and } \\
\text { Measures Specialist }\end{array}$ & $\begin{array}{l}\text { MI Dept of Agriculture } \\
\text { Food Division } \\
\text { Ottawa Bldg, 4th FI } \\
\text { PO Box 30017 } \\
\text { Lansing, MI 48909 }\end{array}$ & FAX: (517) 373-3333 \\
\hline
\end{tabular}

\begin{tabular}{|c|c|c|c|c|c|}
\hline \multicolumn{6}{|c|}{ Services Available } \\
\hline \multicolumn{3}{|c|}{ Tolerance Testing } & \multicolumn{3}{|c|}{ Calibration } \\
\hline \multirow[t]{2}{*}{ Mass } & Weight $\leq 1000 \mathrm{lb}$ & F & \multirow[t]{2}{*}{ Mass } & Weight $\leq 3 \mathrm{~kg}$ & $\mathrm{~F}$ \\
\hline & Weight $>1000 \mathrm{lb}$ up to $5000 \mathrm{lb}$ & $\mathbf{F}$ & & Weight $>3 \mathrm{~kg}$ & $\mathbf{F}$ \\
\hline \multirow[t]{3}{*}{ Volume } & Small $\leq 25$ gal & $\mathbf{F}$ & \multirow[t]{3}{*}{ Volume } & Metal Standards & $\mathbf{F}$ \\
\hline & Intermediate $25-500 \mathrm{gal}$ & $\mathrm{F}$ & & \multirow[t]{2}{*}{ Small Glassware } & \multirow[t]{2}{*}{$\mathbf{F}$} \\
\hline & Large > 500 gal & $\mathrm{F}$ & & & \\
\hline \multirow{4}{*}{$\begin{array}{l}\text { O: } \\
\text { F: } \\
\text { N: } \\
\text { X: }\end{array}$} & \multirow{4}{*}{\multicolumn{2}{|c|}{$\begin{array}{l}\text { Service to parent organization only; } \\
\text { Service available on a fee basis; } \\
\text { Service available, no fee; } \\
\text { No accreditation, contact laboratory for availability. }\end{array}$}} & \multirow[t]{2}{*}{ Length } & Steel Tapes & $\mathbf{F}$ \\
\hline & & & & Rigid Rules & $\mathbf{F}$ \\
\hline & & & Frequency & Tuning Forks & $\mathrm{X}$ \\
\hline & & & Temperature & Liquid in Glass Thermometry & $\mathrm{X}$ \\
\hline
\end{tabular}

\begin{tabular}{|l||}
\hline Fees \\
\hline Labor...\$55 per hour. \\
\hline
\end{tabular}

\section{Remarks:}

The Michigan laboratory has a 25-gallon slicker plate volume standard for precision volume calibration. 


\section{Michigan}

\begin{tabular}{||l|l||}
\hline \multicolumn{2}{|c||}{ Grain Moisture Testing } \\
\hline \hline Address & Telephone Number \\
\hline \hline $\begin{array}{l}\text { Food Division } \\
\text { Michigan Department of Agriculture } \\
\text { PO Box } 30017 \\
\text { Lansing, MI } 48909\end{array}$ & (517) 373-1060 \\
\hline Person responsible for program & \\
\hline Contact for additional information & M. J. Mosier \\
\hline Additional accreditation/recognition & E. C. Heffron \\
\hline
\end{tabular}

\begin{tabular}{|l|l||}
\hline \multicolumn{2}{|c||}{ Petroleum Quality Testing } \\
\hline \hline Address & Telephone Number \\
\hline \hline $\begin{array}{l}\text { Food Division } \\
\text { Michigan Department of Agriculture } \\
\text { PO Box 30017 } \\
\text { Lansing, MI } 48909\end{array}$ & $(517)$ 373-1060 \\
\hline Person responsible for program & Celeste Bennett \\
\hline Contact for additional information & E. C. Heffron \\
\hline Additional accreditation/recognition & \\
\hline
\end{tabular}

\begin{tabular}{||l|l||}
\hline \multicolumn{2}{|c|}{ Additional Tests or Laboratory Functions } \\
\hline \hline Address & Telephone Number \\
\hline \hline $\begin{array}{l}\text { 320 S. Walnut } \\
\text { Lansing, MI } 48909\end{array}$ & $(517) 373-9897$ \\
\hline Person responsible for program & \\
\hline Contact for additional information & Harold Birgy \\
\hline Additional accreditation/recognition & \\
\hline \hline
\end{tabular}




\section{Minnesota}

Accreditation Period: 1992-1994

\begin{tabular}{|c|c|c|}
\hline Laboratory Staff & Address & Telephone Number \\
\hline $\begin{array}{l}\text { David Dikken, Metrologist } \\
\text { Bruce A. Adams, Metrologist }\end{array}$ & $\begin{array}{l}\text { Dept of Public Service } \\
\text { Division of Weights and Measures } \\
2277 \text { Hwy } 36 \\
\text { St. Paul, MN } 55113\end{array}$ & $\begin{array}{l}(612) 639-4010 \\
\text { FAX: }(612) 639-4014\end{array}$ \\
\hline
\end{tabular}

\begin{tabular}{|c|c|c|c|c|c|}
\hline \multicolumn{6}{|c|}{ Services Available } \\
\hline \multicolumn{3}{|c|}{ Tolerance Testing } & \multicolumn{3}{|c|}{ Calibration } \\
\hline \multirow[t]{2}{*}{ Mass } & Weight $\leq 1000 \mathrm{lb}$ & $\mathrm{F}$ & \multirow[t]{2}{*}{ Mass } & Weight $\leq 3 \mathrm{~kg}$ & $\mathbf{F}$ \\
\hline & Weight $>1000 \mathrm{lb}$ & $\mathrm{F}$ & & Weight $>3 \mathrm{~kg}$ & F \\
\hline \multirow[t]{3}{*}{ Volume } & Small $\leq 25$ gal & $\mathrm{F}$ & \multirow[t]{3}{*}{ Volume } & Metal Standards & $\mathrm{F}$ \\
\hline & Intermediate $25-500 \mathrm{gal}$ & $\mathrm{F}$ & & \multirow[t]{2}{*}{ Small Glassware } & \multirow[t]{2}{*}{$\mathrm{F}$} \\
\hline & Large > 500 gal & $\mathrm{F}$ & & & \\
\hline \multirow{4}{*}{$\begin{array}{l}\text { O: } \\
\text { F: } \\
\text { N: } \\
\text { X: }\end{array}$} & \multirow{4}{*}{\multicolumn{2}{|c|}{$\begin{array}{l}\text { Service to parent organization only; } \\
\text { Service available on a fee basis; } \\
\text { Service available, no fee; } \\
\text { No accreditation, contact laboratory for availability. }\end{array}$}} & \multirow[t]{2}{*}{ Length } & Steel Tapes & $\mathrm{F}$ \\
\hline & & & & Rigid Rules & $\mathrm{F}$ \\
\hline & & & Frequency & Tuning Forks & $\mathrm{x}$ \\
\hline & & & Temperature & Liquid in Glass Thermometry & $\mathrm{F}$ \\
\hline
\end{tabular}

\begin{tabular}{||l||}
\hline \multicolumn{1}{|c|}{ Fees } \\
\hline \hline Labor...\$75 per hour. \\
\\
\end{tabular}

\section{Remarks:}

Minnesota metrologists have received NIST Mass MAP training, and calibration of precision weights is performed using weighing designs and data are reduced with NIST Mass Code computer software. 


\section{Minnesota}

\begin{tabular}{||l|l||}
\hline \multicolumn{2}{|c|}{ Grain Moisture Testing } \\
\hline \hline Address & Telephone Number \\
\hline NOT AVAILABLE & \\
\hline Person responsible for program & \\
\hline Contact for additional information & \\
\hline Additional accreditation/recognition & \\
\hline
\end{tabular}

\begin{tabular}{||l|l||}
\hline \multicolumn{2}{|c|}{ Petroleum Quality Testing } \\
\hline \hline Address & Telephone Number \\
\hline $\begin{array}{l}\text { Minnesota Department of Public Service } \\
\text { Weights and Measures Division } \\
\text { 2277 Hwy 36 } \\
\text { St. Paul, MN 55113 }\end{array}$ & (612) 639-4010 \\
\hline Person responsible for program & Michael Blacik \\
\hline Contact for additional information & Richard Johnson \\
\hline Additional accreditation/recognition & \\
\hline
\end{tabular}

\begin{tabular}{||l|l||}
\hline \multicolumn{2}{|c|}{ Additional Tests or Laboratory Functions } \\
\hline \hline Address & Telephone Number \\
\hline \begin{tabular}{l}
\hline 2277 Hwy 36 \\
St. Paul, MN 55113
\end{tabular} & $(612) 639-4010$ \\
\hline Person responsible for program & Bruce A. Adams \\
\hline Contact for additional information & \\
\hline Additional accreditation/recognition & $\begin{array}{l}\text { Railroad Test Car calibration at Minnesota Master Track } \\
\text { Scale. }\end{array}$ \\
\hline
\end{tabular}




\section{Mississippi}

Accreditation Period: 1993

\begin{tabular}{|l|l|l||}
\hline \hline Laboratory Staff & Address & Telephone Number \\
\hline \hline Kelvin Boone, Metrologist & $\begin{array}{l}\text { PO Box 1200 } \\
\text { Alcorn State University } \\
\text { Lorman, MS 39096 }\end{array}$ & (601) 877- 3802 \\
& & \\
\hline
\end{tabular}

\begin{tabular}{|c|c|c|c|c|c|}
\hline \multicolumn{6}{|c|}{ Services Available } \\
\hline \multicolumn{3}{|c|}{ Tolerance Testing } & \multicolumn{3}{|c|}{ Calibration } \\
\hline \multirow[t]{2}{*}{ Mass } & Weight $\leq 1000 \mathrm{lb}$ & $\mathrm{N}$ & \multirow[t]{2}{*}{ Mass } & Weight $\leq 3 \mathrm{~kg}$ & $\mathrm{x}$ \\
\hline & Weight $>1000 \mathrm{lb}$ & $\mathrm{x}$ & & Weight $>3 \mathrm{~kg}$ & $\mathrm{x}$ \\
\hline \multirow[t]{3}{*}{ Volume } & Small $\leq 25$ gal & $\mathrm{N}$ & \multirow[t]{3}{*}{ Volume } & Metal Standards & $\mathrm{x}$ \\
\hline & Intermediate $25-500$ ga & $\mathrm{x}$ & & \multirow[t]{2}{*}{ Small Glassware } & \multirow[t]{2}{*}{$\mathrm{x}$} \\
\hline & Large > $500 \mathrm{gal}$ & $\mathrm{x}$ & & & \\
\hline \multirow{4}{*}{$\begin{array}{l}\text { O: } \\
\text { F: } \\
\text { N: } \\
X:\end{array}$} & \multirow{4}{*}{\multicolumn{2}{|c|}{$\begin{array}{l}\text { Service to parent organization only, } \\
\text { Service available on a fee basis; } \\
\text { Service available, no fee; } \\
\text { No accreditation, contact laboratory for availability. }\end{array}$}} & \multirow[t]{2}{*}{ Length } & Steel Tapes & $\mathrm{x}$ \\
\hline & & & & Rigid Rules & $\mathrm{x}$ \\
\hline & & & Frequency & Tuning Forks & $\mathrm{x}$ \\
\hline & & & Temperature & Liquid in Glass Thermometry & $\mathrm{x}$ \\
\hline
\end{tabular}

\begin{tabular}{||l||}
\hline Fees \\
\hline No fees are charged. \\
\\
\\
\end{tabular}




\section{Mississippi}

\begin{tabular}{|l|l||}
\hline \multicolumn{2}{|c||}{ Grain Moisture Testing } \\
\hline \hline Address & Telephone Number \\
\hline $\begin{array}{l}\text { Moisture Meter Testing Laboratory } \\
\text { Delta State University } \\
\text { PO Box 3323 } \\
\text { Cleveland, MS 38733 }\end{array}$ & $\begin{array}{l}(601) 843-1078 \\
(601) 354-7077\end{array}$ \\
\hline Person responsible for program & Janice Proctor \\
\hline Contact for additional information & William Eldridge \\
\hline Additional accreditation/recognition & \\
\hline
\end{tabular}

\begin{tabular}{||l|l||}
\hline \multicolumn{2}{|c||}{ Petroleum Quality Testing } \\
\hline \hline Address & Telephone Number \\
\hline $\begin{array}{l}\text { Mississippi Department of Agriculture and Commerce } \\
\text { Petroleum Testing Divsion } \\
\text { PO Box } 1609 \\
\text { Jackson, MS 39215-1609 }\end{array}$ & $(601) 354-6202$ \\
\hline $\begin{array}{l}\text { Person responsible for program } \\
\text { Contact for additional information }\end{array}$ & Tommy Harrell \\
\hline Additional accreditation/recognition & Connie Braswell \\
\hline
\end{tabular}

\begin{tabular}{|l|l||}
\hline \multicolumn{2}{|c|}{ Additional Tests or Laboratory Functions } \\
\hline \hline Address & Telephone Number \\
\hline & \\
\hline Person responsible for program & \\
\hline Contact for additional information & \\
\hline Additional accreditation/recognition & \\
\hline
\end{tabular}




\section{Missouri}

Accreditation Period: 1993

\begin{tabular}{||l|l|l||}
\hline Laboratory Staff & Address & Telephone Number \\
\hline $\begin{array}{l}\text { Robert D. Wittenberger, Laboratory Program } \\
\text { Supervisor, Metrologist }\end{array}$ & $\begin{array}{l}\text { Mailing: MO Dept of Agriculture } \\
\text { PO Box 630 } \\
\text { Jefferson City, MO 65102 } \\
\text { Shipping: 1616 Missouri Blvd } \\
\text { Jefferson City, MO 65109 }\end{array}$ & \\
& & \\
\hline
\end{tabular}

\begin{tabular}{|c|c|c|c|c|c|}
\hline \multicolumn{6}{|c|}{ Services Available } \\
\hline \multicolumn{3}{|c|}{ Tolerance Testing } & \multicolumn{3}{|c|}{ Calibration } \\
\hline \multirow[t]{2}{*}{ Mass } & Weight $\leq 1000 \mathrm{lb}$ & $\mathrm{F}$ & \multirow[t]{2}{*}{ Mass } & Weight $\leq 3 \mathrm{~kg}$ & $\mathbf{F}$ \\
\hline & Weight > $1000 \mathrm{lb}$ & $\mathrm{F}$ & & Weight $>3 \mathrm{~kg}$ & $\mathbf{F}$ \\
\hline \multirow[t]{3}{*}{ Volume } & Small $\leq 25$ gal & $\mathrm{F}$ & \multirow[t]{3}{*}{ Volume } & Metal Standards & $\mathrm{O}$ \\
\hline & Intermediate $25-500 \mathrm{gal}$ & $\mathrm{F}$ & & \multirow[t]{2}{*}{ Small Glassware } & \multirow[t]{2}{*}{$\mathrm{O}$} \\
\hline & Large $>500$ gal & $\mathbf{F}$ & & & \\
\hline \multirow{4}{*}{$\begin{array}{l}\text { O: } \\
\text { F: } \\
\text { N: } \\
\text { X: }\end{array}$} & \multirow{4}{*}{\multicolumn{2}{|c|}{$\begin{array}{l}\text { Service to parent organization only; } \\
\text { Service available on a fee basis; } \\
\text { Service available, no fee; } \\
\text { No accreditation, contact laboratory for availability. }\end{array}$}} & \multirow[t]{2}{*}{ Length } & Steel Tapes & $\mathrm{O}$ \\
\hline & & & & Rigid Rules & $\mathrm{O}$ \\
\hline & & & Frequency & Tuning Forks & $\mathrm{O}$ \\
\hline & & & Temperature & Liquid in Glass Thermometry & $\mathrm{X}$ \\
\hline
\end{tabular}

\section{Fees}

Labor...\$25 per hour, tolerance testing; $\$ 35$ per hour, calibration. One hour minimum charge, time over one hour rounded to the nearest hour.

\section{Remarks:}

Missouri moved into a new laboratory in 1986. 


\section{Missouri}

\begin{tabular}{|l|l||}
\hline \multicolumn{2}{|c|}{ Grain Moisture Testing } \\
\hline \hline Address & Telephone Number \\
\hline $\begin{array}{l}\text { 1616 Missouri Blvd } \\
\text { Jefferson City, NiO } 65109\end{array}$ & (314) 751-3440 \\
\hline Person responsible for program & Robert D. Wittenberger \\
\hline Contact for ađditional information & \\
\hline Additional accreditation/recognition & \\
\hline
\end{tabular}

\begin{tabular}{|l|l||}
\hline \multicolumn{2}{|c||}{ Petroleum Quality Testing } \\
\hline \hline Address & Telephone Number \\
\hline \hline $\begin{array}{l}1620 \text { Missouri Blvd } \\
\text { Jefferson City, MO } 65109\end{array}$ & (314) 751-2922 \\
\hline Person responsible for program & Ronald G. Hayes \\
\hline Contact for additional information & Carolyn Kempker \\
\hline Additional accreditation/recognition & ASTM \\
\hline
\end{tabular}

\begin{tabular}{||l|l||}
\hline \multicolumn{2}{|c|}{ Additional Tests or Laboratory Functions } \\
\hline \hline Address & Telephone Number \\
\hline \hline & \\
\hline Person responsible for program & \\
\hline Contact for additional information & \\
\hline Additional accreditation/recognition & \\
\hline
\end{tabular}




\section{Montana}

Accreditation Period: 1993

\begin{tabular}{||l|l|l||}
\hline \hline Laboratory Staff & Address & Telephone Number \\
\hline \hline Dan Nisbet, Metrologist & & (406) 449-2582 \\
& Bureau of Weights and Measures & \\
& 1424 E 9th Ave & \\
& Helena, MT 59620-0423 & \\
& & \\
& & \\
\hline
\end{tabular}

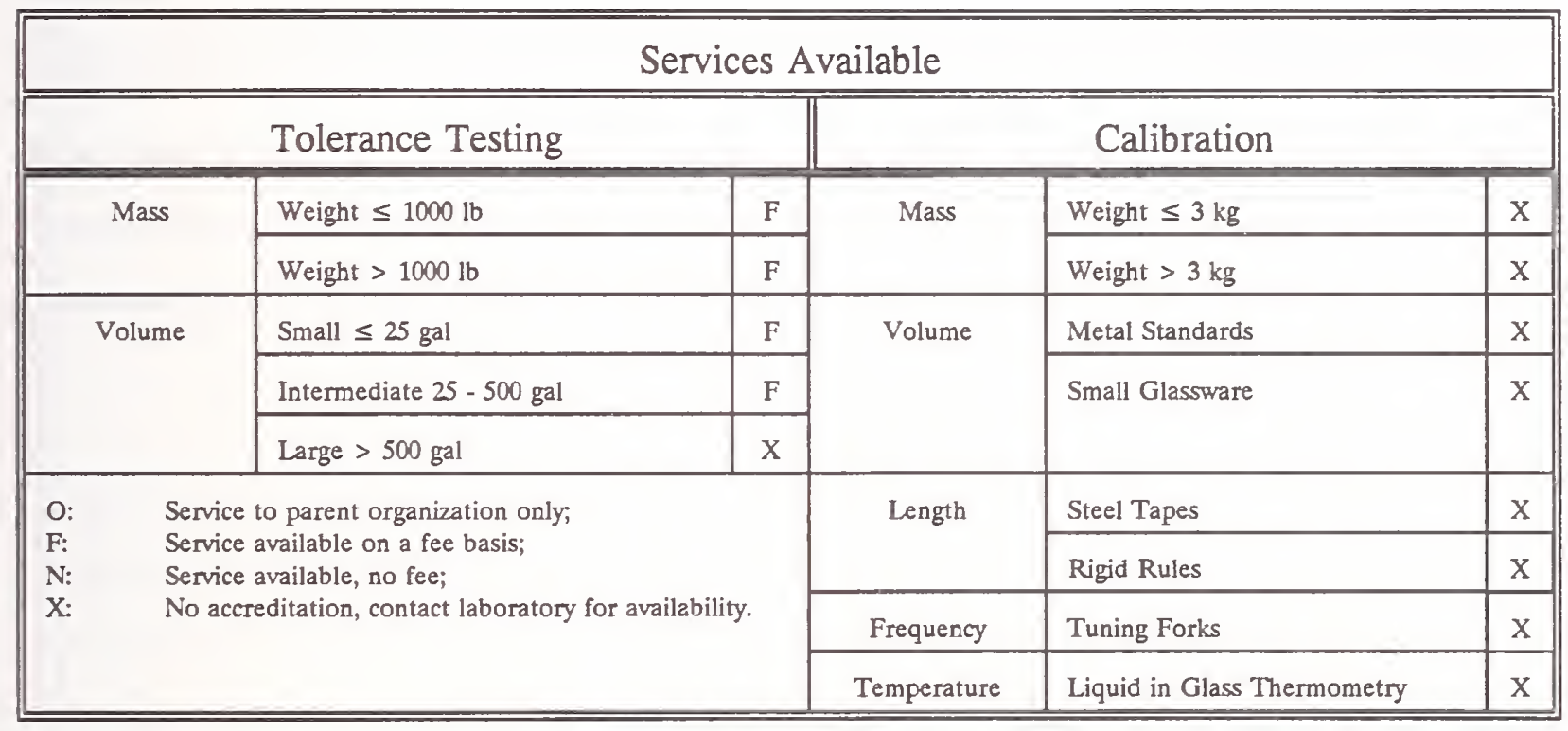

\begin{tabular}{|l||}
\hline Fees \\
\hline Labor...\$20 per hour. \\
\\
\end{tabular}

\section{Remarks:}

Laboratory is not operated on a full-time basis. 


\section{Montana}

\begin{tabular}{|l|l||}
\hline \multicolumn{2}{|c|}{ Grain Moisture Testing } \\
\hline \hline Address & Telephone Number \\
\hline NOT AVAILABLE & \\
\hline Person responsible for program & \\
\hline Contact for additional information & \\
\hline Additional accreditation/recognition & \\
\hline
\end{tabular}

\begin{tabular}{|l|l|}
\hline \multicolumn{2}{|c|}{ Petroleum Quality Testing } \\
\hline \hline Address & Telephone Number \\
\hline \hline NOT AVAILABLE & \\
\hline Person responsible for program & \\
\hline Contact for additional information & \\
\hline Additional accreditation/recognition & \\
\hline
\end{tabular}

\begin{tabular}{|l|l||}
\hline \multicolumn{2}{|c|}{ Additional Tests or Laboratory Functions } \\
\hline \hline Address & Telephone Number \\
\hline & \\
\hline Person responsible for program & \\
\hline Contact for additional information & \\
\hline Additional accreditation/recognition & \\
\hline
\end{tabular}




\section{Nebraska}

Accreditation Period: 1993

\begin{tabular}{|l|l|l|}
\hline Laboratory Staff & Address & Telephone Number \\
\hline \hline Gary Kliment, Metrologist & \multicolumn{1}{|c|}{$\begin{array}{l}\text { Division of Weights and Measures } \\
\text { PO Box 94757 } \\
\text { Lincoln, NE 68509 } \\
\text { NE Standards Lab, 3705 S 14th St } \\
\text { Lincoln, NE 68502 }\end{array}$} & $\begin{array}{l}\text { (402) 479-4171 Lab } \\
\text { FAX: (402) 471-0091 Lab }\end{array}$ \\
\hline
\end{tabular}

\begin{tabular}{|c|c|c|c|c|c|}
\hline \multicolumn{6}{|c|}{ Services Available } \\
\hline \multicolumn{3}{|c|}{ Tolerance Testing } & \multicolumn{3}{|c|}{ Calibration } \\
\hline \multirow[t]{2}{*}{ Mass } & Weight $\leq 1000 \mathrm{lb}$ & $\mathbf{F}$ & \multirow[t]{2}{*}{ Mass } & Weight $\leq 3 \mathrm{~kg}$ & $\mathrm{x}$ \\
\hline & Weight $>1000 \mathrm{lb}$ & $\mathrm{F}$ & & Weight $>3 \mathrm{~kg}$ & $\mathrm{x}$ \\
\hline \multirow[t]{3}{*}{ Volume } & Small $\leq 25$ gal & $\mathbf{F}$ & \multirow[t]{3}{*}{ Volume } & Metal Standards & $\mathrm{x}$ \\
\hline & Intermediate $25-500 \mathrm{gal}$ & $\mathbf{F}$ & & \multirow[t]{2}{*}{ Small Glassware } & \multirow[t]{2}{*}{$\mathrm{x}$} \\
\hline & Large > 500 gal & $\mathbf{F}$ & & & \\
\hline \multirow{4}{*}{$\begin{array}{l}\text { O: } \\
\mathrm{F}: \\
\mathrm{N}: \\
\mathrm{X}:\end{array}$} & \multirow{4}{*}{\multicolumn{2}{|c|}{$\begin{array}{l}\text { Service to parent organization only; } \\
\text { Service available on a fee basis; } \\
\text { Service available, no fee; } \\
\text { No accreditation, contact laboratory for availability. }\end{array}$}} & \multirow[t]{2}{*}{ Length } & Steel Tapes & $\mathrm{x}$ \\
\hline & & & & Rigid Rules & $\mathrm{x}$ \\
\hline & & & Frequency & Tuning Forks & $\mathrm{x}$ \\
\hline & & & Temperature & Liquid in Glass Thermometry & $\mathrm{x}$ \\
\hline
\end{tabular}

\section{Fees}

Labor...\$23 per hour. Plus materials, and time spent cleaning.

Mass: Class F: Other Sets:

Number of weights

\begin{tabular}{|c|c|c|}
\hline $1-14$ & $\$ 24$ & $\$ 30$ \\
\hline $15-25$ & 32 & 50 \\
\hline $26-38$ & 48 & 70 \\
\hline$>38$ & $\$ 1$ & additional weight \\
\hline
\end{tabular}

Length and Volume:

flasks: < gill: $\$ 6.50$; gill - pint: $\$ 9$; quart: $\$ 10.50$;

$1 / 2$ gal: $\$ 13.50$; gallon: $\$ 16.00$; $\leq 5$ gallon: $\$ 12 ; 5-25$ gallon: \$25; 25-100 gallon: \$35.

tapes: $3 \mathrm{ft}-100 \mathrm{ft}$ : $\$ 1$ per interval. 


\section{Nebraska}

\begin{tabular}{|l|l||}
\hline \multicolumn{2}{|c|}{ Grain Moisture Testing } \\
\hline \hline Address & Telephone Number \\
\hline $\begin{array}{l}\text { Nebraska Public Service Commission } \\
\text { PO Box } 94927 \\
\text { Lincoln, NE 68509-4927 }\end{array}$ & (402) 471-0235 \\
\hline Person responsible for program & John Fecht/Don Kendle \\
\hline Contact for additional information & \\
\hline Additional accreditation/recognition & \\
\hline
\end{tabular}

\begin{tabular}{||l|l||}
\hline \multicolumn{2}{|c|}{ Petroleum Quality Testing } \\
\hline \hline Address & Telephone Number \\
\hline NOT AVAILABLE & \\
\hline Person responsible for program & \\
\hline Contact for additional information & \\
\hline Additional accreditation/recognition & \\
\hline
\end{tabular}

\begin{tabular}{||l|l||}
\hline \multicolumn{2}{|c|}{ Additional Tests or Laboratory Functions } \\
\hline \hline Address & Telephone Number \\
\hline & \\
\hline Person responsible for program & \\
\hline Contact for additional information & \\
\hline Additional accreditation/recognition & \\
\hline
\end{tabular}




\section{Nevada}

Accreditation Period: 1993-1995

\begin{tabular}{|l|l||l||}
\hline \hline Laboratory Staff & Address & Telephone Number \\
\hline \hline Steve Grabski, Metrologist & & (702) 688-1166 \\
& NV Dept of Agriculture & FAX: (702) 688-1178 \\
& PO Box 11100 & \\
& Reno, NV 89510-1100 & \\
& Shipping: 2150 Frazer Ave \\
& Sparks, NV 89431 & \\
\hline
\end{tabular}

\begin{tabular}{|c|c|c|c|c|c|}
\hline \multicolumn{6}{|c|}{ Services Available } \\
\hline \multicolumn{3}{|c|}{ Tolerance Testing } & \multicolumn{3}{|c|}{ Calibration } \\
\hline \multirow[t]{2}{*}{ Mass } & Weight $\leq 1000 \mathrm{lb}$ & $\mathbf{F}$ & \multirow[t]{2}{*}{ Mass } & Weight $\leq 3 \mathrm{~kg}$ & $\mathbf{F}$ \\
\hline & Weight $>1000 \mathrm{lb}$ & $\boldsymbol{F}$ & & Weight $>3 \mathrm{~kg}$ & $\mathbf{F}$ \\
\hline \multirow[t]{3}{*}{ Volume } & Small $\leq 25$ gal & $\mathbf{F}$ & \multirow[t]{3}{*}{ Volume } & Metal Standards & $\mathbf{F}$ \\
\hline & Intermediate $25-500$ gal & $F$ & & \multirow[t]{2}{*}{ Small Glassware } & \multirow[t]{2}{*}{$\mathbf{F}$} \\
\hline & Large $>500$ gal & $\mathbf{F}$ & & & \\
\hline \multirow{4}{*}{$\begin{array}{l}\text { O: } \\
\text { F: } \\
\text { N: } \\
\text { X: }\end{array}$} & \multirow{4}{*}{\multicolumn{2}{|c|}{$\begin{array}{l}\text { Service to parent organization only; } \\
\text { Service available on a fee basis; } \\
\text { Service available, no fee; } \\
\text { No accreditation, contact laboratory for availability. }\end{array}$}} & \multirow[t]{2}{*}{ Length } & Steel Tapes & $\mathbf{F}$ \\
\hline & & & & Rigid Rules & $\mathbf{F}$ \\
\hline & & & Frequency & Tuning Forks & $\mathrm{X}$ \\
\hline & & & Temperature & Liquid in Glass Thermometry & $\mathrm{X}$ \\
\hline
\end{tabular}

\section{Fees}

Labor...\$20 per hour, plus parts and shipping costs. 


\section{Nevada}

\begin{tabular}{||l|l||}
\hline \multicolumn{2}{|c||}{ Grain Moisture Testing } \\
\hline \hline Address & Telephone Number \\
\hline \hline $\begin{array}{l}\text { NOT AVAILABLE } \\
\text { Historical Information: Was available years ago and } \\
\text { performed by the Dept of Agriculture Seed Analyst. } \\
\text { PO Box 11100 } \\
\text { Reno, NV 89510-1100 }\end{array}$ & $(702) 688-1180$ \\
\hline Person to contact for additional information & \\
\hline Contact for additional information & Randy Bradley, Seed Analyst \\
\hline Additional accreditation/recognition & \\
\hline
\end{tabular}

\begin{tabular}{||l|l||}
\hline \multicolumn{2}{|c||}{ Petroleum Quality Testing } \\
\hline \hline Address & Telephone Number \\
\hline \hline $\begin{array}{l}\text { PO Box 11100, 350 Capitol Hill St } \\
\text { Reno, NV 89510-1100 }\end{array}$ & $(702) 688-1180$ \\
\hline Person responsible for program & \\
\hline Contact for additional information & Robert Gronowski, Plant Industry Director \\
\hline Additional accreditation/recognition & Debbie Furrie, Petroleum Chemist \\
\hline
\end{tabular}

\begin{tabular}{||l|l||}
\hline \multicolumn{2}{|c||}{ Additional Tests or Laboratory Functions } \\
\hline \hline Address & Telephone Number \\
\hline \hline & \\
\hline Person responsible for program & \\
\hline Contact for additional information & \\
\hline Additional accreditation/recognition & \\
\hline
\end{tabular}




\section{New Hampshire}

Not Currently Accredited

\begin{tabular}{||l|l|l||}
\hline Laboratory Staff & Address & Telephone Number \\
\hline \hline Michael F. Grenier, Director/Metrologist & $\begin{array}{l}\text { NH Department of Agriculture } \\
\text { Caller Box 2042 } \\
\text { 10 Ferry St } \\
\text { Concord, NH 03301-2042 }\end{array}$ & FAX: (603) 271-1109 \\
\hline
\end{tabular}

\begin{tabular}{|c|c|c|c|c|c|}
\hline \multicolumn{6}{|c|}{ Services Available } \\
\hline \multicolumn{3}{|c|}{ Tolerance Testing } & \multicolumn{3}{|c|}{ Calibration } \\
\hline \multirow[t]{2}{*}{ Mass } & Weight $\leq 1000 \mathrm{lb}$ & $\mathrm{x}$ & \multirow[t]{2}{*}{ Mass } & Weight $\leq 3 \mathrm{~kg}$ & $\mathrm{x}$ \\
\hline & Weight $>1000 \mathrm{lb}$ & $\mathrm{x}$ & & Weight $>3 \mathrm{~kg}$ & $\mathrm{X}$ \\
\hline \multirow[t]{3}{*}{ Volume } & Small $\leq 25$ gal & $\mathrm{x}$ & \multirow[t]{3}{*}{ Volume } & Metal Standards & $\mathrm{x}$ \\
\hline & Intermediate 25 - 500 gal & $\mathrm{X}$ & & \multirow[t]{2}{*}{ Small Glassware } & \multirow[t]{2}{*}{$\mathrm{x}$} \\
\hline & Large $>500 \mathrm{gal}$ & $\mathrm{x}$ & & & \\
\hline \multirow{4}{*}{$\begin{array}{l}\text { O: } \\
\text { F: } \\
\text { N: } \\
\text { X: }\end{array}$} & \multirow{4}{*}{\multicolumn{2}{|c|}{$\begin{array}{l}\text { Service to parent organization only; } \\
\text { Service available on a fee basis; } \\
\text { Service available, no fee; } \\
\text { No accreditation, contact laboratory for availability. }\end{array}$}} & \multirow[t]{2}{*}{ Length } & Steel Tapes & $\mathrm{x}$ \\
\hline & & & & Rigid Rules & $\mathrm{X}$ \\
\hline & & & Frequency & Tuning Forks & $\mathrm{x}$ \\
\hline & & & Temperature & Liquid in Glass Thermometry & $\mathrm{x}$ \\
\hline
\end{tabular}

\section{Fees}

Labor...\$40 per hour.

Glass flasks: $\$ 15 ; \mathrm{V} \leq 5$-gal: $\$ 20 ; 5$ gal $<\mathrm{V} \leq 25$ gal: $\$ 20 ; 25$ gal $<\mathrm{V} \leq 150$ gal: $\$ 125$;

Compartment calibrations: 1st 100 gal: $\$ 40$; Each âddl 100 gal $\$ 1.50$

Class F Test weights, meeting specifications and tolerances contained in NIST Handbook 105-1:

30 lb weight kit: $\$ 45 ; 5,10,25,50$ lb weights: $\$ 10$ each

$\mathrm{W}<500 \mathrm{lb}$ : $\$ 10$ each; $\mathrm{W}>500 \mathrm{lb}: \$ 25$ each;

Length: Rigid rules: $\$ 10$; Steel tapes $\leq 50$ feet: $\$ 15 ; 50 \mathrm{ft}<\mathrm{L} \leq 100 \mathrm{ft}$ : $\$ 25$ each 


\section{New Hampshire}

\begin{tabular}{|l|l||}
\hline \multicolumn{2}{|c|}{ Grain Moisture Testing } \\
\hline \hline Address & Telephone Number \\
\hline \hline NOT AVAILABLE & \\
\hline Person responsible for program & \\
\hline Contact for additional information & \\
\hline Additional accreditation/recognition & \\
\hline
\end{tabular}

\begin{tabular}{|l|l||}
\hline \multicolumn{2}{|c|}{ Petroleum Quality Testing } \\
\hline \hline Address & Telephone Number \\
\hline \hline NOT AVAILABLE & \\
\hline Person responsible for program & \\
\hline Contact for additional information & \\
\hline Additional accreditation/recognition & \\
\hline \hline
\end{tabular}

\begin{tabular}{||l|l||}
\hline \multicolumn{2}{|c|}{ Additional Tests or Laboratory Functions } \\
\hline \hline Address & Telephone Number \\
\hline $\begin{array}{l}10 \text { Ferry St } \\
\text { Concord, NH } 03301-2042\end{array}$ & $(603) 271-3709$ \\
\hline Person responsible for program & Michael F. Grenier \\
\hline Contact for additional information & \\
\hline Additional accreditation/recognition & Testing of Wheel Load Weighers \\
\hline
\end{tabular}




\section{New Jersey}

Accreditation Period: 1992-1994

\begin{tabular}{||l|l|l||}
\hline Laboratory Staff & Address & Telephone Number \\
\hline \hline Pasquale D'Errico, Inspector I & & (908) 815-4840 \\
Walter L. Smith, Inspector II & $\begin{array}{l}\text { Office of Weights and Measures } \\
\text { 1261 US Rte 1 and 9 South } \\
\text { Avenel, NJ 07001 }\end{array}$ & \\
& & \\
\hline
\end{tabular}

\begin{tabular}{|c|c|c|c|c|c|}
\hline \multicolumn{6}{|c|}{ Services Available } \\
\hline \multicolumn{3}{|c|}{ Tolerance Testing } & \multicolumn{3}{|c|}{ Calibration } \\
\hline \multirow[t]{2}{*}{ Mass } & Weight $\leq 1000 \mathrm{lb}$ & $\mathrm{N}$ & \multirow[t]{2}{*}{ Mass } & Weight $\leq 3 \mathrm{~kg}$ & $\mathrm{~N}$ \\
\hline & Weight $>1000 \mathrm{lb}$ & $\mathrm{x}$ & & Weight $>3 \mathrm{~kg}$ & $\mathrm{~N}$ \\
\hline \multirow[t]{3}{*}{ Volume } & Small $\leq 25$ gal & $\mathrm{N}$ & \multirow[t]{3}{*}{ Volume } & Metal Standards & $\mathrm{N}$ \\
\hline & Intermediate $25-500 \mathrm{gal}$ & $\mathrm{N}$ & & \multirow[t]{2}{*}{ Small Glassware } & \multirow[t]{2}{*}{$N$} \\
\hline & Large $>500 \mathrm{gal}$ & $\mathrm{N}$ & & & \\
\hline \multirow{4}{*}{$\begin{array}{l}\text { O: } \\
\text { F: } \\
\text { N: } \\
X:\end{array}$} & \multirow{4}{*}{\multicolumn{2}{|c|}{$\begin{array}{l}\text { Service to parent organization only; } \\
\text { Service available on a fee basis; } \\
\text { Service available, no fee; } \\
\text { No accreditation, contact laboratory for availability. }\end{array}$}} & \multirow[t]{2}{*}{ Length } & Steel Tapes & $\mathrm{N}$ \\
\hline & & & & Rigid Rules & $\mathrm{N}$ \\
\hline & & & Frequency & Tuning Forks & $\mathrm{N}$ \\
\hline & & & Temperature & Liquid in Glass Thermometry & $\mathrm{x}$ \\
\hline
\end{tabular}

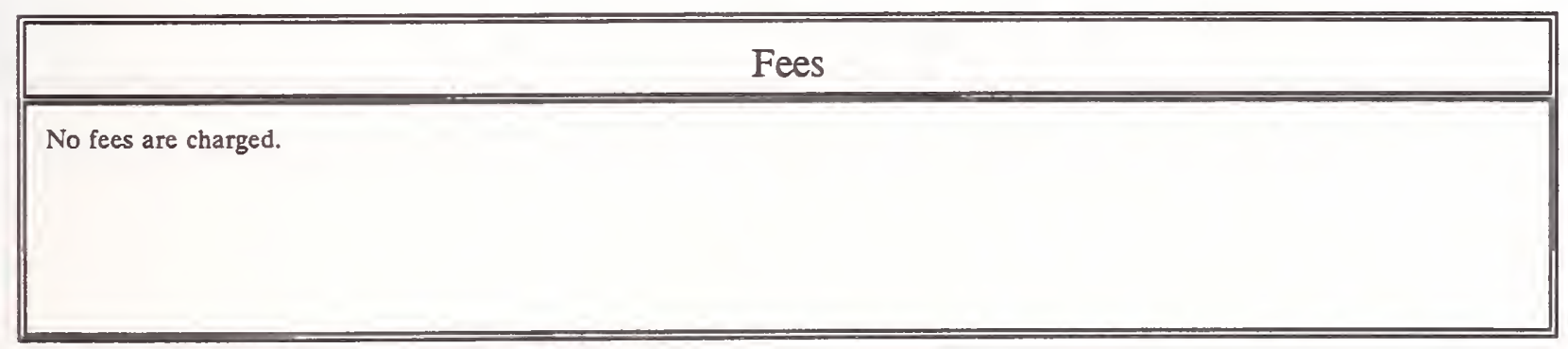

\section{Remarks:}

New Jersey moved into a new laboratory in 1988. Laboratory tests stopwatches, and also provides linear measurements using a special comparator table. 


\section{New Jersey}

\begin{tabular}{|l|l||}
\hline \multicolumn{2}{|c|}{ Grain Moisture Testing } \\
\hline \hline Address & Telephone Number \\
\hline & \\
& \\
\hline Person responsible for program & \\
\hline Contact for additional information & \\
\hline Additional accreditation/recognition & \\
\hline
\end{tabular}

\begin{tabular}{||l|l||}
\hline \multicolumn{2}{|c|}{ Petroleum Quality Testing } \\
\hline \hline Address & Telephone Number \\
\hline & \\
\hline Person responsible for program & \\
\hline Contact for additional information & \\
\hline Additional accreditation/recognition & \\
\hline
\end{tabular}

\begin{tabular}{||l|l||}
\hline \multicolumn{2}{|c|}{ Additional Tests or Laboratory Functions } \\
\hline \hline Address & Telephone Number \\
\hline & \\
\hline Person responsible for program & \\
\hline Contact for additional information & \\
\hline Additional accreditation/recognition & \\
\hline
\end{tabular}




\section{New Mexico}

Accreditation Period: 1991-1993

\begin{tabular}{|l|l|l|}
\hline Laboratory Staff & Address & Telephone Number \\
\hline Richard F. Schulmeister, Metrologist & $\begin{array}{l}\text { NM Dept of Agriculture } \\
\text { Standards and Consumer Services } \\
\text { Po Box 30005 } \\
\text { Dept 3170 } \\
\text { Las Cruces, NM 88003-0005 }\end{array}$ & FAX: (505) 646-3303 \\
\hline
\end{tabular}

\begin{tabular}{|c|c|c|c|c|c|}
\hline \multicolumn{6}{|c|}{ Services Available } \\
\hline \multicolumn{3}{|c|}{ Tolerance Testing } & \multicolumn{3}{|c|}{ Calibration } \\
\hline \multirow[t]{2}{*}{ Mass } & Weight $\leq 1000 \mathrm{lb}$ & F & \multirow[t]{2}{*}{ Mass } & Weight $\leq 3 \mathrm{~kg}$ & $\mathrm{~F}$ \\
\hline & Weight $>1000 \mathrm{lb}$ & $F$ & & Weight $>3 \mathrm{~kg}$ & $\mathrm{~F}$ \\
\hline \multirow[t]{3}{*}{ Volume } & Small $\leq 25$ gal & F & \multirow[t]{3}{*}{ Volume } & Metal Standards & F \\
\hline & Intermediate 25 - 500 ga & $\mathbf{F}$ & & \multirow[t]{2}{*}{ Small Glassware } & \multirow[t]{2}{*}{$\mathrm{F}$} \\
\hline & Large $>500 \mathrm{gal}$ & $\mathbf{F}$ & & & \\
\hline \multirow{4}{*}{$\begin{array}{l}\text { O: } \\
\text { F: } \\
\text { N: } \\
X:\end{array}$} & \multirow{4}{*}{\multicolumn{2}{|c|}{$\begin{array}{l}\text { Service to parent organization only, } \\
\text { Service available on a fee basis; } \\
\text { Service available, no fee; } \\
\text { No accreditation, contact laboratory for availability. }\end{array}$}} & \multirow[t]{2}{*}{ Length } & Steel Tapes & $\mathrm{F}$ \\
\hline & & & & Rigid Rules & F \\
\hline & & & Frequency & Tuning Forks & $\mathbf{F}$ \\
\hline & & & Temperature & Liquid in Glass Thermometry & $\mathrm{x}$ \\
\hline
\end{tabular}

\section{Fees}

Labor...\$28 per hour. Minimum 1/2 hour charge. 


\section{New Mexico}

\begin{tabular}{||l|l||}
\hline \multicolumn{2}{|c||}{ Grain Moisture Testing } \\
\hline \hline Address & Telephone Number \\
\hline \hline NOT AVAILABLE & \\
\hline Person responsible for program & \\
\hline Contact for additional information & \\
\hline Additional accreditation/recognition & \\
\hline \hline
\end{tabular}

\begin{tabular}{|l|l||}
\hline \multicolumn{2}{|c|}{ Petroleum Quality Testing } \\
\hline \hline Address & Telephone Number \\
\hline \hline $\begin{array}{l}\text { PO Box 30005, Dept 3170 } \\
\text { Corner of Gregg and Espina } \\
\text { Las Cruces, NM 88003-0005 }\end{array}$ & $\begin{array}{l}\text { (505) 646-1616 } \\
\text { FAX: (505) 646-3303 }\end{array}$ \\
\hline Person responsible for program & Gary West \\
\hline Contact for additional information & Floyd Highfill \\
\hline Additional accreditation/recognition & \\
\hline
\end{tabular}

\begin{tabular}{||l|l||}
\hline \multicolumn{2}{|c||}{ Additional Tests or Laboratory Functions } \\
\hline \hline Address & Telephone Number \\
\hline & \\
& \\
\hline Person responsible for program & \\
\hline Contact for additional information & \\
\hline Additional accreditation/recognition & \\
\hline
\end{tabular}




\section{New York}

Accreditation Period: 1993-1995

\begin{tabular}{|l||l|l||}
\hline Laboratory Staff & Address & Telephone Number \\
\hline \hline Ross J. Andersen, Asst. Director & & $\begin{array}{l}(518) 457-3452 \\
(518) 457-3146\end{array}$ \\
William Fishman, Metrologist & $\begin{array}{l}\text { Bureau of Weights and Measures } \\
\text { Bldg 7A State Campus } \\
\text { Albany, NY 12235 }\end{array}$ & FAX: (518) 457-4780 \\
Ed Szesnat, Metrologist & & \\
\hline
\end{tabular}

\begin{tabular}{|c|c|c|c|c|c|}
\hline \multicolumn{6}{|c|}{ Services Available } \\
\hline \multicolumn{3}{|c|}{ Tolerance Testing } & \multicolumn{3}{|c|}{ Calibration } \\
\hline \multirow[t]{2}{*}{ Mass } & Weight $\leq 1000 \mathrm{lb}$ & $\mathrm{F}$ & \multirow[t]{2}{*}{ Mass } & Weight $\leq 3 \mathrm{~kg}$ & $\mathrm{~F}$ \\
\hline & Weight $>1000 \mathrm{lb}$ up to $3000 \mathrm{lb}$ & $\mathrm{F}$ & & Weight $>3 \mathrm{~kg}$ & $\mathrm{~F}$ \\
\hline \multirow[t]{3}{*}{ Volume } & Small $\leq 25$ gal & $\mathbf{F}$ & \multirow[t]{3}{*}{ Volume } & Metal Standards & $\mathbf{F}$ \\
\hline & Intermediate 25 - $500 \mathrm{gal}$ & $\mathrm{F}$ & & \multirow[t]{2}{*}{ Small Glassware } & \multirow[t]{2}{*}{$\mathbf{F}$} \\
\hline & Large > $500 \mathrm{gal}$ & F & & & \\
\hline \multirow{4}{*}{$\begin{array}{l}\text { O: } \\
\text { F: } \\
\text { N: } \\
\text { X: }\end{array}$} & \multirow{4}{*}{\multicolumn{2}{|c|}{$\begin{array}{l}\text { Service to parent organization only; } \\
\text { Service available on a fee basis; } \\
\text { Service available, no fee; } \\
\text { No accreditation, contact laboratory for availability. }\end{array}$}} & \multirow[t]{2}{*}{ Length } & Steel Tapes & $\mathbf{F}$ \\
\hline & & & & Rigid Rules & $\mathbf{F}$ \\
\hline & & & Frequency & Tuning Forks & $\mathrm{x}$ \\
\hline & & & Temperature & Liquid in Glass Thermometry & $\mathbf{F}$ \\
\hline
\end{tabular}

\section{Fees}

Labor...Special Tests: $\$ 75$ per hour.

Fees vary according to type of artifact and type of service requested. Contact laboratory directly for specific fee information.

\section{Remarks:}

The New York laboratory also tests stopwatches and taximeters. 


\section{New York}

\begin{tabular}{||l|l||}
\hline \multicolumn{2}{|c||}{ Grain Moisture Testing } \\
\hline \hline Address & Telephone Number \\
\hline \hline NOT AVAILABLE & \\
\hline Person responsible for program & \\
\hline Contact for additional information & \\
\hline Additional accreditation/recognition & \\
\hline
\end{tabular}

\begin{tabular}{||l|l||}
\hline \multicolumn{2}{|c||}{ Petroleum Quality Testing } \\
\hline \hline Address & Telephone Number \\
\hline \hline $\begin{array}{l}\text { Bureau of Weights and Measures } \\
\text { I Winners Circle } \\
\text { Albany, NY } 12235\end{array}$ & $(518)$ 457-3146 \\
\hline Person responsible for program & John J. Bartfai \\
\hline Contact for additional information & Ross J. Andersen \\
\hline Additional accreditation/recognition & \\
\hline
\end{tabular}

\begin{tabular}{||l|l||}
\hline \multicolumn{2}{|c||}{ National Type Evaluation Program (NTEP) } \\
\hline \hline Address & Telephone Number \\
\hline \hline $\begin{array}{l}\text { Bureau of Weights and Measures } \\
\text { Bldg 7-A, State Campus } \\
\text { Albany, NY 12235 }\end{array}$ & $(518) 457-3452$ \\
\hline Person responsible for program & John J. Bartfai \\
\hline Contact for additional information & Ross J. Andersen, William Fishman, Ed Szesnat \\
\hline Additional accreditation/recognition & NIST Authorized Laboratory \\
\hline NTEP testing of scales, other weighing systems, and electronic cash registers interfaced with scales. \\
\hline
\end{tabular}




\section{North Carolina}

Accreditation Period: 1992-1994

\begin{tabular}{||l|l|l||}
\hline \hline Laboratory Staff & Address & Telephone Number \\
\hline \hline L. F. Eason, Standards Laboratory Manager & & NC Dept of Agriculture/Dept SD \\
Sherry Teachez, Metrologist II & $\begin{array}{l}\text { Standards Laboratory, PO Box 27647 } \\
\text { Raleigh, NC 27611 }\end{array}$ & FAX: (919) 733-3313 (office) \\
Sharon Denning, Metrologist I & $\begin{array}{l}\text { Shipping: 4040 District Drive, } \\
\text { Raleigh, NC 27607 }\end{array}$ & FAX: (919) 733-8804 (lab) \\
two vacant Metrologist I positions & & \\
Cheryl Tew, Grain Moisture Program Supervisor & & \\
\hline
\end{tabular}

\begin{tabular}{|c|c|c|c|c|c|}
\hline \multicolumn{6}{|c|}{ Services Available } \\
\hline \multicolumn{3}{|c|}{ Tolerance Testing } & \multicolumn{3}{|c|}{ Calibration } \\
\hline \multirow[t]{2}{*}{ Mass } & Weight $\leq 1000 \mathrm{lb}$ & $\mathbf{F}$ & \multirow[t]{2}{*}{ Mass } & Weight $\leq 3 \mathrm{~kg}$ & $\mathbf{F}$ \\
\hline & Weight > $1000 \mathrm{lb}$ & $\mathbf{F}$ & & Weight > $3 \mathrm{~kg}$ & $\mathbf{F}$ \\
\hline \multirow[t]{3}{*}{ Volume } & Small $\leq 25 \mathrm{gal}$ & *F & \multirow[t]{3}{*}{ Volume } & Metal Standards & *F \\
\hline & Intermediate 25 - $500 \mathrm{gal}$ & ${ }^{*} \mathrm{~F}$ & & \multirow[t]{2}{*}{ Small Glassware } & \multirow[t]{2}{*}{ F } \\
\hline & Large > $500 \mathrm{gal}$ & ${ }^{*} \mathrm{~F}$ & & & \\
\hline \multirow{4}{*}{$\begin{array}{l}\text { O: } \\
\text { F: } \\
\mathrm{N}: \\
\mathrm{X}:\end{array}$} & \multirow{4}{*}{\multicolumn{2}{|c|}{$\begin{array}{l}\text { Service to parent organization only; } \\
\text { Service available on a fee basis; } \\
\text { Service available, no fee; } \\
\text { No accreditation, contact laboratory for availability. }\end{array}$}} & \multirow[t]{2}{*}{ Length } & Steel Tapes & $\mathbf{F}$ \\
\hline & & & & Rigid Rules & F \\
\hline & & & Frequency & Tuning Forks & $\mathrm{X}$ \\
\hline & & & Temperature & Liquid in Glass Thermometry & $\mathrm{F}$ \\
\hline
\end{tabular}

\section{Fees}

- Fees are not charged for work related to petroleum measurements if devices are used within North Carolina.

This part of the program is funded by other fees. Fees are established pursuant to Section 1, Article 1 of Chapter $81 \mathrm{~A}$ of the General Statutes and vary according to type of artifact and type of service requested. Contact the laboratory directly for fee information.

\section{Remarks:}

North Carolina moved into a new laboratory in 1985 and has an additional large volume calibration facility in Wilmington, NC. 


\section{North Carolina}

\begin{tabular}{||l|l||}
\hline \multicolumn{2}{|c||}{ Grain Moisture Testing } \\
\hline \hline Address & Telephone Number \\
\hline \hline $\begin{array}{l}\text { NC Dept of Agriculture } \\
\text { Standards Laboratory } \\
\text { PO Box 27647 } \\
\text { Raleigh, NC } 27611\end{array}$ & $\begin{array}{l}(919) 733-4411 \\
\text { FAX: (919)-733-8804 }\end{array}$ \\
\hline Person responsible for program & Cheryl Tew \\
\hline Contact for additional information & L. F. Eason \\
\hline Additional accreditation/recognition & \\
\hline
\end{tabular}

\begin{tabular}{|l|l|}
\hline \multicolumn{2}{|c|}{ Petroleum Quality Testing } \\
\hline \hline Address & Telephone Number \\
\hline \hline $\begin{array}{l}\text { NC Dept of Agriculture } \\
\text { Motor Fuels Laboratory } \\
\text { PO Box } 27647 \\
\text { Raleigh, NC } 27611\end{array}$ & $\begin{array}{l}\text { (919) 733-3246 } \\
\text { FAX: (919) 733-2928 }\end{array}$ \\
\hline Person responsible for program & Duane Scott \\
\hline Contact for additional information & Winston Sutton \\
\hline Additional accreditation/recognition & National Regional Exchange Program, ASTM Member \\
\hline
\end{tabular}

\begin{tabular}{||l|l||}
\hline \multicolumn{2}{|c|}{ National Type Evaluation Program (NTEP) } \\
\hline \hline Address & Telephone Number \\
\hline \hline $\begin{array}{l}\text { NC Dept of Agriculture } \\
\text { Standards Division } \\
\text { PO Box } 27647 \\
\text { Raleigh, NC } 27611\end{array}$ & $\begin{array}{l}(919) 733-3313 \\
\text { FAX: (919) 715-0524 }\end{array}$ \\
\hline Person responsible for program & N. David Smith \\
\hline Contact for additional information & Ronald Murdock \\
\hline Additional accreditation/recognition & NIST Authorized Laboratory \\
\hline NTEP testing of field performance evaluations on liquid measuring devices. \\
\hline
\end{tabular}




\section{North Dakota}

Accreditation Period: 1993

\begin{tabular}{|l|l|l||}
\hline Laboratory Staff & Address & Telephone Number \\
\hline Alan Moch, Director & $\begin{array}{l}\text { Public Service Commission } \\
\text { Testing and Safety Division } \\
\text { State Capitol } \\
\text { Bismarck, ND 58505 }\end{array}$ & FAX: (701) 224-2410 224-2400 \\
\hline
\end{tabular}

\begin{tabular}{|c|c|c|c|c|c|}
\hline \multicolumn{6}{|c|}{ Services Available } \\
\hline \multicolumn{3}{|c|}{ Tolerance Testing } & \multicolumn{3}{|c|}{ Calibration } \\
\hline \multirow[t]{2}{*}{ Mass } & Weight $\leq 1000 \mathrm{lb}$ & F & \multirow[t]{2}{*}{ Mass } & Weight $\leq 3 \mathrm{~kg}$ & $\mathrm{~F}$ \\
\hline & Weight $>1000 \mathrm{lb}$ & $\mathbf{F}$ & & Weight $>3 \mathrm{~kg}$ & $\mathrm{x}$ \\
\hline \multirow[t]{3}{*}{ Volume } & Small $\leq 25$ gal & $\mathbf{F}$ & \multirow[t]{3}{*}{ Volume } & Metal Standards & F \\
\hline & Intermediate 25 - $500 \mathrm{gal}$ & $\mathbf{F}$ & & \multirow[t]{2}{*}{ Small Glassware } & \multirow[t]{2}{*}{$\mathrm{x}$} \\
\hline & Large > 500 gal & $\mathbf{F}$ & & & \\
\hline \multirow{4}{*}{$\begin{array}{l}\text { O: } \\
\mathrm{F}: \\
\mathrm{N}: \\
\mathrm{X}:\end{array}$} & \multirow{4}{*}{\multicolumn{2}{|c|}{$\begin{array}{l}\text { Service to parent organization only, } \\
\text { Service available on a fee basis; } \\
\text { Service available, no fee; } \\
\text { No accreditation, contact laboratory for availability. }\end{array}$}} & \multirow[t]{2}{*}{ Length } & Steel Tapes & $\mathbf{F}$ \\
\hline & & & & Rigid Rules & $\mathbf{F}$ \\
\hline & & & Frequency & Tuning Forks & $\mathrm{x}$ \\
\hline & & & Temperature & Liquid in Glass Thermometry & $\mathrm{x}$ \\
\hline
\end{tabular}

\section{Fees}

Labor...\$7.50 per quarter hour, per person. 


\section{North Dakota}

\begin{tabular}{||l|l||}
\hline \multicolumn{2}{|c||}{ Grain Moisture Testing } \\
\hline \hline Address & Telephone Number \\
\hline NOT AVAILABLE & \\
\hline Person responsible for program & \\
\hline Contact for additional information & \\
\hline Additional accreditation/recognition & \\
\hline
\end{tabular}

\begin{tabular}{|l|l||}
\hline \multicolumn{2}{|c||}{ Petroleum Quality Testing } \\
\hline \hline Address & Telephone Number \\
\hline $\begin{array}{l}\text { Health and Consolidated laboratories } \\
\text { PO Box 937 } \\
\text { Bismarck, ND 58502-0937 }\end{array}$ & (701) 221-6149 \\
\hline Person responsible for program & Peri Dura \\
\hline Contact for additional information & \\
\hline Additional accreditation/recognition & \\
\hline
\end{tabular}

\begin{tabular}{|l|l||}
\hline \multicolumn{2}{|c|}{ Additional Tests or Laboratory Functions } \\
\hline \hline Address & Telephone Number \\
\hline \hline & \\
\hline Person responsible for program & \\
\hline Contact for additional information & \\
\hline Additional accreditation/recognition & \\
\hline
\end{tabular}




\section{Ohio}

Accreditation Period: 1993

\begin{tabular}{|l|l|l||}
\hline \hline Laboratory Staff & Address & Telephone Number \\
\hline \hline Ken Johnson, Metrologist & $\begin{array}{l}\text { OH Dept of Agriculture } \\
\text { Division of Weights and Measures } \\
\text { 8995 E Main St/ Bldg 5 } \\
\text { Reynoldsburg, OH 43068 }\end{array}$ & FAX: (614) 866-4174 866-6361 \\
& & \\
\hline
\end{tabular}

\begin{tabular}{|c|c|c|c|c|c|}
\hline \multicolumn{6}{|c|}{ Services Available } \\
\hline \multicolumn{3}{|c|}{ Tolerance Testing } & \multicolumn{3}{|c|}{ Calibration } \\
\hline \multirow[t]{2}{*}{ Mass } & Weight $\leq 1000 \mathrm{lb}$ & $\mathbf{F}$ & \multirow[t]{2}{*}{ Mass } & Weight $\leq 3 \mathrm{~kg}$ & $\mathrm{~F}$ \\
\hline & Weight $>1000 \mathrm{lb}$ & $\mathbf{F}$ & & Weight $>3 \mathrm{~kg}$ & $\mathrm{~F}$ \\
\hline \multirow[t]{3}{*}{ Volume } & Small $\leq 25$ gal & $\mathbf{F}$ & \multirow[t]{3}{*}{ Volume } & Metal Standards & $\mathrm{F}$ \\
\hline & Intermediate $25-500 \mathrm{gal}$ & $\mathbf{F}$ & & \multirow[t]{2}{*}{ Small Glassware } & \multirow[t]{2}{*}{$\mathrm{F}$} \\
\hline & Large $>500 \mathrm{gal}$ & $\mathrm{x}$ & & & \\
\hline \multirow{4}{*}{$\begin{array}{l}\text { O: } \\
\text { F: } \\
\text { N: } \\
\text { X: }\end{array}$} & \multirow{4}{*}{\multicolumn{2}{|c|}{$\begin{array}{l}\text { Service to parent organization only, } \\
\text { Service available on a fee basis; } \\
\text { Service available, no fee; } \\
\text { No accreditation, contact laboratory for availability. }\end{array}$}} & \multirow[t]{2}{*}{ Length } & Steel Tapes & $\mathrm{F}$ \\
\hline & & & & Rigid Rules & $\mathrm{F}$ \\
\hline & & & Frequency & Tuning Forks & $\mathrm{x}$ \\
\hline & & & Temperature & Liquid in Glass Thermometry & $\mathrm{x}$ \\
\hline
\end{tabular}

\section{Fees}

Fees are established pursuant to Section 901.43 of the Ohio Revised Code and vary according to type of artifact and type of service requested. Contact the laboratory directly for fee information. 


\section{Ohio}

\begin{tabular}{|l|l||}
\hline \multicolumn{2}{|c|}{ Grain Moisture Testing } \\
\hline \hline Address & Telephone Number \\
\hline \hline NOT AVAILABLE & \\
\hline Person responsible for program & \\
\hline Contact for additional information & \\
\hline Additional accreditation/recognition & \\
\hline
\end{tabular}

\begin{tabular}{|l|l||}
\hline \multicolumn{2}{|c|}{ Petroleum Quality Testing } \\
\hline \hline Address & Telephone Number \\
\hline \hline NOT AVAILABLE & \\
& \\
\hline Person responsible for program & \\
\hline Contact for additional information & \\
\hline Additional accreditation/recognition & \\
\hline
\end{tabular}

\begin{tabular}{||l|l||}
\hline \multicolumn{2}{|c|}{ National Type Evaluation Program (NTEP) } \\
\hline \hline Address & Telephone Number \\
\hline $\begin{array}{l}\text { OH Department of Agriculture } \\
\text { Division of Weights and Measures } \\
8995 \text { E Main St/Bldg } 5 \\
\text { Reynoldsburg, OH } 43068\end{array}$ & $(614) 866-6361$ \\
\hline Person responsible for program & Jim Truex \\
\hline Contact for additional information & \\
\hline Additional accreditation/recognition & NIST Authorized Laboratory \\
\hline $\begin{array}{l}\text { NTEP testing of commercial scales, other weighing devices and electronic cash registers interfaced with scales. Performance } \\
\text { testing of retail motor fuel dispensers. }\end{array}$ \\
\hline
\end{tabular}




\section{Oklahoma}

Accreditation Period: 1993-1995

\begin{tabular}{||l|l||l||}
\hline Laboratory Staff & Address & Telephone Number \\
\hline Ken L. Fraley, Metrologist & $\begin{array}{l}\text { Bureau of Standards } \\
\text { Laboratory Division } \\
\text { 2800 N Lincoln Blvd } \\
\text { Oklahoma City, OK 73105 }\end{array}$ & FAX: (405) 521-4912 \\
& & \\
\hline
\end{tabular}

\begin{tabular}{|c|c|c|c|c|c|}
\hline \multicolumn{6}{|c|}{ Services Available } \\
\hline \multicolumn{3}{|c|}{ Tolerance Testing } & \multicolumn{3}{|c|}{ Calibration } \\
\hline \multirow[t]{2}{*}{ Mass } & Weight $\leq 1000 \mathrm{lb}$ & $\mathbf{F}$ & \multirow[t]{2}{*}{ Mass } & Weight $\leq 3 \mathrm{~kg}$ & $\mathbf{F}$ \\
\hline & Weight $>1000 \mathrm{lb}$ & $\mathbf{F}$ & & Weight $>3 \mathrm{~kg}$ & $\mathbf{F}$ \\
\hline \multirow[t]{3}{*}{ Volume } & Small $\leq 25$ gal & $\mathbf{F}$ & \multirow[t]{3}{*}{ Volume } & Metal Standards & $\mathbf{F}$ \\
\hline & Intermediate $25-500 \mathrm{gal}$ & $\mathbf{F}$ & & \multirow[t]{2}{*}{ Small Glassware } & \multirow[t]{2}{*}{$\mathrm{F}$} \\
\hline & Large > $500 \mathrm{gal}$ & $\mathrm{x}$ & & & \\
\hline \multirow{4}{*}{$\begin{array}{l}\text { O: } \\
\text { F: } \\
\text { N: } \\
\text { X: }\end{array}$} & \multirow{4}{*}{\multicolumn{2}{|c|}{$\begin{array}{l}\text { Service to parent organization only; } \\
\text { Service available on a fee basis; } \\
\text { Service available, no fee; } \\
\text { No accreditation, contact laboratory for availability. }\end{array}$}} & \multirow[t]{2}{*}{ Length } & Steel Tapes & $\mathbf{F}$ \\
\hline & & & & Rigid Rules & $\mathrm{F}$ \\
\hline & & & Frequency & Tuning Forks & $\mathrm{X}$ \\
\hline & & & Temperature & Liquid in Glass Thermometry & $\mathrm{X}$ \\
\hline
\end{tabular}

\section{Fees}

Labor...\$25 per hour for special tests not listed in the fee schedule.

Fees vary according to type of artifact and type of service requested. Contact laboratory directly for specific fee information.

\section{Remarks:}

Oklahoma moved into a new laboratory in 1986. The laboratory has sophisticated computer software for control charts and data analysis. 


\section{Oklahoma}

\begin{tabular}{|l|l|}
\hline \multicolumn{2}{|c|}{ Grain Moisture Testing } \\
\hline \hline Address & Telephone Number \\
\hline $\begin{array}{l}\text { Bureau of Standards } \\
\text { 2800 N Lincoln } \\
\text { Oklahoma City, OK } 73105\end{array}$ & (405) 521-3864 X370 \\
\hline Person responsible for program & Ken Fraley \\
\hline Contact for additional information & Richard Gonzales \\
\hline Additional accreditation/recognition & \\
\hline
\end{tabular}

\begin{tabular}{|l|l||}
\hline \multicolumn{2}{|c||}{ Petroleum Quality Testing } \\
\hline \hline Address & Telephone Number \\
\hline $\begin{array}{l}\text { Oklahoma Corporation Commission } \\
\text { Fuel Inspection Division } \\
130 \text { NE 23rd ST } \\
\text { Oklahoma City, OK 73105 }\end{array}$ & (405) 521-2487 \\
\hline Person responsible for program & Ron Hamilton \\
\hline Contact for additional information & \\
\hline Additional accreditation/recognition & \\
\hline
\end{tabular}

\begin{tabular}{||l|l||}
\hline \multicolumn{2}{|c|}{ Additional Tests or Laboratory Functions } \\
\hline \hline Address & Telephone Number \\
\hline & \\
\hline Person responsible for program & \\
\hline Contact for additional information & \\
\hline Additional accreditation/recognition & \\
\hline
\end{tabular}




\section{Oregon}

Accreditation Period: 1991-1993

\begin{tabular}{||l|l|l||}
\hline \hline Laboratory Staff & Address & Telephone Number \\
\hline \hline James F. Clifford, Metrologist & & (503) 378-5504 \\
& $\begin{array}{l}\text { OR Dept of Agriculture } \\
\text { Msmt Standards Division } \\
\text { 635 Capitol St NE } \\
\text { Salem, OR 97310-0110 }\end{array}$ & FAX: (503) 378-6525 \\
& & \\
\hline
\end{tabular}

\begin{tabular}{|c|c|c|c|c|c|}
\hline \multicolumn{6}{|c|}{ Services Available } \\
\hline \multicolumn{3}{|c|}{ Tolerance Testing } & \multicolumn{3}{|c|}{ Calibration } \\
\hline \multirow[t]{2}{*}{ Mass } & Weight $\leq 1000 \mathrm{lb}$ & $\mathrm{F}$ & \multirow[t]{2}{*}{ Mass } & Weight $\leq 3 \mathrm{~kg}$ & $\mathbf{F}$ \\
\hline & Weight > $1000 \mathrm{lb}$ & $\mathrm{F}$ & & Weight $>3 \mathrm{~kg}$ & $\mathbf{F}$ \\
\hline \multirow[t]{3}{*}{ Volume } & Small $\leq 25$ gal & F & \multirow[t]{3}{*}{ Volume } & Metal Standards & $\mathbf{F}$ \\
\hline & Intermediate $25-500 \mathrm{gal}$ & $\mathrm{F}$ & & \multirow[t]{2}{*}{ Small Glassware } & \multirow[t]{2}{*}{$\mathbf{F}$} \\
\hline & Large $>500$ gal & $\mathbf{F}$ & & & \\
\hline \multirow{4}{*}{$\begin{array}{l}\text { O: } \\
\text { F: } \\
\text { N: } \\
\text { X: }\end{array}$} & \multirow{4}{*}{\multicolumn{2}{|c|}{$\begin{array}{l}\text { Service to parent organization only; } \\
\text { Service available on a fee basis; } \\
\text { Service available, no fee; } \\
\text { No accreditation, contact laboratory for availability. }\end{array}$}} & \multirow[t]{2}{*}{ Length } & Steel Tapes & $\mathrm{F}$ \\
\hline & & & & Rigid Rules & $\mathrm{F}$ \\
\hline & & & Frequency & Tuning Forks & $\mathrm{X}$ \\
\hline & & & Temperature & Liquid in Glass Thermometry & $\mathrm{X}$ \\
\hline
\end{tabular}

\section{Fees}

Labor... $\$ 50$ per hour with $1 / 4 \mathrm{hr}$ minimum. 


\section{Oregon}

\begin{tabular}{|l|l||}
\hline \multicolumn{2}{|c|}{ Grain Moisture Testing } \\
\hline \hline Address & Telephone Number \\
\hline \hline NOT AVAILABLE & \\
\hline Person responsible for program & \\
\hline Contact for additional information & \\
\hline Additional accreditation/recognition & \\
\hline
\end{tabular}

\begin{tabular}{||l|l||}
\hline \multicolumn{2}{|c|}{ Petroleum Quality Testing } \\
\hline \hline Address & Telephone Number \\
\hline \hline $\begin{array}{l}\text { Measurement Standards Division } \\
\text { Oregon Department of Agriculture } \\
635 \text { Capitol St NE } \\
\text { Salem, OR 97310-0110 }\end{array}$ & $(503)$ 373-0964 \\
\hline Person responsible for program & \\
\hline Contact for additional information & Ken Simila \\
\hline Additional accreditation/recognition & \\
\hline
\end{tabular}

\begin{tabular}{||l|l||}
\hline \multicolumn{2}{|c|}{ National Type Evaluation Program (NTEP) } \\
\hline \hline Address & Telephone Number \\
\hline \hline $\begin{array}{l}\text { Oregon Department of Agriculture } \\
\text { Measurement Standards Division } \\
\text { 635 Capitol St NE } \\
\text { Salem, OR 97310-0110 }\end{array}$ & $(503) 378-3792$ \\
\hline Person responsible for program & George Shefcheck \\
\hline Contact for additional information & James F. Clifford \\
\hline Additional accreditation/recognition & NIST Authorized Laboratory \\
\hline NTEP field performance evaluations on vehicle, axle-load, livestock and floor scales. \\
\hline \hline
\end{tabular}




\section{Pennsylvania}

Accreditation Period: 1993-1995

\begin{tabular}{|c|c|c|}
\hline Laboratory Staff & Address & Telephone Number \\
\hline $\begin{array}{l}\text { John E. Fisher, Lab Division Chief } \\
\text { G. Edward Carpenter, Metrologist } \\
\text { Peter Millvan, Metrologist }\end{array}$ & $\begin{array}{l}\text { Dept of General Services } \\
2221 \text { Forster St } \\
\text { Room G-28 } \\
\text { Harrisburg, PA } 17125 \\
\text { Metrology Laboratory } \\
\text { B } 124 \text { Transportation and Safety Bldg } \\
\text { Commonwealth Ave \& North St } \\
\text { Harrisburg, PA } 17120\end{array}$ & $\begin{array}{l}\text { (717) } 787-6426 \\
\text { FAX: (717) 783-6241 }\end{array}$ \\
\hline
\end{tabular}

\begin{tabular}{|c|c|c|c|c|c|}
\hline \multicolumn{6}{|c|}{ Services Available } \\
\hline \multicolumn{3}{|c|}{ Tolerance Testing } & \multicolumn{3}{|c|}{ Calibration } \\
\hline \multirow[t]{2}{*}{ Mass } & Weight $\leq 1000 \mathrm{lb}$ & $\mathbf{N}$ & \multirow[t]{2}{*}{ Mass } & Weight $\leq 3 \mathrm{~kg}$ & $\mathrm{~N}$ \\
\hline & Weight $>1000 \mathrm{lb}$ & $\mathrm{N}$ & & Weight $>3 \mathrm{~kg}$ & $\mathrm{~N}$ \\
\hline \multirow[t]{3}{*}{ Volume } & Small $\leq 25$ gal & $\mathrm{N}$ & \multirow[t]{3}{*}{ Volume } & Metal Standards & $\mathrm{N}$ \\
\hline & Intermediate 25 - $500 \mathrm{gal}$ & $\mathrm{N}$ & & \multirow[t]{2}{*}{ Small Glassware } & \multirow[t]{2}{*}{$\mathrm{N}$} \\
\hline & Large $>500 \mathrm{gal}$ & $\mathrm{x}$ & & & \\
\hline \multirow{4}{*}{$\begin{array}{l}\text { O: } \\
\mathrm{F}: \\
\mathrm{N}: \\
\mathrm{X}:\end{array}$} & \multirow{4}{*}{\multicolumn{2}{|c|}{$\begin{array}{l}\text { Service to parent organization only, } \\
\text { Service available on a fee basis; } \\
\text { Service available, no fee; } \\
\text { No accreditation, contact laboratory for availability. }\end{array}$}} & \multirow[t]{2}{*}{ Length } & Steel Tapes & $\mathrm{N}$ \\
\hline & & & & Rigid Rules & $\mathrm{N}$ \\
\hline & & & Frequency & Tuning Forks & $\mathrm{X}$ \\
\hline & & & Temperature & Liquid in Glass Thermometry & $\mathrm{x}$ \\
\hline
\end{tabular}

\section{Fees}

No fees are charged. 


\section{Pennsylvania}

\begin{tabular}{||l|l||}
\hline \multicolumn{2}{|c||}{ Grain Moisture Testing } \\
\hline Address & Telephone Number \\
\hline \hline NOT AVAILABLE & \\
\hline Person responsible for program & \\
\hline Contact for additional information & \\
\hline Additional accreditation/recognition & \\
\hline
\end{tabular}

\begin{tabular}{||l|l||}
\hline \multicolumn{2}{|c||}{ Petroleum Quality Testing } \\
\hline \hline Address & Telephone Number \\
\hline \hline NOT AVALLABLE & \\
\hline Person responsible for program & \\
\hline Contact for additional information & \\
\hline Additional accreditation/recognition & \\
\hline \hline
\end{tabular}

\begin{tabular}{||l|l||}
\hline \multicolumn{2}{|c||}{ Additional Tests or Laboratory Functions } \\
\hline \hline Address & Telephone Number \\
\hline \hline NONE & \\
\hline Person responsible for program & \\
\hline Contact for additional information & \\
\hline Additional accreditation/recognition & \\
\hline \hline
\end{tabular}




\section{Puerto Rico}

Accreditation Period: 1992-1994

\begin{tabular}{||l|l|l||}
\hline \hline Laboratory Staff & Address & Telephone Number \\
\hline \hline Edmundo Rosario-Fred, Technician & $\begin{array}{l}\text { Commonwealth of Puerto Rico } \\
\text { Dept of Consumer Affairs } \\
\text { PO Box 41059 } \\
\text { Minillas Station } \\
\text { Santurce, Puerto Rico 00940 } \\
\end{array}$ & FAX: (809) 726-6570 \\
& Weights and Measures Laboratory \\
& T22 Hoare St \\
& Santurce, PR 00908 & \\
\hline \hline
\end{tabular}

\begin{tabular}{|c|c|c|c|c|c|}
\hline \multicolumn{6}{|c|}{ Services Available } \\
\hline \multicolumn{3}{|c|}{ Tolerance Testing } & \multicolumn{3}{|c|}{ Calibration } \\
\hline \multirow[t]{2}{*}{ Mass } & Weight $\leq 1000 \mathrm{lb}$ & $\mathrm{F}$ & \multirow[t]{2}{*}{ Mass } & Weight $\leq 3 \mathrm{~kg}$ & $\mathrm{~F}$ \\
\hline & Weight $>1000 \mathrm{lb}$ & F & & Weight $>3 \mathrm{~kg}$ & $\mathrm{~F}$ \\
\hline \multirow[t]{3}{*}{ Volume } & Small $\leq 25$ gal & $\mathbf{F}$ & \multirow[t]{3}{*}{ Volume } & Metal Standards & $\mathrm{F}$ \\
\hline & Intermediate $25-500 \mathrm{gal}$ & $\mathrm{F}$ & & \multirow[t]{2}{*}{ Small Glassware } & \multirow[t]{2}{*}{$\mathrm{F}$} \\
\hline & Large > 500 gal & $\mathbf{F}$ & & & \\
\hline \multirow{4}{*}{$\begin{array}{l}\text { O: } \\
\text { F: } \\
\text { N: } \\
X:\end{array}$} & \multirow{4}{*}{\multicolumn{2}{|c|}{$\begin{array}{l}\text { Service to parent organization only; } \\
\text { Service available on a fee basis; } \\
\text { Service available, no fee; } \\
\text { No accreditation, contact laboratory for availability. }\end{array}$}} & \multirow[t]{2}{*}{ Length } & Steel Tapes & $\mathrm{F}$ \\
\hline & & & & Rigid Rules & $\mathrm{F}$ \\
\hline & & & Frequency & Tuning Forks & $\mathrm{x}$ \\
\hline & & & Temperature & Liquid in Glass Thermometry & $\mathrm{x}$ \\
\hline
\end{tabular}

\section{Fees}

Fees are established in accordance with the Law of Weights and Measures of the Commonwealth of Puerto Rico jointly with the

Organic Law of the Department of Consumer Affairs. Fees vary according to type of artifact and type of service requested.

\section{Remarks:}

Laboratory also tests density hydrometers used to test sugars, refractometers, and polarimeters. 


\section{Puerto Rico}

\begin{tabular}{||l|l||}
\hline \multicolumn{2}{|c|}{ Grain Moisture Testing } \\
\hline \hline Address & Telephone Number \\
\hline NOT AVAILABLE & \\
\hline Person responsible for program & \\
\hline Contact for additional information & \\
\hline Additional accreditation/recognition & \\
\hline
\end{tabular}

\begin{tabular}{||l|l|}
\hline \multicolumn{2}{|c|}{ Petroleum Quality Testing } \\
\hline \hline Address & Telephone Number \\
\hline \hline $\begin{array}{l}\text { Department of Consumer Affairs } \\
722 \text { Hoare St } \\
\text { Santurce, PR 00908 }\end{array}$ & $(809) 724-5153$ \\
\hline Person responsible for program & \\
\hline Contact for additional information & Heliodoro Lopez Torres \\
\hline Additional accreditation/recognition & Heliodoro Lopez Torres \\
\hline
\end{tabular}

\begin{tabular}{||l|l||}
\hline \multicolumn{2}{|c||}{ Additional Tests or Laboratory Functions } \\
\hline \hline Address & Telephone Number \\
\hline & \\
\hline Person responsible for program & \\
\hline Contact for additional information & \\
\hline Additional accreditation/recognition & \\
\hline
\end{tabular}




\section{Rhode Island}

Not Currently Accredited

\begin{tabular}{||l|l||l||}
\hline \hline Laboratory Staff & Address & Telephone Number \\
\hline \hline Lynda Agresti Maurer, Supervising Metrologist & & (401) 457-1867 \\
& $\begin{array}{l}\text { Dept of Labor } \\
\text { 220 Elmwood Ave } \\
\text { Providence, RI 02907 }\end{array}$ & \\
& & \\
\hline
\end{tabular}

\begin{tabular}{|c|c|c|c|c|c|}
\hline \multicolumn{6}{|c|}{ Services Available } \\
\hline \multicolumn{3}{|c|}{ Tolerance Testing } & \multicolumn{3}{|c|}{ Calibration } \\
\hline \multirow[t]{2}{*}{ Mass } & Weight $\leq 1000 \mathrm{lb}$ & $\mathrm{x}$ & \multirow[t]{2}{*}{ Mass } & Weight $\leq 3 \mathrm{~kg}$ & $\mathrm{x}$ \\
\hline & Weight $>1000 \mathrm{lb}$ & $\mathrm{x}$ & & Weight $>3 \mathrm{~kg}$ & $\mathrm{x}$ \\
\hline \multirow[t]{3}{*}{ Volume } & Small $\leq 25$ gal & $\mathrm{x}$ & \multirow[t]{3}{*}{ Volume } & Metal Standards & $\mathrm{x}$ \\
\hline & Intermediate $25-500 \mathrm{gal}$ & $\mathrm{x}$ & & \multirow[t]{2}{*}{ Small Glassware } & \multirow[t]{2}{*}{$\mathrm{x}$} \\
\hline & Large $>500 \mathrm{gal}$ & $\mathrm{x}$ & & & \\
\hline \multirow{4}{*}{$\begin{array}{l}\text { O: } \\
\text { F: } \\
\text { N: } \\
\text { X: }\end{array}$} & \multirow{4}{*}{\multicolumn{2}{|c|}{$\begin{array}{l}\text { Service to parent organization only; } \\
\text { Service available on a fee basis; } \\
\text { Service available, no fee; } \\
\text { No accreditation, contact laboratory for availability. }\end{array}$}} & \multirow[t]{2}{*}{ Length } & Steel Tapes & $\mathrm{x}$ \\
\hline & & & & Rigid Rules & $\mathrm{x}$ \\
\hline & & & Frequency & Tuning Forks & $\mathrm{x}$ \\
\hline & & & Temperature & Liquid in Glass Thermometry & $\mathrm{x}$ \\
\hline
\end{tabular}

\section{Fees}

No fees are charged.

\section{Remarks:}

Laboratory is not operated on a full-time basis. 


\section{Rhode Island}

\begin{tabular}{||l|l||}
\hline \multicolumn{2}{|c||}{ Grain Moisture Testing } \\
\hline \hline Address & Telephone Number \\
\hline \hline NOT AVAILABLE & \\
\hline Person responsible for program & \\
\hline Contact for additional information & \\
\hline Additional accreditation/recognition & \\
\hline
\end{tabular}

\begin{tabular}{||l|l||}
\hline \multicolumn{2}{|c|}{ Petroleum Quality Testing } \\
\hline \hline Address & Telephone Number \\
\hline \hline $\begin{array}{l}\text { Tax Administration } \\
\text { One Capitol Hill } \\
\text { Providence, RI 02908-5800 }\end{array}$ & $(401)$ 277-3050 \\
\hline Person responsible for program & \\
\hline Contact for additional information & Gary Clark \\
\hline Additional accreditation/recognition & Robert Geruso \\
\hline
\end{tabular}

\begin{tabular}{||l|l||}
\hline \multicolumn{2}{|c||}{ Additional Tests or Laboratory Functions } \\
\hline \hline Address & Telephone Number \\
\hline \hline & \\
\hline Person responsible for program & \\
\hline Contact for additional information & \\
\hline Additional accreditation/recognition & \\
\hline
\end{tabular}




\section{South Carolina}

Accreditation Period: 1993-1995

\begin{tabular}{|l|l|l||}
\hline Laboratory Staff & Address & Telephone Number \\
\hline \hline John V. Pugh, Metrology Laboratory Dir & $\begin{array}{l}\text { Dept of Agriculture/Cons Protection } \\
\text { Po Box 11280 } \\
\text { Columbia, SC 29211 } \\
\text { Shipping: 237 Catawba St } \\
\text { Columbia, SC 29201 }\end{array}$ & $\begin{array}{l}\text { (803) 737-2080 (office) } \\
\text { FAX: (803) } 253-4052 \text { (lab) }\end{array}$ \\
\hline
\end{tabular}

\begin{tabular}{|c|c|c|c|c|c|}
\hline \multicolumn{6}{|c|}{ Services Available } \\
\hline \multicolumn{3}{|c|}{ Tolerance Testing } & \multicolumn{3}{|c|}{ Calibration } \\
\hline \multirow[t]{2}{*}{ Mass } & Weight $\leq 1000 \mathrm{lb}$ & $\mathbf{N}$ & \multirow[t]{2}{*}{ Mass } & Weight $\leq 3 \mathrm{~kg}$ & $\mathrm{~N}$ \\
\hline & Weight $>1000 \mathrm{lb}$ & $\mathrm{N}$ & & Weight $>3 \mathrm{~kg}$ & $\mathrm{~N}$ \\
\hline \multirow[t]{3}{*}{ Volume } & Small $\leq 25$ gal & $\mathbf{N}$ & \multirow[t]{3}{*}{ Volume } & Metal Standards & $\mathrm{N}$ \\
\hline & Intermediate $25-500 \mathrm{gal}$ & $\mathrm{N}$ & & \multirow[t]{2}{*}{ Small Glassware } & \multirow[t]{2}{*}{$\mathrm{N}$} \\
\hline & Large $>500 \mathrm{gal}$ & $\mathrm{N}$ & & & \\
\hline \multirow{4}{*}{$\begin{array}{l}\text { O: } \\
\text { F: } \\
\text { N: } \\
\text { X: }\end{array}$} & \multirow{4}{*}{\multicolumn{2}{|c|}{$\begin{array}{l}\text { Service to parent organization only; } \\
\text { Service available on a fee basis; } \\
\text { Service available, no fee; } \\
\text { No accreditation, contact laboratory for availability. }\end{array}$}} & \multirow[t]{2}{*}{ Length } & Steel Tapes & $\mathrm{N}$ \\
\hline & & & & Rigid Rules & $\mathrm{N}$ \\
\hline & & & Frequency & Tuning Forks & $\mathrm{X}$ \\
\hline & & & Temperature & Liquid in Glass Thermometry & $\mathrm{X}$ \\
\hline
\end{tabular}

\section{Fees}

No fees are charged. 


\section{South Carolina}

\begin{tabular}{||l|l||}
\hline \multicolumn{2}{|c|}{ Grain Moisture Testing } \\
\hline \hline Address & Telephone Number \\
\hline \hline $\begin{array}{l}\text { PO Box } 11280 \\
\text { Columbia, SC } 29211\end{array}$ & $(803) 253-4052$ \\
& \\
\hline Person responsible for program & John V. Pugh \\
\hline Contact for additional information & \\
\hline Additional accreditation/recognition & \\
\hline
\end{tabular}

\begin{tabular}{||l|l||}
\hline \multicolumn{2}{|c|}{ Petroleum Quality Testing } \\
\hline \hline Address & Telephone Number \\
\hline $\begin{array}{l}\text { PO Box 11280 } \\
\text { Columbia, SC } 29211\end{array}$ & \\
& \\
\hline Person responsible for program & Dr. Thomas W. Brooks \\
\hline Contact for additional information & \\
\hline Additional accreditation/recognition & \\
\hline
\end{tabular}

\begin{tabular}{|l|l||}
\hline \multicolumn{2}{|c||}{ Additional Tests or Laboratory Functions } \\
\hline \hline Address & Telephone Number \\
\hline \hline & \\
\hline Person responsible for program & \\
\hline Contact for additional information & \\
\hline Additional accreditation/recognition & \\
\hline
\end{tabular}




\section{South Dakota}

Accreditation Period: 1993

\begin{tabular}{|l|l|l|}
\hline Laboratory Staff & Address & Telephone Number \\
\hline \hline James Peskey, Sr. Retail Inspector & $\begin{array}{l}\text { Division of Commercial Inspection } \\
\text { and Regulation } \\
\text { Office of Weights and Measures } \\
\text { 118 W Capitol } \\
\text { Pierre, SD 57501 }\end{array}$ & FAX: (605) 773-5825 \\
Paul Merriman, Inspector & & \\
\hline
\end{tabular}

\begin{tabular}{|c|c|c|c|c|c|}
\hline \multicolumn{6}{|c|}{ Services Available } \\
\hline \multicolumn{3}{|c|}{ Tolerance Testing } & \multicolumn{3}{|c|}{ Calibration } \\
\hline \multirow[t]{2}{*}{ Mass } & Weight $\leq 1000 \mathrm{lb}$ & F & \multirow[t]{2}{*}{ Mass } & Weight $\leq 3 \mathrm{~kg}$ & $\mathrm{X}$ \\
\hline & Weight $>1000 \mathrm{lb}$ & $\mathbf{F}$ & & Weight $>3 \mathrm{~kg}$ & $\mathrm{X}$ \\
\hline \multirow[t]{3}{*}{ Volume } & Small $\leq 25$ gal & $\mathbf{F}$ & \multirow[t]{3}{*}{ Volume } & Metal Standards & $\mathrm{X}$ \\
\hline & Intermediate $25-500 \mathrm{gal}$ & $\mathrm{X}$ & & \multirow[t]{2}{*}{ Small Glassware } & \multirow[t]{2}{*}{$\mathrm{X}$} \\
\hline & Large > 500 gal & $\mathrm{X}$ & & & \\
\hline \multirow{4}{*}{$\begin{array}{l}\text { O: } \\
\mathrm{F}: \\
\mathrm{N}: \\
\mathrm{X}:\end{array}$} & \multirow{4}{*}{\multicolumn{2}{|c|}{$\begin{array}{l}\text { Service to parent organization only; } \\
\text { Service available on a fee basis; } \\
\text { Service available, no fee; } \\
\text { No accreditation, contact laboratory for availability. }\end{array}$}} & \multirow[t]{2}{*}{ Length } & Steel Tapes & $\mathrm{X}$ \\
\hline & & & & Rigid Rules & $\mathrm{X}$ \\
\hline & & & Frequency & Tuning Forks & $\mathrm{X}$ \\
\hline & & & Temperature & Liquid in Glass Thermometry & $\mathrm{X}$ \\
\hline
\end{tabular}

\section{Fees}

Labor...\$20 per hour.

\section{Remarks:}

Laboratory is not operated on a full-time basis. 


\section{South Dakota}

\begin{tabular}{||l|l||}
\hline \multicolumn{2}{|c|}{ Grain Moisture Testing } \\
\hline \hline Address & Telephone Number \\
\hline \hline NOT AVAILABLE & \\
\hline Person responsible for program & \\
\hline Contact for additional information & \\
\hline Additional accreditation/recognition & \\
\hline
\end{tabular}

\begin{tabular}{||l|l||}
\hline \multicolumn{2}{|c|}{ Petroleum Quality Testing } \\
\hline \hline Address & Telephone Number \\
\hline \hline See laboratory address & \\
\hline Person responsible for program & \\
\hline Contact for additional information & James Peskey, Sr. Retail Inspector \\
\hline Additional accreditation/recognition & Michael Kumm, Director \\
\hline
\end{tabular}

\begin{tabular}{|l|l||}
\hline \multicolumn{2}{|c||}{ Additional Tests or Laboratory Functions } \\
\hline \hline Address & Telephone Number \\
\hline \hline & \\
\hline Person responsible for program & \\
\hline Contact for additional information & \\
\hline Additional accreditation/recognition & \\
\hline \hline
\end{tabular}




\section{Tennessee}

Not Currently Accredited

\begin{tabular}{|c|c|c|}
\hline Laboratory Staff & Address & Telephone Number \\
\hline Robert G. Williams, Supervisor & $\begin{array}{l}\text { TN Dept of Agriculture } \\
\text { PO Box } 40627 \text { Melrose Station } \\
\text { Nashville, TN } 37204\end{array}$ & $\begin{array}{l}\text { (615) 360-0159 Office } \\
\text { (615) 360-0158 Lab } \\
\text { FAX: (615) 360-0194 }\end{array}$ \\
\hline
\end{tabular}

\begin{tabular}{|c|c|c|c|c|c|}
\hline \multicolumn{6}{|c|}{ Services Available } \\
\hline \multicolumn{3}{|c|}{ Tolerance Testing } & \multicolumn{3}{|c|}{ Calibration } \\
\hline \multirow[t]{2}{*}{ Mass } & Weight $\leq 1000 \mathrm{lb}$ & $\mathrm{x}$ & \multirow[t]{2}{*}{ Mass } & Weight $\leq 3 \mathrm{~kg}$ & $\mathrm{x}$ \\
\hline & Weight $>1000 \mathrm{lb}$ & $\mathrm{x}$ & & Weight $>3 \mathrm{~kg}$ & $\mathrm{x}$ \\
\hline \multirow[t]{3}{*}{ Volume } & Small $\leq 25$ gal & $\mathrm{x}$ & \multirow[t]{3}{*}{ Volume } & Metal Standards & $\mathrm{x}$ \\
\hline & Intermediate $25-500 \mathrm{gal}$ & $\mathrm{x}$ & & \multirow[t]{2}{*}{ Small Glassware } & \multirow[t]{2}{*}{$\mathrm{x}$} \\
\hline & Large $>500 \mathrm{gal}$ & $\mathrm{x}$ & & & \\
\hline \multirow{4}{*}{$\begin{array}{l}\text { O: } \\
\text { F: } \\
\text { N: } \\
X:\end{array}$} & \multirow{4}{*}{\multicolumn{2}{|c|}{$\begin{array}{l}\text { Service to parent organization only; } \\
\text { Service available on a fee basis; } \\
\text { Service available, no fee; } \\
\text { No accreditation, contact laboratory for availability. }\end{array}$}} & \multirow[t]{2}{*}{ Length } & Steel Tapes & $\mathrm{x}$ \\
\hline & & & & Rigid Rules & $\mathrm{x}$ \\
\hline & & & Frequency & Tuning Forks & $\mathrm{x}$ \\
\hline & & & Temperature & Liquid in Glass Thermometry & $\mathrm{x}$ \\
\hline
\end{tabular}

\section{Fees}

No fees are charged. 


\section{Tennessee}

\begin{tabular}{||l|l||}
\hline \multicolumn{2}{|c||}{ Grain Moisture Testing } \\
\hline \hline Address & Telephone Number \\
\hline $\begin{array}{l}\text { TN Dept of Agriculture } \\
\text { Quality and Standards } \\
\text { PO Box 40627 } \\
\text { Nashville, TN } 37204\end{array}$ & (615) 360-0159 \\
\hline Person responsible for program & \\
\hline Contact for additional information & Robert G. Williams, Standards Administrator \\
\hline Additional accreditation/recognition & Cathryn Pittman, Technologist \\
\hline
\end{tabular}

\begin{tabular}{||l|l||}
\hline \multicolumn{2}{|c||}{ Petroleum Quality Testing } \\
\hline \hline Address & Telephone Number \\
\hline \hline $\begin{array}{l}\text { TN Dept of Agriculture } \\
\text { Quality and Standards } \\
\text { Holeman Bldg, PO Box } 40627 \\
\text { Nashville, TN } 37204\end{array}$ & $(615) 360-0147$ \\
\hline Person responsible for program & \\
\hline Contact for additional information & Randy Jennings, Petroleum Administrator \\
\hline Additional accreditation/recognition & \\
\hline
\end{tabular}

\begin{tabular}{|l|l||}
\hline \multicolumn{2}{|c|}{ Additional Tests or Laboratory Functions } \\
\hline \hline Address & Telephone Number \\
\hline \hline & \\
\hline Person responsible for program & \\
\hline Contact for additional information & \\
\hline Additional accreditation/recognition & \\
\hline
\end{tabular}




\section{Texas}

Accreditation Period: 1993-1995

\begin{tabular}{||l|l|l||}
\hline Laboratory Staff & Address & Telephone Number \\
\hline $\begin{array}{l}\text { James H. Eskew, Administrator of Metrology } \\
\text { Program }\end{array}$ & $\begin{array}{l}\text { TX Dept of Agriculture } \\
\text { Metrology Laboratory } \\
\text { 119 Cumberland Rd } \\
\text { Austin, TX 78704 }\end{array}$ & FAX: (512) 475-1642 \\
Rodger Brown, Metrologist & & $\begin{array}{l}\text { Lubbock Laboratory: } \\
\text { (806) 799-8555 }\end{array}$ \\
Randy Calcote, Metrologist, Lubbock & & \\
& & \\
\hline
\end{tabular}

\begin{tabular}{|c|c|c|c|c|c|}
\hline \multicolumn{6}{|c|}{ Services Available } \\
\hline \multicolumn{3}{|c|}{ Tolerance Testing } & \multicolumn{3}{|c|}{ Calibration } \\
\hline \multirow[t]{2}{*}{ Mass } & Weight $\leq 1000 \mathrm{lb}$ & $\mathbf{F}$ & \multirow[t]{2}{*}{ Mass } & Weight $\leq 3 \mathrm{~kg}$ & F \\
\hline & Weight $>1000 \mathrm{lb}$ & $\mathbf{F}$ & & Weight $>3 \mathrm{~kg}$ & $\mathbf{F}$ \\
\hline \multirow[t]{3}{*}{ Volume } & Small $\leq 25$ gal & $\mathbf{F}$ & \multirow[t]{3}{*}{ Volume } & Metal Standards & $\mathbf{F}$ \\
\hline & Intermediate $25-500 \mathrm{gal}$ & $\mathbf{F}$ & & \multirow[t]{2}{*}{ Small Glassware } & \multirow[t]{2}{*}{ F } \\
\hline & Large $>500 \mathrm{gal}$ & $\mathbf{F}$ & & & \\
\hline \multirow{4}{*}{$\begin{array}{l}\text { O: } \\
\text { F: } \\
\text { N: } \\
X:\end{array}$} & \multirow{4}{*}{\multicolumn{2}{|c|}{$\begin{array}{l}\text { Service to parent organization only; } \\
\text { Service available on a fee basis; } \\
\text { Service available, no fee; } \\
\text { No accreditation, contact laboratory for availability. }\end{array}$}} & \multirow[t]{2}{*}{ Length } & Steel Tapes & $\mathbf{F}$ \\
\hline & & & & Rigid Rules & $\mathbf{F}$ \\
\hline & & & Frequency & Tuning Forks & $\mathbf{x}$ \\
\hline & & & Temperature & Liquid in Glass Thermometry & $\mathrm{x}$ \\
\hline
\end{tabular}

\section{Fees}

Mass, Tolerance Testing: $W<10 \mathrm{lb}: \$ 2 ; 10 \leq \mathrm{W}<500 \mathrm{lb}: \$ 5 ; 500 \leq \mathrm{W}<2500 \mathrm{lb}: \$ 10 ; \mathrm{W} \geq 2500 \mathrm{lb}: \$ 20$.

Mass, Calibrations: $W \leq 3 \mathrm{~kg}$. $\$ 25$ each; $3 \mathrm{~kg}<W \leq 30 \mathrm{~kg}$ : $\$ 50$ each; $W>30 \mathrm{~kg}$ : $\$ 70$ each.

Volume: $\mathrm{V} \leq 5$ gal: $\$ 10 ; \mathrm{V}>5$ gal: $\$ 10$ plus $\$ 0.20$ per gal over 5 gallons; glassware: $\$ 25$ per increment.

Length: \$25 per increment.

\section{Remarks:}

Texas has independent laboratory rooms, balances, and standards for calibration and tolerance testing of mass. Calibrations up to $2500 \mathrm{lb}$, and tolerance testing up to $5000 \mathrm{lb}$ are possible. 


\section{Texas}

\begin{tabular}{|l|l||}
\hline \multicolumn{2}{|c|}{ Grain Moisture Testing } \\
\hline \hline Address & Telephone Number \\
\hline \hline NOT AVAILABLE & \\
\hline Person responsible for program & \\
\hline Contact for additional information & \\
\hline Additional accreditation/recognition & \\
\hline
\end{tabular}

\begin{tabular}{|l|l||}
\hline \multicolumn{2}{|c||}{ Petroleum Quality Testing } \\
\hline \hline Address & Telephone Number \\
\hline \hline NOT AVAILABLE & \\
& \\
\hline Person responsible for program & \\
\hline Contact for additional information & \\
\hline Additional accreditation/recognition & \\
\hline
\end{tabular}

\begin{tabular}{||l|l||}
\hline \multicolumn{2}{|c||}{ Additional Tests or Laboratory Functions } \\
\hline \hline Address & Telephone Number \\
\hline & \\
\hline Person responsible for program & \\
\hline Contact for additional information & \\
\hline Additional accreditation/recognition & \\
\hline
\end{tabular}




\section{Utah}

Accreditation Period: 1993-1994

\begin{tabular}{|l|l|l||}
\hline Laboratory Staff & Address & Telephone Number \\
\hline \hline Anthony De Arcose, Metrologist & $\begin{array}{l}\text { UT Dept of Agriculture } \\
\text { Weights and Measures Division } \\
\text { 350 N Redwood Rd } \\
\text { Salt Lake City, UT 84116 }\end{array}$ & FAX: (801) 538-7126 538-7153 \\
\hline
\end{tabular}

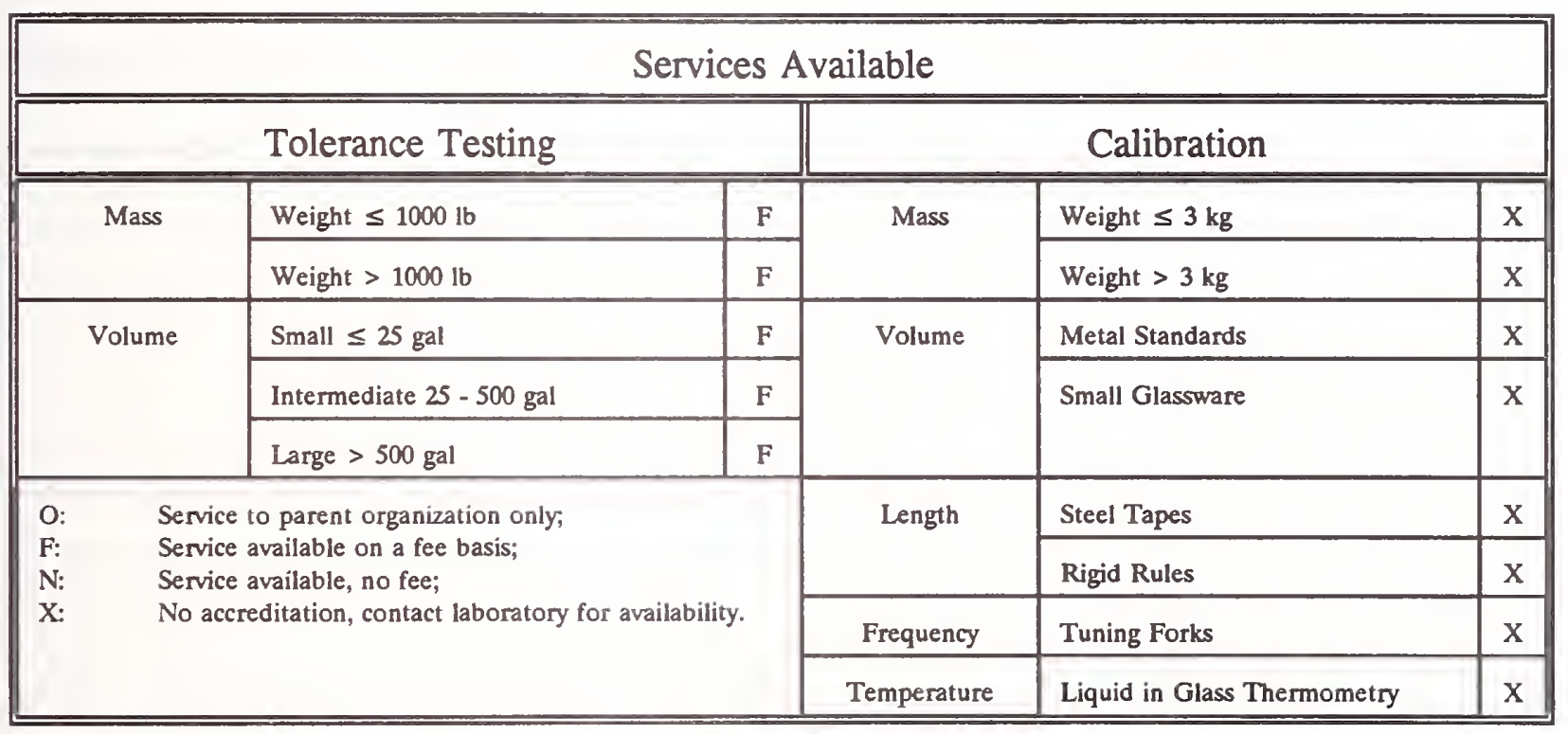

\begin{tabular}{|l||}
\hline Fees \\
\hline Contact laboratory for fee information. \\
\\
\hline
\end{tabular}




\section{Utah}

\begin{tabular}{|l|l||}
\hline \multicolumn{2}{|c|}{ Grain Moisture Testing } \\
\hline \hline Address & Telephone Number \\
\hline \hline & $(801)$ 392-2292 \\
\hline Person responsible for program & \\
\hline Contact for additional information & C. Larry Warner \\
\hline Additional accreditation/recognition & \\
\hline
\end{tabular}

\begin{tabular}{|l|l||}
\hline \multicolumn{2}{|c|}{ Petroleum Quality Testing } \\
\hline \hline Address & Telephone Number \\
\hline \hline $\begin{array}{l}\text { UT Department of Agriculture } \\
\text { Weights and Measures Division } \\
\text { 350 N Redwood Rd } \\
\text { Salt Lake City, UT 84116 }\end{array}$ & $(801)$ 538-7154 \\
\hline Person responsible for program & \\
\hline Contact for additional information & Monty Brown \\
\hline Additional accreditation/recognition & \\
\hline
\end{tabular}

\begin{tabular}{|l|l||}
\hline \multicolumn{2}{|c||}{ Additional Tests or Laboratory Functions } \\
\hline \hline Address & Telephone Number \\
\hline \hline & \\
\hline Person responsible for program & \\
\hline Contact for additional information & \\
\hline Additional accreditation/recognition & \\
\hline
\end{tabular}




\section{Vermont}

Accreditation Period: 1991-1993

\begin{tabular}{|l|l|l|}
\hline \hline Laboratory Staff & Address & Telephone Number \\
\hline \hline $\begin{array}{l}\text { Raymond P. Cioffi, Metrologist/Weights } \\
\text { and Measures Specialist }\end{array}$ & $\begin{array}{l}\text { Vermont Department of Agriculture } \\
\text { Labs } \\
\text { 103 S Main St } \\
\text { Waterbury, VT 05671-0101 }\end{array}$ & $\begin{array}{l}\text { (802) 244-4511 Lab } \\
\end{array}$ \\
& & \\
\hline
\end{tabular}

\begin{tabular}{|c|c|c|c|c|c|}
\hline \multicolumn{6}{|c|}{ Services Available } \\
\hline \multicolumn{3}{|c|}{ Tolerance Testing } & \multicolumn{3}{|c|}{ Calibration } \\
\hline \multirow[t]{2}{*}{ Mass } & Weight $\leq 1000 \mathrm{lb}$ & $\mathbf{F}$ & \multirow[t]{2}{*}{ Mass } & Weight $\leq 3 \mathrm{~kg}$ & $\mathbf{F}$ \\
\hline & Weight $>1000 \mathrm{lb}$ & $\mathrm{x}$ & & Weight $>3 \mathrm{~kg}$ & $\mathbf{F}$ \\
\hline \multirow[t]{3}{*}{ Volume } & Small $\leq 25$ gal & $\mathbf{F}$ & \multirow[t]{3}{*}{ Volume } & Metal Standards & $\mathbf{F}$ \\
\hline & Intermediate $25-500 \mathrm{gal}$ & $\mathbf{F}$ & & \multirow[t]{2}{*}{ Small Glassware } & \multirow[t]{2}{*}{$\mathbf{F}$} \\
\hline & Large $>500 \mathrm{gal}$ & $\mathrm{x}$ & & & \\
\hline \multirow{4}{*}{$\begin{array}{l}\text { O: } \\
\mathrm{F}: \\
\mathrm{N}: \\
\mathrm{X}:\end{array}$} & \multirow{4}{*}{\multicolumn{2}{|c|}{$\begin{array}{l}\text { Service to parent organization only; } \\
\text { Service available on a fee basis; } \\
\text { Service available, no fee; } \\
\text { No accreditation, contact laboratory for availability. }\end{array}$}} & \multirow[t]{2}{*}{ Length } & Steel Tapes & o \\
\hline & & & & Rigid Rules & $\mathrm{x}$ \\
\hline & & & Frequency & Tuning Forks & $\mathrm{X}$ \\
\hline & & & Temperature & Liquid in Glass Thermometry & $\mathrm{x}$ \\
\hline
\end{tabular}

\begin{tabular}{|l||}
\hline \multicolumn{1}{|c|}{ Fees } \\
\hline Labor...\$25 per hour. \\
Syrup hydrometers: $\$ 1.25$ \\
Sap hydrometers and certificate: $\$ 3$. \\
Refractometers: $\$ 25$ per hour. \\
\hline
\end{tabular}

\section{Remarks:}

Laboratory tests density hydrometers which are used in the syrup industry. Laboratory is not operated on a full-time basis. Wheel Load Weighers also tested $\$ 25.00 / \mathrm{hr}$. 


\section{Vermont}

\begin{tabular}{||l|l||}
\hline \multicolumn{2}{|c|}{ Grain Moisture Testing } \\
\hline \hline Address & Telephone Number \\
\hline \hline NOT AVAILABLE & \\
\hline Person responsible for program & \\
\hline Contact for additional information & \\
\hline Additional accreditation/recognition & \\
\hline
\end{tabular}

\begin{tabular}{||l|l||}
\hline \multicolumn{2}{|c|}{ Petroleum Quality Testing } \\
\hline \hline Address & Telephone Number \\
\hline Consumer Assurance Section \\
VT Department of Agriculture Food and Markets \\
120 State St \\
Montpelier, VT 05620-2901 & $(802) 828-2436$ \\
\hline Person responsible for program & \\
\hline Contact for additional information & Bruce Martell \\
\hline Additional accreditation/recognition & Ray Cioffi \\
\hline
\end{tabular}

\begin{tabular}{|l|l|}
\hline \multicolumn{2}{|c|}{ Additional Tests or Laboratory Functions } \\
\hline \hline Address & Telephone Number \\
\hline \hline $\begin{array}{l}\text { Vermont Department of Agriculture Labs } \\
\text { Wat Main St } \\
\text { Waterbury, VT 05671-0101 }\end{array}$ & $(802) 244-4510$ \\
\hline Person responsible for program & Bruce Martell \\
\hline Contact for additional information & Ray Cioffi \\
\hline Additional accreditation/recognition & Wheel Load Weigher Testing \\
\hline
\end{tabular}




\section{Virginia}

Accreditation Period: 1992-1994

\begin{tabular}{||l|l|l||}
\hline \hline Laboratory Staff & Address & Telephone Number \\
\hline \hline Michael J. Kramer, Inspector & $\begin{array}{l}\text { VA Dept of Agriculture and } \\
\text { Consumer Services } \\
\text { PO Box 1163 Rm 403 } \\
\text { Richmond, VA 23209 }\end{array}$ & FAX: (804) 371-0351 \\
\hline
\end{tabular}

\begin{tabular}{|c|c|c|c|c|c|}
\hline \multicolumn{6}{|c|}{ Services Available } \\
\hline \multicolumn{3}{|c|}{ Tolerance Testing } & \multicolumn{3}{|c|}{ Calibration } \\
\hline \multirow[t]{2}{*}{ Mass } & Weight $\leq 1000 \mathrm{lb}$ & $\mathrm{N}$ & \multirow[t]{2}{*}{ Mass } & Weight $\leq 3 \mathrm{~kg}$ & $\mathrm{~N}$ \\
\hline & Weight $>1000 \mathrm{lb}$ & $\mathrm{N}$ & & Weight $>3 \mathrm{~kg}$ & $\mathrm{~N}$ \\
\hline \multirow[t]{3}{*}{ Volume } & Small $\leq 25$ gal & $\mathrm{N}$ & \multirow[t]{3}{*}{ Volume } & Metal Standards & $\mathrm{N}$ \\
\hline & Intermediate $25-500 \mathrm{gal}$ & $\mathrm{N}$ & & \multirow[t]{2}{*}{ Small Glassware } & \multirow[t]{2}{*}{$\mathrm{N}$} \\
\hline & Large $>500$ gal & $\mathrm{N}$ & & & \\
\hline \multirow{4}{*}{$\begin{array}{l}\text { O: } \\
\text { F: } \\
\text { N: } \\
\text { X: }\end{array}$} & \multirow{4}{*}{\multicolumn{2}{|c|}{$\begin{array}{l}\text { Service to parent organization only; } \\
\text { Service available on a fee basis; } \\
\text { Service available, no fee; } \\
\text { No accreditation, contact laboratory for availability. }\end{array}$}} & \multirow[t]{2}{*}{ Length } & Steel Tapes & $\mathrm{N}$ \\
\hline & & & & Rigid Rules & $\mathrm{N}$ \\
\hline & & & Frequency & Tuning Forks & $\mathrm{N}$ \\
\hline & & & Temperature & Liquid in Glass Thermometry & $\mathrm{N}$ \\
\hline
\end{tabular}

\section{Fees}

No fees are charged.

\section{Remarks:}

Laboratory also tests Babcock dairy bottles and other associated glassware. 


\section{Virginia}

\begin{tabular}{|l|l||}
\hline \multicolumn{2}{|c|}{ Grain Moisture Testing } \\
\hline \hline Address & Telephone Number \\
\hline \hline $\begin{array}{l}\text { VA Department of Agriculture and Consumer Services } \\
\text { PO Box 1163 } \\
\text { Richmond, VA 23209 }\end{array}$ & $(804)$ 786-3939 \\
\hline Person responsible for program & W. Randy Sanford \\
\hline Contact for additional information & \\
\hline Additional accreditation/recognition & \\
\hline
\end{tabular}

\begin{tabular}{|l|l||}
\hline \multicolumn{2}{|c|}{ Petroleum Quality Testing } \\
\hline \hline Address & Telephone Number \\
\hline \hline $\begin{array}{l}\text { VA Department of Agriculture and Consumer Services } \\
\text { PO Box 1163 } \\
\text { Richmond, VA 23209 }\end{array}$ & \\
\hline Person responsible for program $786-2476$ \\
\hline Contact for additional information & Supervisory Weights and Measures Staff \\
\hline Additional accreditation/recognition & \\
\hline
\end{tabular}

\begin{tabular}{||l|l||}
\hline \multicolumn{2}{|c||}{ Additional Tests or Laboratory Functions } \\
\hline \hline Address & Telephone Number \\
\hline & \\
\hline Person responsible for program & \\
\hline Contact for additional information & \\
\hline Additional accreditation/recognition & \\
\hline \hline
\end{tabular}




\section{Virgin Islands}

Not Currentlly Accredited

\begin{tabular}{||l|l|l||}
\hline \hline Laboratory Staff & Address & Telephone Number \\
\hline \hline Sandra Croft, Metrologist & $\begin{array}{l}\text { Consumer Service Division } \\
\text { Golden Rock Shopping Center } \\
\text { Christiansted St VI 00820 }\end{array}$ & FAX: (809) 778-8250 \\
\hline
\end{tabular}

\begin{tabular}{|c|c|c|c|c|c|}
\hline \multicolumn{6}{|c|}{ Services Available } \\
\hline \multicolumn{3}{|c|}{ Tolerance Testing } & \multicolumn{3}{|c|}{ Calibration } \\
\hline \multirow[t]{2}{*}{ Mass } & Weight $\leq 1000 \mathrm{lb}$ & $\mathrm{x}$ & \multirow[t]{2}{*}{ Mass } & Weight $\leq 3 \mathrm{~kg}$ & $\mathrm{x}$ \\
\hline & Weight $>1000 \mathrm{lb}$ & $\mathrm{x}$ & & Weight $>3 \mathrm{~kg}$ & $\mathrm{x}$ \\
\hline \multirow[t]{3}{*}{ Volume } & Small $\leq 25$ gal & $\mathrm{x}$ & \multirow[t]{3}{*}{ Volume } & Metal Standards & $\mathrm{x}$ \\
\hline & Intermediate 25 - $500 \mathrm{gal}$ & $\mathrm{x}$ & & \multirow[t]{2}{*}{ Small Glassware } & \multirow[t]{2}{*}{$\mathrm{x}$} \\
\hline & Large > 500 gal & $\mathrm{x}$ & & & \\
\hline \multirow{4}{*}{$\begin{array}{l}\text { O: } \\
\text { F: } \\
\text { N: } \\
X:\end{array}$} & \multirow{4}{*}{\multicolumn{2}{|c|}{$\begin{array}{l}\text { Service to parent organization only; } \\
\text { Service available on a fee basis; } \\
\text { Service available, no fee; } \\
\text { No accreditation, contact laboratory for availability. }\end{array}$}} & \multirow[t]{2}{*}{ Length } & Steel Tapes & $\mathrm{x}$ \\
\hline & & & & Rigid Rules & $\mathrm{x}$ \\
\hline & & & Frequency & Tuning Forks & $\mathrm{x}$ \\
\hline & & & Temperature & Liquid in Glass Thermometry & $\mathrm{x}$ \\
\hline
\end{tabular}

\begin{tabular}{|c||}
\hline Fees \\
\hline \hline \\
\hline
\end{tabular}

Remarks:

Laboratory equipment and standards were destroyed by Hurricane Hugo in 1989. 


\section{Virgin Islands}

\begin{tabular}{||l|l||}
\hline \multicolumn{2}{|c||}{ Grain Moisture Testing } \\
\hline \hline Address & Telephone Number \\
\hline \hline NOT AVAILABLE & \\
\hline Person responsible for program & \\
\hline Contact for additional information & \\
\hline Additional accreditation/recognition & \\
\hline
\end{tabular}

\begin{tabular}{|l|l||}
\hline \multicolumn{2}{|c|}{ Petroleum Quality Testing } \\
\hline \hline Address & Telephone Number \\
\hline \hline NOT AVAILABLE & \\
& \\
\hline Person responsible for program & \\
\hline Contact for additional information & \\
\hline Additional accreditation/recognition & \\
\hline \hline
\end{tabular}

\begin{tabular}{||l|l||}
\hline \multicolumn{2}{|c||}{ Additional Tests or Laboratory Functions } \\
\hline \hline Address & Telephone Number \\
\hline \hline & \\
\hline Person responsible for program & \\
\hline Contact for additional information & \\
\hline Additional accreditation/recognition & \\
\hline \hline
\end{tabular}




\section{Washington}

\section{Accreditation Period: 1991-1993}

\begin{tabular}{||l||l|l||}
\hline \hline Laboratory Staff & Address & Telephone Number \\
\hline \hline James H. Cammel, Metrologist, Inspector III & & (206) 753-5042 \\
John W. Allen, Asst Metrologist & $\begin{array}{l}\text { Dept of Agriculture } \\
\text { Weights and Measures Section } \\
\text { PO Box 42560 } \\
\text { Olympia, WA 98504-2560 }\end{array}$ & \\
& & \\
& & \\
\hline
\end{tabular}

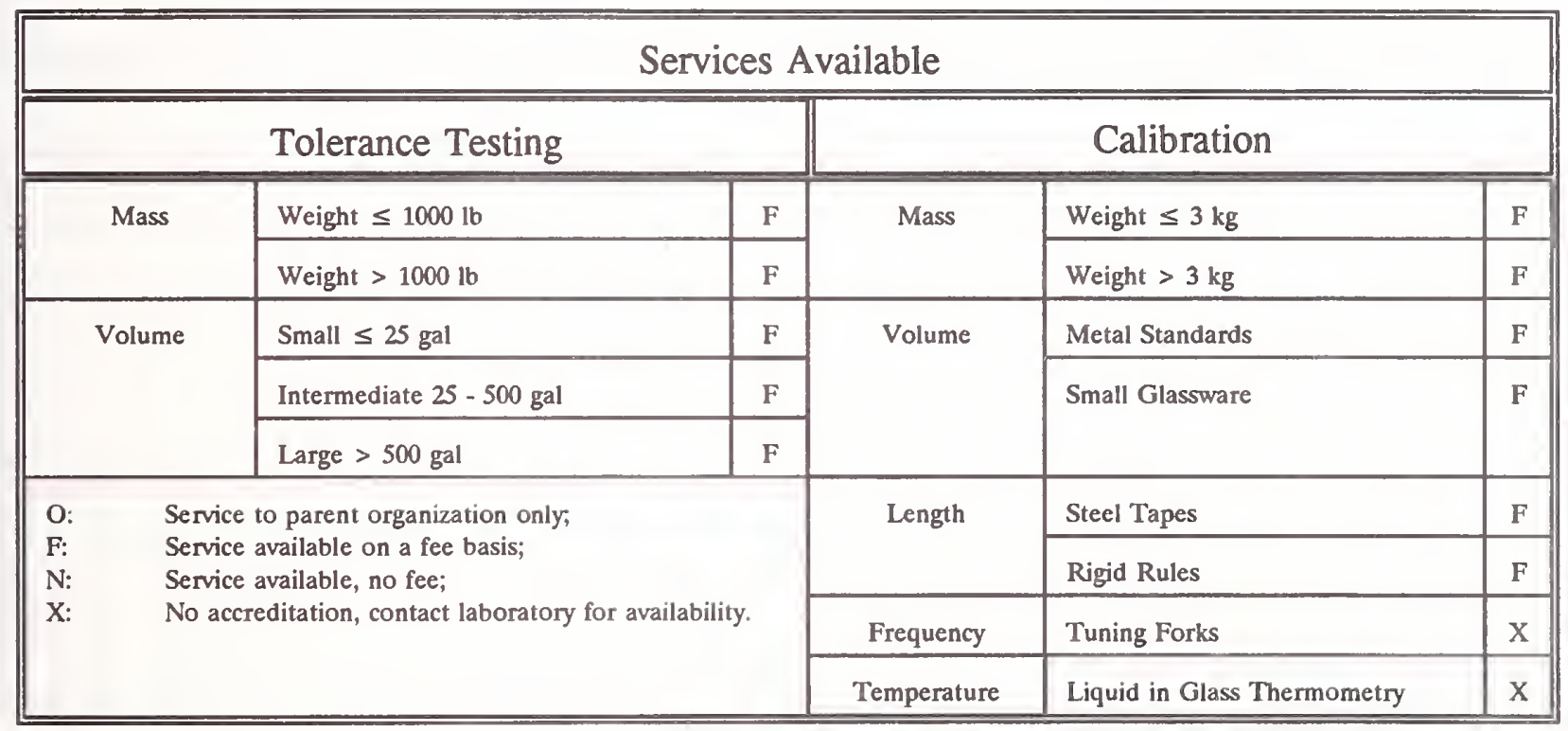

\section{Fees}

Fees vary according to type of artifact and type of service requested. Contact laboratory directly for specific fee information.

Repairs, if necessary, will be made only with owner-approval at the rate of $\$ 25$ per hour. 


\section{Washington}

\begin{tabular}{||l|l||}
\hline \multicolumn{2}{|c||}{ Grain Moisture Testing } \\
\hline \hline Address & Telephone Number \\
\hline \hline NOT AVAILABLE & \\
\hline Person responsible for program & \\
\hline Contact for additional information & \\
\hline Additional accreditation/recognition & \\
\hline
\end{tabular}

\begin{tabular}{||l|l||}
\hline \multicolumn{2}{|c||}{ Petroleum Quality Testing } \\
\hline \hline Address & Telephone Number \\
\hline \hline NOT AVAILABLE & \\
& \\
\hline Person responsible for program & \\
\hline Contact for additional information & \\
\hline Additional accreditation/recognition & \\
\hline
\end{tabular}

\begin{tabular}{||l|l||}
\hline \multicolumn{2}{|c||}{ Additional Tests or Laboratory Functions } \\
\hline \hline Address & Telephone Number \\
\hline & \\
\hline Person responsible for program & \\
\hline Contact for additional information & \\
\hline Additional accreditation/recognition & \\
\hline
\end{tabular}




\section{West Virginia}

Not Currently Accredited

\begin{tabular}{|l|l||l||}
\hline \hline Laboratory Staff & Address & Telephone Number \\
\hline Dannie Abshire, Inspector II & & (304) 722-0602 \\
Dave Hopkins & $\begin{array}{l}\text { WV Dept of Labor } \\
\text { Weights and Measures Division } \\
\text { 570 MacCorkle Ave } \\
\text { St. Albans, WV 25117 }\end{array}$ & FAX: (304) 348-3797 \\
& & \\
\hline
\end{tabular}

\begin{tabular}{|c|c|c|c|c|c|}
\hline \multicolumn{6}{|c|}{ Services Available } \\
\hline \multicolumn{3}{|c|}{ Tolerance Testing } & \multicolumn{3}{|c|}{ Calibration } \\
\hline \multirow[t]{2}{*}{ Mass } & Weight $\leq 1000 \mathrm{lb}$ & $\mathbf{N}$ & \multirow[t]{2}{*}{ Mass } & Weight $\leq 3 \mathrm{~kg}$ & $\mathrm{~N}$ \\
\hline & Weight $>1000 \mathrm{lb}$ & $\mathrm{N}$ & & Weight $>3 \mathrm{~kg}$ & $\mathrm{~N}$ \\
\hline \multirow[t]{3}{*}{ Volume } & Small $\leq 25$ gal & $\mathrm{N}$ & \multirow[t]{3}{*}{ Volume } & Metal Standards & $\mathrm{N}$ \\
\hline & Intermediate $25-500$ gal & $\mathrm{N}$ & & \multirow[t]{2}{*}{ Small Glassware } & \multirow[t]{2}{*}{$\mathrm{N}$} \\
\hline & Large > $500 \mathrm{gal}$ & $\mathrm{N}$ & & & \\
\hline \multirow{4}{*}{$\begin{array}{l}\text { O: } \\
\text { F: } \\
\text { N: } \\
\text { X: }\end{array}$} & \multirow{4}{*}{\multicolumn{2}{|c|}{$\begin{array}{l}\text { Service to parent organization only; } \\
\text { Service available on a fee basis; } \\
\text { Service available, no fee; } \\
\text { No accreditation, contact laboratory for availability. }\end{array}$}} & \multirow[t]{2}{*}{ Length } & Steel Tapes & $\mathrm{N}$ \\
\hline & & & & Rigid Rules & $\mathrm{N}$ \\
\hline & & & Frequency & Tuning Forks & $\mathrm{x}$ \\
\hline & & & Temperature & Liquid in Glass Thermometry & $\mathrm{x}$ \\
\hline
\end{tabular}

\section{Fees}

No fees are charged. 


\section{West Virginia}

\begin{tabular}{||l|l||}
\hline \multicolumn{2}{|c||}{ Grain Moisture Testing } \\
\hline \hline Address & Telephone Number \\
\hline \hline NOT AVAILABLE & \\
\hline Person responsible for program & \\
\hline Contact for additional information & \\
\hline Additional accreditation/recognition & \\
\hline
\end{tabular}

\begin{tabular}{||l|l||}
\hline \multicolumn{2}{|c||}{ Petroleum Quality Testing } \\
\hline \hline Address & Telephone Number \\
\hline \hline NOT AVAILABLE & \\
\hline Person responsible for program & \\
\hline Contact for additional information & \\
\hline Additional accreditation/recognition & \\
\hline
\end{tabular}

\begin{tabular}{|l|l||}
\hline \multicolumn{2}{|c||}{ Additional Tests or Laboratory Functions } \\
\hline \hline Address & Telephone Number \\
\hline \hline & \\
\hline Person responsible for program & \\
\hline Contact for additional information & \\
\hline Additional accreditation/recognition & \\
\hline
\end{tabular}




\section{Wisconsin}

Accreditation Period: 1991-1993

\begin{tabular}{|l|l|l||}
\hline \hline Laboratory Staff & Address & Telephone Number \\
\hline James H. Akey, Metrologist & $\begin{array}{l}\text { WI Dept of Agriculture } \\
\text { Trade and Consumer Protection } \\
\text { 4702 University Ave } \\
\text { Madison, WI 53705 }\end{array}$ & $\begin{array}{l}\text { (608) 267-3510 Lab } \\
\text { Forter, Technical Supervisor }\end{array}$ \\
& $\begin{array}{l}\text { Po Box 7883 (608) 266-1560 } \\
\text { Madison, WI 53707-7883 }\end{array}$ & \\
\hline
\end{tabular}

\begin{tabular}{|c|c|c|c|c|c|}
\hline \multicolumn{6}{|c|}{ Services Available } \\
\hline \multicolumn{3}{|c|}{ Tolerance Testing } & \multicolumn{3}{|c|}{ Calibration } \\
\hline \multirow[t]{2}{*}{ Mass } & Weight $\leq 1000 \mathrm{lb}$ & $\mathbf{F}$ & \multirow[t]{2}{*}{ Mass } & Weight $\leq 3 \mathrm{~kg}$ & $\mathbf{F}$ \\
\hline & Weight $>1000 \mathrm{lb}$ up to $2500 \mathrm{lb}$ & $\mathrm{F}$ & & Weight $>3 \mathrm{~kg}$ & $\mathrm{~F}$ \\
\hline \multirow[t]{3}{*}{ Volume } & Small $\leq 25$ gal & $\mathbf{F}$ & \multirow[t]{3}{*}{ Volume } & Metal Standards & $\mathbf{F}$ \\
\hline & Intermediate $25-500 \mathrm{gal}$ & $\mathrm{F}$ & & \multirow[t]{2}{*}{ Small Glassware } & \multirow[t]{2}{*}{$\mathbf{F}$} \\
\hline & Large $>500$ gal & $\mathrm{F}$ & & & \\
\hline \multirow{4}{*}{$\begin{array}{l}\text { O: } \\
\text { F: } \\
\text { N: } \\
\text { X: }\end{array}$} & \multirow{4}{*}{\multicolumn{2}{|c|}{$\begin{array}{l}\text { Service to parent organization only; } \\
\text { Service available on a fee basis; } \\
\text { Service available, no fee; } \\
\text { No accreditation, contact laboratory for availability. }\end{array}$}} & \multirow[t]{2}{*}{ Length } & Steel Tapes & $\mathbf{F}$ \\
\hline & & & & Rigid Rules & $\mathrm{F}$ \\
\hline & & & Frequency & Tuning Forks & $\mathrm{x}$ \\
\hline & & & Temperature & Liquid in Glass Thermometry & $\mathrm{x}$ \\
\hline
\end{tabular}

\section{Fees}

Labor... $\$ 50$ per hour. Minimum charge is $1 / 2$ hour.

Verification of weights and measures used in enforcement work by State, county, and municipal governments is exempt from fees.

Mass, tolerance testing: $500 \mathrm{lb}, 1,000 \mathrm{lb}$ : $\$ 12.50$ each; all smaller weights $\$ 6.00$ each. Weight sets (<25 pes): $\$ 50$. Adjusted weights charged double fees.

Volume: glass flasks: $\$ 50.00$; field test measures, 1 and 5 gal: $\$ 15.00$; provers, 50 and 100 gal: $\$ 50.00$. $>100$ gal at hourly rate.

\section{Remarks:}

The Wisconsin laboratory also provides vapor meter testing with a bell jar prover. 


\section{Wisconsin}

\begin{tabular}{||l|l||}
\hline \multicolumn{2}{|c|}{ Grain Moisture Testing } \\
\hline \hline Address & Telephone Number \\
\hline \hline PO Box 7883 \\
Madison, WI 53707-7883 & $(608)$ 267-3510 \\
\hline Person responsible for program & \\
\hline Contact for additional information & James H. Akey \\
\hline Additional accreditation/recognition & \\
\hline
\end{tabular}

\begin{tabular}{||l|l||}
\hline \multicolumn{2}{|c||}{ Petroleum Quality Testing } \\
\hline \hline Address & Telephone Number \\
\hline \hline $\begin{array}{l}\text { Department of Industry, Labor and Human Relations } \\
\text { Petroleum Inspection } \\
201 \text { E Washington Ave } \\
\text { Madison, WI 53703 }\end{array}$ & (608) 266-8076 \\
\hline Person responsible for program & \\
\hline Contact for additional information & \\
\hline Additional accreditation/recognition & \\
\hline
\end{tabular}

\begin{tabular}{||l|l||}
\hline \multicolumn{2}{|c|}{ Additional Tests or Laboratory Functions } \\
\hline \hline Address & Telephone Number \\
\hline & \\
\hline Person responsible for program & \\
\hline Contact for additional information & \\
\hline Additional accreditation/recognition & \\
\hline
\end{tabular}




\section{Wyoming}

Accreditation Period: 1993

\begin{tabular}{||l|l|l||}
\hline \hline Laboratory Staff & Address & Telephone Number \\
\hline \hline Victor Gerber, Metrologist & $\begin{array}{l}\text { WY Dept of Agriculture } \\
\text { 2219 Carey Ave } \\
\text { Cheyenne, WY 82002 }\end{array}$ & FAX: (307) 777-6593 \\
& & \\
\hline
\end{tabular}

\begin{tabular}{|c|c|c|c|c|c|}
\hline \multicolumn{6}{|c|}{ Services Available } \\
\hline \multicolumn{3}{|c|}{ Tolerance Testing } & \multicolumn{3}{|c|}{ Calibration } \\
\hline \multirow[t]{2}{*}{ Mass } & Weight $\leq 1000 \mathrm{lb}$ & $\mathrm{F}$ & \multirow[t]{2}{*}{ Mass } & Weight $\leq 3 \mathrm{~kg}$ & $\mathrm{x}$ \\
\hline & Weight $>1000 \mathrm{lb}$ & $\mathrm{F}$ & & Weight $>3 \mathrm{~kg}$ & $\mathrm{x}$ \\
\hline \multirow[t]{3}{*}{ Volume } & Small $\leq 25$ gal & $\mathrm{F}$ & \multirow[t]{3}{*}{ Volume } & Metal Standards & $\mathrm{x}$ \\
\hline & Intermediate $25-500 \mathrm{gal}$ & $\mathrm{F}$ & & \multirow[t]{2}{*}{ Small Glassware } & \multirow[t]{2}{*}{$\mathrm{x}$} \\
\hline & Large $>500 \mathrm{gal}$ & o & & & \\
\hline \multirow{4}{*}{$\begin{array}{l}\text { O: } \\
\mathrm{F}: \\
\mathrm{N}: \\
\mathrm{X}:\end{array}$} & \multirow{4}{*}{\multicolumn{2}{|c|}{$\begin{array}{l}\text { Service to parent organization only; } \\
\text { Service available on a fee basis; } \\
\text { Service available, no fee; } \\
\text { No accreditation, contact laboratory for availability. }\end{array}$}} & \multirow[t]{2}{*}{ Length } & Steel Tapes & $\mathrm{x}$ \\
\hline & & & & Rigid Rules & $\mathrm{x}$ \\
\hline & & & Frequency & Tuning Forks & $\mathrm{N}$ \\
\hline & & & Temperature & Liquid in Glass Thermometry & $\mathrm{x}$ \\
\hline
\end{tabular}

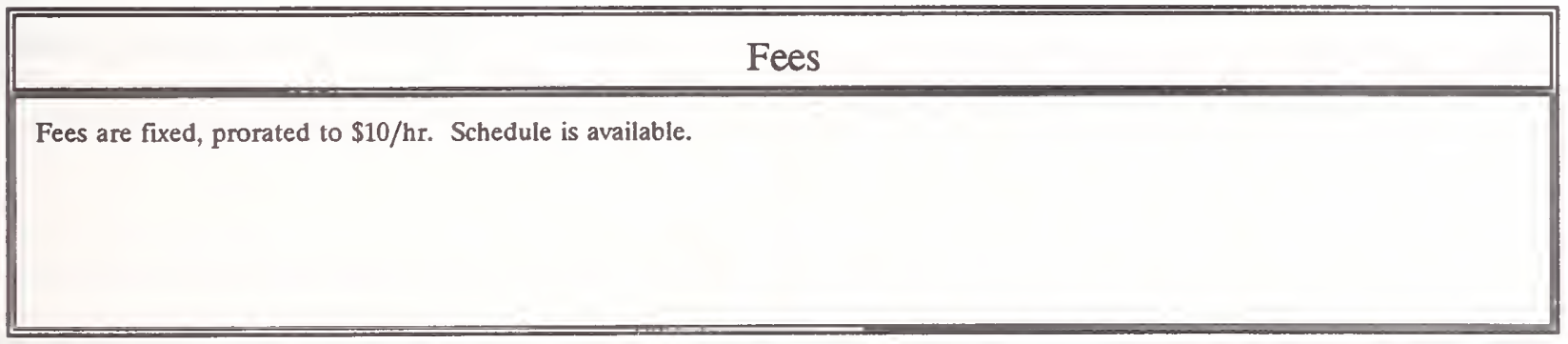

\section{Remarks:}

Wyoming moved into a new laboratory facility in 1988. 


\section{Wyoming}

\begin{tabular}{|l|l||}
\hline \multicolumn{2}{|c|}{ Grain Moisture Testing } \\
\hline \hline Address & Telephone Number \\
\hline \hline NOT AVAILABLE & \\
\hline Person responsible for program & \\
\hline Contact for additional information & \\
\hline Additional accreditation/recognition & \\
\hline
\end{tabular}

\begin{tabular}{||l|l||}
\hline \multicolumn{2}{|c|}{ Petroleum Quality Testing } \\
\hline \hline Address & Telephone Number \\
\hline \hline $\begin{array}{l}\text { Chemistry and Bacteriological Laboratory } \\
\text { 1174 Snowy Range Rd. } \\
\text { Laramie, WY 82070 }\end{array}$ & $\begin{array}{l}(307) 742-2984 \\
\text { FAX: (307) 742-2156 }\end{array}$ \\
\hline Person responsible for program & Ken Mcmillan, State Chemist \\
\hline Contact for additional information & \\
\hline Additional accreditation/recognition & \\
\hline
\end{tabular}

\begin{tabular}{||l|l||}
\hline \multicolumn{2}{|c||}{ Additional Tests or Laboratory Functions } \\
\hline \hline Address & Telephone Number \\
\hline \hline & \\
& \\
\hline Person responsible for program & \\
\hline Contact for additional information & \\
\hline Additional accreditation/recognition & \\
\hline
\end{tabular}




\section{USDA/FGIS}

Accreditation Period: 1992-1994

\begin{tabular}{||l|l||l||}
\hline \hline Laboratory Staff & Address & Telephone Number \\
\hline \hline Paul Hadyka, Industrial Specialist & USDA/FGIS & (202) 720-0262 (DC) \\
Willis Norrs, Industrial Specialist & $\begin{array}{l}\text { Room 1640-S PO Box 96454 } \\
\text { 1400 Independence Ave SW } \\
\text { Fred Anderson, Industrial Specialist }\end{array}$ & FAX: (202) 720-1015 \\
& $\begin{array}{l}\text { Waster Scale Depot } \\
5800 \text { W. 69th St } \\
\text { Chicago, IL 60638 }\end{array}$ & (708) 458-0655 (IL) \\
& & \\
\hline
\end{tabular}

\begin{tabular}{|c|c|c|c|c|c|}
\hline \multicolumn{6}{|c|}{ Services Available } \\
\hline \multicolumn{3}{|c|}{ Tolerance Testing } & \multicolumn{3}{|c|}{ Calibration } \\
\hline \multirow[t]{2}{*}{ Mass } & Weight $\leq 1000 \mathrm{lb}$ & $\mathrm{F}$ & \multirow[t]{2}{*}{ Mass } & Weight $\leq 3 \mathrm{~kg}$ & $\mathrm{X}$ \\
\hline & Weight $>1000 \mathrm{lb}$ up to $10,000 \mathrm{lb}$ & $\mathrm{F}$ & & Weight $>3 \mathrm{~kg}$ up to $10,000 \mathrm{lb}$ & $\mathrm{F}$ \\
\hline \multirow[t]{3}{*}{ Volume } & Small $\leq 25$ gal & $\mathrm{X}$ & \multirow[t]{3}{*}{ Volume } & Metal Standards & $\mathrm{X}$ \\
\hline & Intermediate 25 - $500 \mathrm{gal}$ & $\mathrm{x}$ & & \multirow[t]{2}{*}{ Small Glassware } & \multirow[t]{2}{*}{$\mathrm{x}$} \\
\hline & Large > $500 \mathrm{gal}$ & $\mathrm{x}$ & & & \\
\hline \multirow{4}{*}{$\begin{array}{l}\text { O: } \\
\text { F: } \\
\mathrm{N}: \\
\mathrm{X}:\end{array}$} & \multirow{4}{*}{\multicolumn{2}{|c|}{$\begin{array}{l}\text { Service to parent organization only; } \\
\text { Service available on a fee basis; } \\
\text { Service available, no fee; } \\
\text { No accreditation, contact laboratory for availability. }\end{array}$}} & \multirow[t]{2}{*}{ Length } & Steel Tapes & $\mathrm{X}$ \\
\hline & & & & Rigid Rules & $\mathrm{X}$ \\
\hline & & & Frequency & Tuning Forks & $\mathrm{X}$ \\
\hline & & & Temperature & Liquid in Glass Thermometry & $\mathrm{x}$ \\
\hline
\end{tabular}

\begin{tabular}{||l||}
\hline Fees \\
\hline \hline Labor...\$41.90 per hour. \\
\\
\hline
\end{tabular}

\section{Remarks:}

Tolerance testing of $50 \mathrm{lb}$ test weights and mass standards from $500 \mathrm{lb}$ to $10,000 \mathrm{lb}$ is provided by at: USDA, FGIS, Master Scale Depot, 5800 W. 69 th St, Chicago, IL 60638. Also calibration of 2,500 to $10,000 \mathrm{lb}$ at Master Scale. 


\section{USDA/FGIS}

\begin{tabular}{||l|l||}
\hline \multicolumn{2}{|c|}{ Grain Moisture Testing } \\
\hline \hline Address & Telephone Number \\
\hline $\begin{array}{l}\text { USDA FGIS Standardization QC Branch } \\
\text { PO Box 20285 } \\
\text { Kansas City, MO 64195 }\end{array}$ & $\begin{array}{l}\text { (816) 891-8070 } \\
\text { (816) 374-6547 }\end{array}$ \\
\hline Person responsible for program & James Rampton \\
\hline Contact for additional information & Ron Bicak \\
\hline Additional accreditation/recognition & \\
\hline
\end{tabular}

\begin{tabular}{|l|l||}
\hline \multicolumn{2}{|c||}{ National Type Evaluation Program (NTEP) } \\
\hline \hline Address & Telephone Number \\
\hline $\begin{array}{l}\text { USDA, FGIS Room 1640-S } \\
\text { PO Box 96454 } \\
\text { Washington, D.C. 20090-6454 }\end{array}$ & (202) 720-0262 \\
\hline Person responsible for program & Richard Pforr \\
\hline Contact for additional information & \\
\hline Additional accreditation/recognition & NIST Authorized Participating Laboratory \\
\hline NTEP testing of automatic bulk weighing systems, railway track scales, and grain moisture scales. \\
\hline
\end{tabular}

\begin{tabular}{|l|l||}
\hline \multicolumn{2}{|c||}{ Additional Tests or Laboratory Functions } \\
\hline \hline Address & Telephone Number \\
\hline & \\
\hline Person responsible for program & \\
\hline Contact for additional information & \\
\hline Additional accreditation/recognition & \\
\hline \hline
\end{tabular}




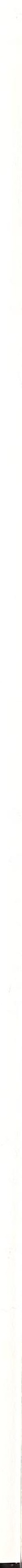


Journal of Research of the National Institute of Standards and Technology-Reports NIST research and development in those disciplines of the physical and engineering sciences in which the Institute is active. These include physics, chemistry, engineering, mathematics, and computer sciences. Papers cover a broad range of subjects, with major emphasis on measurement methodology and the basic technology underlying standardization. Also included from time to time are survey articles on topics closely related to the Institute's technical and scientific programs. Issued six times a year.

\section{Nonperiodicals}

Monographs-Major contributions to the technical literature on various subjects related to the Institute's scientific and technical activities.

Handbooks-Recommended codes of engineering and industrial practice (including safety codes) developed in cooperation with interested industries, professional organizations, and regulatory bodies.

Special Publications - Include proceedings of conferences sponsored by NIST, NIST annual reports, and other special publications appropriate to this grouping such as wall charts, pocket cards, and bibliographies.

Applied Mathematics Series-Mathematical tables, manuals, and studies of special interest to physicists, engineers, chemists, biologists, mathematicians, computer programmers, and others engaged in scientific and technical work.

National Standard Reference Data Series-Provides quantitative data on the physical and chemical properties of materials, compiled from the world's literature and critically evaluated. Developed under a worldwide program coordinated by NIST under the authority of the National Standard Data Act (Public Law 90-396). NOTE: The Journal of Physical and Chemical Reference Data (JPCRD) is published bimonthly for NIST by the American Chemical Society (ACS) and the American Institute of Physics (AIP). Subscriptions, reprints, and supplements are available from ACS, 1155 Sixteenth St., NW, Washington, DC 20056.

Building Science Series-Disseminates technical information developed at the Institute on building materials, components, systems, and whole structures. The series presents research results, test methods, and performance criteria related to the structural and environmental functions and the durability and safety characteristics of building elements and systems.

Technical Notes-Studies or reports which are complete in themselves but restrictive in their treatment of a subject. Analogous to monographs but not so comprehensive in scope or definitive in treatment of the subject area. Often serve as a vehicle for final reports of work performed at NIST under the sponsorship of other government agencies.

Voluntary Product Standards - Developed under procedures published by the Department of Commerce in Part 10, Title 15, of the Code of Federal Regulations. The standards establish nationally recognized requirements for products, and provide all concerned interests with a basis for common understanding of the characteristics of the products. NIST administers this program in support of the efforts of private-sector standardizing organizations.

Consumer Information Series - Practical information, based on NIST research and experience, covering areas of interest to the consumer. Easily understandable language and illustrations provide useful background knowledge for shopping in today's technological marketplace.

Order the above NIST publications from: Superintendent of Documents, Government Printing Office, Washington, DC 20402.

Order the following NIST publications-FIPS and NISTIRs-from the National Technical Information Service, Springfield, VA 22161.

Federal Information Processing Standards Publications (FIPS PUB)-Publications in this series collectively constitute the Federal Information Processing Standards Register. The Register serves as the official source of information in the Federal Government regarding standards issued by NIST pursuant to the Federal Property and Administrative Services Act of 1949 as amended, Public Law 89-306 (79 Stat. 1127), and as implemented by Executive Order 11717 (38 FR 12315, dated May 11, 1973) and Part 6 of Title 15 CFR (Code of Federal Regulations).

NIST Interagency Reports (NISTIR)-A special series of interim or final reports on work performed by NIST for outside sponsors (both government and non-government). In general, initial distribution is handled by the sponsor; public distribution is by the National Technical Information Service, Springfield, VA 22161, in paper copy or microfiche form. 

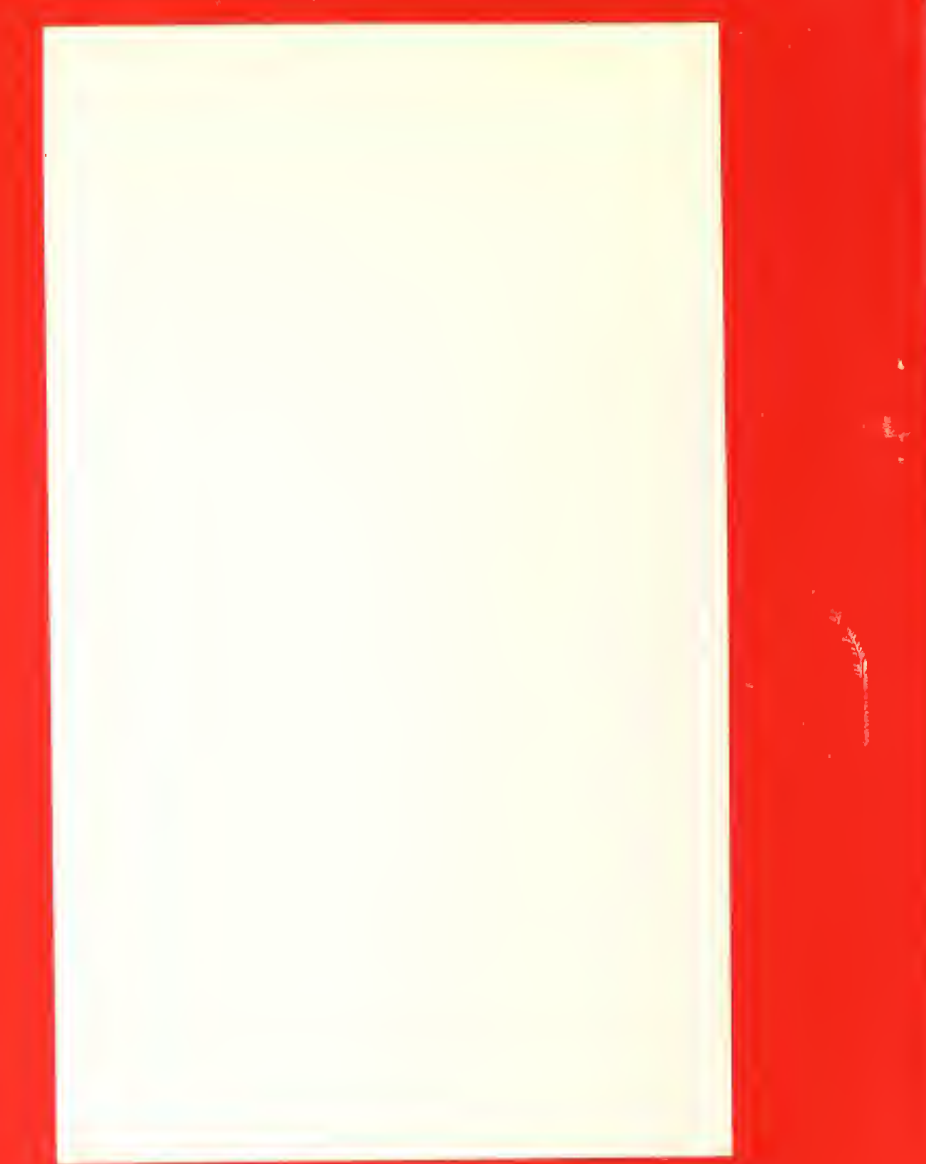

흥

8 ํㅜㄹ

낭용

를

8.

ऊू.

ยทก :

우워

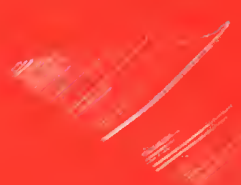

